\title{
Diagnostics of irradiated dense gas in galaxy nuclei
}

\section{A grid of XDR and PDR models ${ }^{\star}$}

\author{
R. Meijerink ${ }^{1}$, M. Spaans ${ }^{2}$, and F. P. Israel ${ }^{1}$ \\ 1 Sterrewacht Leiden, PO Box 9513, 2300 RA, Leiden, The Netherlands \\ e-mail: rowin@astro.berkeley.edu \\ 2 Kapteyn Astronomical Institute, PO Box 800, 9700 AV Groningen, The Netherlands
}

Received 28 July 2006 / Accepted 6 October 2006

ABSTRACT

\begin{abstract}
Aims. The nuclei of active galaxies harbor massive young stars, an accreting central black hole, or both. In order to determine the physical conditions that pertain to molecular gas close to the sources of radiation, numerical models are constructed. Methods. These models iteratively determine the thermal and chemical balance of molecular gas that is exposed to X-rays (1-100 keV) and far-ultraviolet radiation $(6-13.6 \mathrm{eV})$, as a function of depth.

Results. We present a grid of XDR and PDR models that span ranges in density $\left(10^{2}-10^{6.5} \mathrm{~cm}^{-3}\right)$, irradiation $\left(10^{0.5}-10^{5} G_{0}\right.$ and $\left.F_{\mathrm{X}}=1.6 \times 10^{-2}-160 \mathrm{erg} \mathrm{cm}^{-2} \mathrm{~s}^{-1}\right)$ and column density $\left(3 \times 10^{21}-1 \times 10^{25} \mathrm{~cm}^{-2}\right)$. Predictions are made for the most important atomic fine-structure lines, e.g., [CII], [OI], [CI], [SiII], and for molecular species like $\mathrm{HCO}^{+}, \mathrm{HCN}, \mathrm{HNC}, \mathrm{CS}$ and $\mathrm{SiO}$ up to $J=4, \mathrm{CO}$ and ${ }^{13} \mathrm{CO}$ up to $J=16$, and column densities for $\mathrm{CN}, \mathrm{CH}, \mathrm{CH}^{+}, \mathrm{HCO}, \mathrm{HOC}^{+}, \mathrm{NO}$ and $\mathrm{N}_{2} \mathrm{H}^{+}$. We find that surface temperatures are higher (lower) in PDRs compared to XDRs for densities $>10^{4}\left(<10^{4}\right) \mathrm{cm}^{-3}$. For the atomic lines, we find that, largely due to the different XDR ionization balance, the fine-structure line ratios of [SiII] $35 \mu \mathrm{m} /[\mathrm{CII}] 158 \mu \mathrm{m}$, [OI] $63 \mu \mathrm{m} /[\mathrm{CII}] 158 \mu \mathrm{m}$, [FeII] $26 \mu \mathrm{m} /[\mathrm{CII}] 158 \mu \mathrm{m}$ and [CI] $369 \mu \mathrm{m} /[\mathrm{CI}] 609 \mu \mathrm{m}$ are larger in XDRs than in PDRs, for a given density, column and irradiation strength. Similarly, for the molecular lines, we find that the line ratios $\mathrm{HCN} / \mathrm{HCO}^{+}$and $\mathrm{HNC} / \mathrm{HCN}$, as well as the column density ratio $\mathrm{CN} / \mathrm{HCN}$, discriminate between PDRs and XDRs. In particular, the $\mathrm{HCN} / \mathrm{HCO}^{+} 1-0$ ratio is $<1$ ( $>1$ ) for XDRs (PDRs) if the density exceeds $10^{5} \mathrm{~cm}^{-3}$ and if the column density is larger than $10^{23} \mathrm{~cm}^{-2}$. For columns less than $10^{22.5} \mathrm{~cm}^{-2}$ the XDR HCN/HCO 1-0 ratio becomes larger than one, although the individual $\mathrm{HCN} 1-0$ and $\mathrm{HCO}^{+} 1-0$ line intensities are weaker. For modest densities, $n=10^{4}-10^{5} \mathrm{~cm}^{-3}$, and strong radiation fields $\left(>100 \mathrm{erg} \mathrm{s}^{-1} \mathrm{~cm}^{-2}\right), \mathrm{HCN} / \mathrm{HCO}^{+}$ratios can become larger in XDRs than PDRs as well. Also, the HCN/CO 1-0 ratio is typically smaller in XDRs, and the HCN emission in XDRs is boosted with respect to CO only for high (column) density gas, with columns in excess of $10^{23} \mathrm{~cm}^{-2}$ and densities larger than $10^{4} \mathrm{~cm}^{-3}$. Furthermore, CO is typically warmer in XDRs than in PDRs, for the same total energy input. This leads to higher CO $J=N+1-N / C O 1-0, N \geq 1$, line ratios in XDRs. In particular, lines with $N \geq 10$, like $\mathrm{CO}(16-15)$ and $\mathrm{CO}(10-9)$ observable with HIFI/Herschel, discriminate very well between XDRs and PDRs. This is crucial since the XDR/AGN contribution will typically be of a much smaller (possibly beam diluted) angular scale and a 10-25\% PDR contribution can already suppress XDR distinguishing features involving HCN/HCO+ and $\mathrm{HNC} / \mathrm{HCN}$. For possible future observations, column density ratios indicate that $\mathrm{CH}, \mathrm{CH}^{+}, \mathrm{NO}, \mathrm{HOC}^{+}$and $\mathrm{HCO}$ are good $\mathrm{PDR} / \mathrm{XDR}$ discriminators.
\end{abstract}

Key words. galaxies: ISM - galaxies: active, X-rays: ISM - X-rays: galaxies

\section{Introduction}

The radiation that emanates from galaxy nuclei, such as those of NGC 253 and NGC 1068 or even more extreme (ultra-)luminous infrared galaxies, is believed to originate from regions with active star formation, an accretion disk around a central supermassive black hole or both (e.g. Silk 2005; Maloney 1999; Sanders \& Mirabel 1996). The unambiguous identification of the central energy source, or the relative contributions from stars and an active galaxy nucleus (AGN), remains a major challenge in the study of active galaxy centers. The general aim of this paper is to determine how the properties of the irradiated interstellar medium (ISM) may further our understanding.

Unlike emission at optical wavelengths, atomic, molecular and dust emission in the far-infrared and (sub-)mm range allow one to probe deeply into the large column densities of gas and

$\star$ Appendix A is only available in electronic form at http://www . aanda.org dust that occupy the centers of active galaxies. Observational studies of the ISM in galaxy centers have been presented by various authors (cf. Aalto 2005; Baan 2005; Ott et al. 2005; Israel 2005; Spoon et al. 2001; 2003; 2005; Klöckner et al. 2003; Israel \& Baas 2002; Garrett et al. 2001; Hüttemeister \& Aalto 2001; Curran et al. 2000). Theoretical models show that the spectral energy distribution of the radiation representing star formation (peaking in the ultraviolet) and AGN (peaking in the $\mathrm{X}$-ray regime) activity respectively influences the thermal and chemical balance of the ambient ISM in fundamentally different ways (Meijerink \& Spaans 2005; Maloney et al. 1996; Lepp \& Dalgarno 1996). The specific aim of this paper is thus to study the extent to which emission from commonly observed molecular and atomic line transitions may be used as a diagnostic tool in the study of external galaxy centers to determine the ambient conditions in general, and the type of irradiation in particular. 
Table 1. "Standard" clouds.

\begin{tabular}{llllll}
\hline \hline Type & $r(\mathrm{pc})$ & $n\left(\mathrm{~cm}^{-3}\right)$ & $N\left(\mathrm{~cm}^{-2}\right)$ & $G_{0}$ & $F_{\mathrm{X}}\left[\mathrm{erg} \mathrm{s}^{-1} \mathrm{~cm}^{-2}\right]$ \\
\hline A & 1 & $10^{4}-10^{6.5}$ & $3 \times 10^{22}-1 \times 10^{25}$ & $10^{2}-10^{5}$ & $1.6-160$ \\
B & 10 & $10^{3}-10^{4}$ & $3 \times 10^{22}-3 \times 10^{23}$ & $10^{1}-10^{4}$ & $1.6 \times 10^{-1}-16$ \\
C & 10 & $10^{2}-10^{3}$ & $3 \times 10^{21}-3 \times 10^{22}$ & $10^{0.5}-10^{3}$ & $1.6 \times 10^{-2}-1.6$ \\
\hline
\end{tabular}

To this effect, we have extended the chemical calculations described by Meijerink \& Spaans (2005; hereafter Paper I) for ultraviolet and X-ray irradiated gas to a much larger parameter space of ambient conditions and we have performed detailed radiative transfer calculations to compute the line intensities of many atomic and molecular transitions. We refer the interested reader to Paper I for a detailed description of the combined photon-dominated region (PDR)/X-ray dominated region (XDR) code that we have used to compute the impact of ultraviolet (PDR) and X-ray (XDR) photons on nearby ISM. The results described here will be applied to observations of external galaxy centers in a subsequent paper (Meijerink et al., in preparation). Stäuber et al. (2005) also developed PDR and XDR codes, for applications to Young Stellar Objects. The main difference is that this code has a time dependent chemistry and that it includes ice evaporation at $t=0$. This affects the chemistry compared with traditional steady-state models of pure gas-phase PDRs (e.g., CN/HCN). This is not relevant for our cloud models. However, freeze-out and evaporation start to become important for clouds with density $n_{\mathrm{H}} \geq 10^{5} \mathrm{~cm}^{-3}$.

\section{2. "Standard" clouds}

The current spatial resolution of sub-millimeter telescopes, such as the James Clerk Maxwell Telescope (JCMT), the Institute de Radio Astronomie Millimétrique (IRAM) telescope and even the Combined Array for Research in Submillimeter Astronomy (CARMA) or the Submillimeter Array (SMA), is insufficient to resolve individual clouds in extragalactic sources. By using these telescopes, each resolution element thus measures the combined emission from a large ensemble of molecular clouds. As a consequence, it is frequently impossible to use a single model cloud solution to describe the observed molecular lines. Instead, more complicated solutions involving two or more model clouds, with differing densities and incident radiation fields, are needed. This contrasts with the study of Galactic objects, where usually a single model cloud solution is sufficient to fit the measurements of single resolution elements.

In this paper, we calculate a grid of "standard" clouds sampling the different physical conditions believed to be relevant for the centers of active galaxy nuclei. In order to sample both the hierarchical size and (column) density properties of the ISM, we have chosen to construct models for a number of fixed sizes as well as densities. Note that the column densities are not the same for each model, since we use fixed cloud sizes. In these clouds, we investigate the detailed column density dependence for the line ratios $\mathrm{HCN} / \mathrm{CO}, \mathrm{HNC} / \mathrm{HCN}, \mathrm{HCO}^{+} / \mathrm{HCN}, \mathrm{SiO} / \mathrm{CO}$ and $\mathrm{CS} / \mathrm{HCN}$, which include line ratios observed in several galaxies. From our computational grid, one or more, properly weighted, models can be chosen to reproduce observed atomic and molecular lines.

We distinguish three different "standard" clouds, each with their own characteristic combinations of size and volume density range, hence also column density (cf. Table 1), for which we calculate a set of models for different incident radiation fields, and where a distinction between irradiation by far-ultraviolet (FUV) and $\mathrm{X}$-ray photons is made. The $\mathrm{X}$-ray radiation field is a powerlaw distribution $F(E)=F(0)(E / 1 \mathrm{keV})^{-\alpha}$ integrated between 1 and $100 \mathrm{keV}$, where $\alpha=-0.9$. A low energy cut-off of $1 \mathrm{keV}$ is chosen, since most of the emitted radiation below $1 \mathrm{keV}$ is absorbed in the hot, highly ionized medium close to the black hole. This power law spectrum is generally believed to be representative for accreting black holes. Please note that this differs from Paper I, where we used a thermal distribution at $10^{6} \mathrm{~K}$ instead. The ultraviolet radiation field $(6-13.6 \mathrm{eV})$ is expressed in multiples of the Habing flux, where $G_{0}=1$ corresponds to $1.6 \times 10^{-3} \mathrm{erg} \mathrm{cm}^{-2} \mathrm{~s}^{-1}$, which is the local Galactic interstellar radiation field. We express the X-ray flux in $\mathrm{erg} \mathrm{s}^{-1} \mathrm{~cm}^{-2}$. An accreting black hole with an X-ray luminosity of $10^{44} \mathrm{erg} \mathrm{s}^{-1}$, produces an X-ray flux of $100 \mathrm{erg}^{-1} \mathrm{~cm}^{-2}$ at a distance of $100 \mathrm{pc}$, when there is not extinction. We use a line width $\delta_{v}=2.7 \mathrm{~km} \mathrm{~s}^{-1}$. Cloud type A represents compact, high-density environments such as molecular cloud cores, and clouds very close to active nuclei; cloud type $\mathrm{B}$ corresponds to more traditional molecular cloud environments, and cloud type $\mathrm{C}$ is representative of the more diffuse extended (molecular) medium in which clouds of type B are usually embedded.

Late-type galaxies frequently have radial metallicity gradients, with the highest metallicity in the center (Vila-Costas \& Edmunds 1992; Zaritsky et al. 1994). For this reason, we have adjusted the metallicity used in Table 2 of Paper I. The published metallicity gradients and suggestions of a gradient flattening in the very center have led us to adopt a twice Solar metallicity as a reasonable value. Since $[\mathrm{C}] /[\mathrm{O}]$ abundance ratios decrease at higher metallicities, we have taken the carbon abundance equal to the oxygen abundance (see, for instance, Garnett et al. 1999; Kobulnicky \& Skillman 1998). Note that the $[\mathrm{C}] /[\mathrm{O}]$ ratio affects the abundances of $\mathrm{O}_{2}$ and $\mathrm{H}_{2} \mathrm{O}$. See for example Spaans $\&$ van Dishoeck (2001) and especially Fig. 2 in Bergin et al. (2000).

From the models, we have calculated the intensities of the molecular rotational lines of $\mathrm{HCN}, \mathrm{HNC} \mathrm{HCO}^{+}, \mathrm{CS}$ and $\mathrm{SiO}$ (upto $J=4$ ), [CI], [CII], [OI], [SiII] and other fine-structure lines. For $\mathrm{CO}$ and ${ }^{13} \mathrm{CO}$ we calculated the intensities of the rotational lines up to $J=16$, in order to make predictions for future observing facilities such as the ESO Herschel/HIFI space mission. We use the Leiden Atomic and Molecular Database (LAMDA) as described in Schöier et al. (2005) to retrieve the collisional data needed for the calculations. Where no collisional data are available for commonly observed molecules such as $\mathrm{CN}$, $\mathrm{CH}, \mathrm{CH}^{+}, \mathrm{HCO}, \mathrm{HOC}^{+}$, $\mathrm{NO}$ and $\mathrm{N}_{2} \mathrm{H}^{+}$, we only give column densities.

\section{Surface temperatures}

To illustrate the coupling differences of FUV and X-ray photons to the gas, we first discuss the surface temperatures of the low, mid, and high density models respectively over the parameter space given for the PDR and XDR models in Table 1. We calculated a larger range of radiation fields for the PDR models than for the XDR models, for reasons related to the heating efficiency 


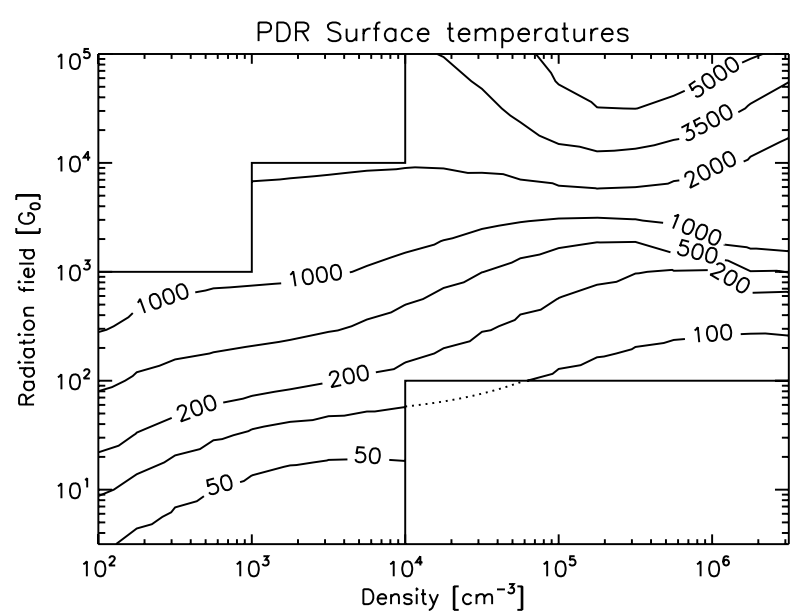

XDR Surface temperatures

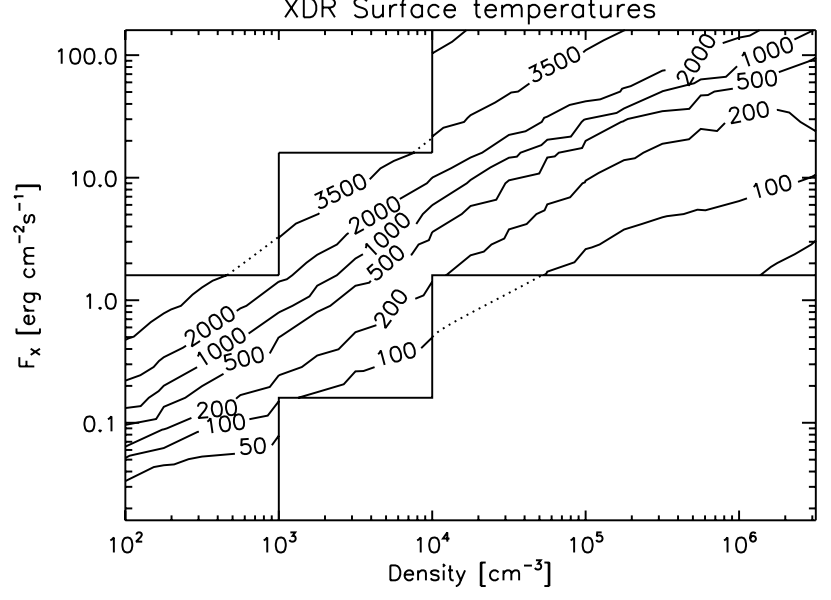

Fig. 1. Surface temperatures for PDR (top) and XDR (bottom) models.

which are discussed below. The resulting surface temperatures as a function of gas density and incident radiation intensity for both PDR and XDR models are shown in Fig. 1.

The most important heating mechanism at the edge of a PDR is photo-electric heating. FUV photons are absorbed by dust grains, which release electrons that lose their surplus kinetic energy to the gas by Coulomb interactions. The efficiency of this process increases, when the grains are more negatively charged, which is determined by a complex interplay of the impinging radiation field $G_{0}$, electron density $n_{\mathrm{e}}$ and gas temperature $T$. The absolute efficiency is very low, since about $0.5-3$ percent of the photon energy goes into gas heating. This sharply contrasts with direct X-ray heating, important at the edge of XDRs. Direct ionization of an atom yields a kinetic electron with an energy higher than $1 \mathrm{keV}$. This electron heats, ionizes and excites the gas. Depending on the $\mathrm{H}, \mathrm{H}_{2}, \mathrm{He}$ and electron abundances, the heating efficiency can be up to 70 percent, much higher than for photo-electric heating. However, there is an opposing effect, namely the much lower absorption cross section for X-rays. Since the cross sections scale roughly as $E^{-3}$, there are many fewer X-ray photons absorbed than FUV photons.

We find that at high densities $\left(n>10^{4} \mathrm{~cm}^{-3}\right.$ ) PDR models produce higher surface temperatures than the XDR models. At low densities, however, we find the opposite, especially in the case of high radiation fields. This is explained by the drop in the efficiency of photo-electric heating due to grain charging at densities $n<10^{5} \mathrm{~cm}^{-3}$ when the same impinging radiation field is considered. This is nicely illustrated in Fig. 6 of
Kaufman et al. (1999), where the ratios of the intensity of the [CII] $158 \mu \mathrm{m}$ and [OI] $63 \mu \mathrm{m}$ lines to the total far-infrared intensity emitted from the surface of the clouds are plotted as a function of density and radiation field. This ratio is a measure of the heating efficiency, since [CII] $158 \mu \mathrm{m}$ and [OI] $63 \mu \mathrm{m}$ are the most important coolants in PDRs.

In the regime discussed here, the surface temperatures of the XDR models are quite well correlated with $H_{\mathrm{X}} / n$, where $H_{\mathrm{X}}$ is the energy deposition per particle and $n$ the total hydrogen density. This means that the highest surface temperatures are found at the lowest densities and highest impinging radiation fields. Consequently, the contours of equal surface temperature are almost straight lines in the XDR plot of Fig. 1. In the PDR models their behavior is more complicated, as already discussed by Kaufman et al. (1999), since grains are involved in heating the gas. At the edge of the cloud, the cooling is dominated by [CII] $158 \mu \mathrm{m}$ and [OI] $63 \mu \mathrm{m}$, which have critical densities of $n_{\mathrm{cr}}(\mathrm{CII}) \approx 3 \times 10^{3} \mathrm{~cm}^{-3}$ and $n_{\mathrm{cr}}(\mathrm{OI}) \approx 5 \times 10^{5} \mathrm{~cm}^{-3}$ for collisions with atomic and molecular hydrogen. The cooling rate in this regime is more or less proportional to $n^{2}$. The heating rate is at least proportional to $n$, because the grain density is proportional to $n$. It can be larger, because grains become less positively charged at increasing electron densities making it easier for electrons to escape the grains, so that the heating efficiency increases. Nevertheless, the density dependence of heating remains less steep than $n^{2}$, i.e., less than that of cooling, which causes a drop in the temperature at $n<10^{4} \mathrm{~cm}^{-3}$ and for fixed $G_{0}$ with increasing density. Between $n \approx 1-3 \times 10^{3} \mathrm{~cm}^{-3}$ where the [CII] $158 \mu \mathrm{m}$ line thermalizes, the drop in temperature stagnates. For densities between $n=10^{4}-10^{5} \mathrm{~cm}^{-3}$ and $G_{0}<10^{4}$, we also find that the surface temperature drops with increasing $n$. The [OI] $63 \mu \mathrm{m}$ line thermalizes at higher densities $n=10^{5}-10^{6} \mathrm{~cm}^{-3}$ and, therefore, cooling will be proportional to $n$. Heating increases faster with density, which results in a higher surface temperature. For $G_{0}>10^{4}$, we find that the surface temperature rises up to $n \approx 5 \times 10^{5} \mathrm{~cm}^{-3}$ due to the increase in the heating efficiency with density at fixed $G_{0}$. Above this density and at these high temperatures, coolants with high critical densities and excitation energies such as the [OI] $6300 \AA$ line become important, causing the surface temperature to drop again.

When we compare the surface temperatures with those derived by Kaufman et al. (1999), we find that our model surface temperatures are higher. A possible explanation for this has been discussed recently by Röllig et al. (2006), who present scaling relations for heating and cooling as a function of metallicity $Z$. They state that the photo-electric heating rate is $\propto Z$ for $n<10^{3} \mathrm{~cm}^{-3}$ increasing to $Z^{2}$ when $n>10^{6} \mathrm{~cm}^{-3}$. The cooling rate is always proportional to $Z$, and, therefore, higher metallicities result in higher surface temperatures. Note however that the temperature differences found are very likely not only because of a change in metallicity. In the PDR comparison test (Röllig et al. 2006), we found significant scatter between different PDR codes in the thermal balance. Therefore, one should not take the absolute values of line intensities too literally in the interpretation of data.

\section{Fine-structure lines}

In principle, we can use combinations of fine-structure line intensities to constrain densities and incident radiation fields. Here we discuss how such line ratios depend on gas density, ambient radiation field strength, and cloud column density, and compare the results for the PDR and XDR models. In previous studies only results for PDRs were shown. Wolfire et al. (1990) were 
the first to show [CII]/[SiII] contour plots for PDRs. Recently, Kaufman et al. (2006) showed updated plots for [SiII] and [FeII] for PDR gas. The fine-structure lines are selfconsistently calculated in the PDR and XDR codes, and we take line-trapping into account for ALL lines.

\section{1. [Sill] $35 \mu \mathrm{m} /[\mathrm{CII}] 158 \mu \mathrm{m}$ intensity ratio}

In Fig. A.1, we show the [SiII] $34.8 \mu \mathrm{m} /[\mathrm{CII}] 158 \mu \mathrm{m}$ finestructure line ratio for both the PDR and the XDR models. The [SiII] $34.8 \mu \mathrm{m}$ line has an energy of $E / k=414 \mathrm{~K}$ and a critical density of $n_{\mathrm{cr}}=3.4 \times 10^{5} \mathrm{~cm}^{-3}$, while this is $E / k=92 \mathrm{~K}$ and $n_{\mathrm{cr}}=2.8 \times 10^{3} \mathrm{~cm}^{-3}$ for the [CII] $158 \mu \mathrm{m}$. Very high radiation fields produce gas temperatures in the PDR models that are sufficiently high to excite the [SiII] and [CII] upper states and the ratio depends mostly on density. With lower radiation fields, the surface temperature drops. The upper level energy of [SiII] $34.8 \mu \mathrm{m}$ is reached first and the [SiII]/[CII] line ratio drops. As the density increases at a given FUV radiation field, the ratios limit to roughly constant values that are set by the corresponding surface temperatures.

In the XDR models, the ratio is not only determined by the temperature and density, but also by the fractional abundances and column densities. In PDRs clearly defined layers occur, in which carbon and silicon are both almost fully ionized, but throughout XDRs neutral and ionized species co-exist. Despite the fact that surface temperatures in the XDR models are lower, we find much higher [SiII]/[CII] ratios, since silicon is much easier to ionize than carbon. The dominant source for ionization is not the direct absorption of an X-ray photon (primary ionization), but the produced kinetic electron. This electron can ionize a species either directly by collisions (secondary ionizations) or indirectly by first exciting $\mathrm{H}$ and $\mathrm{H}_{2}$ and producing Lyman $\alpha$ and Lyman-Werner photons, which then may ionize species in turn. The cross section of Si for secondary ionization is about twice that of $\mathrm{C}$. This does not, however, fully explain the order of magnitude difference between the calculated PDR and XDR ratios. This difference also reflects the fact that ionization of $\mathrm{C}$ can only be done by Lyman-Werner photons, whereas both Lyman $\alpha$ and Lyman-Werner photons are capable of ionizing Si. It is thus harder to ionize $\mathrm{C}$ than $\mathrm{Si}$ in regions where the gas is mostly atomic, which results in a large increase of the ratio for all densities at a given irradiation strength.

This also explains the fairly constant ratio below $F_{\mathrm{X}} \approx$ $10 \mathrm{erg} \mathrm{s}^{-1} \mathrm{~cm}^{-2}$ for $n \sim 2 \times 10^{3}-3 \times 10^{6} \mathrm{~cm}^{-3}$, which results from an interplay between various effects. With ambient radiation fields constant, we find that the surface temperatures drop with increasing density in the XDR. At lower temperatures and higher densities, $\mathrm{H}_{2}$ is more easily formed. Both the temperature drop itself and the enhanced $\mathrm{H}_{2}$ (leading to more carbon ionizations) thus suppresses the $[\mathrm{SiII}] /[\mathrm{CII}]$ ratio, but this is compensated by the relatively high critical density of the [SiII] $34.8 \mu \mathrm{m}$ line.

For the highest, $>16 \mathrm{erg} \mathrm{s}^{-1} \mathrm{~cm}^{-2}$, radiation fields, where carbon is highly ionized, we find the same trend as seen for the PDR, i.e., the ratio is mostly dependent on density for the highest radiation fields, and then there is a decrease in the ratio when the temperature drops below the upper-state energy of [SiII].

At the lowest densities and highest X-ray radiation fields, we find that the effect of column density become important as well. Since we fixed cloud sizes, in each cloud type the lowest density models imply also the lowest column densities. In the high irradiation models, carbon is almost fully ionized at the XDR edge. The fractional abundance of ionized carbon drops toward the $\mathrm{H} / \mathrm{H}_{2}$ transition and then increases again for the reasons discussed earlier. In the lowest (column) density models, we only produce the highly ionized part, suppressing the ratio even more. This is best seen in the diagram for the high density XDR models, at densities $n=10^{4} \mathrm{~cm}^{-3}$. The ratio increases from $F_{\mathrm{X}}=1.6$ to $30 \mathrm{erg} \mathrm{s}^{-1} \mathrm{~cm}^{-2}$ and then drops again. Hence, if we increase the column densities of these models by a factor of $\sim 30$, the ratios only depend on radiation field strength and not on density.

\section{2. $[\mathrm{OI}] 63 \mu \mathrm{m} /[\mathrm{CII}] 158 \mu \mathrm{m}$ intensity ratio}

In Fig. 2, we show the [OI] $63 \mu \mathrm{m} /[\mathrm{CII}] 158 \mu \mathrm{m}$ ratio. The critical density is $n_{\mathrm{cr}}=5 \times 10^{5} \mathrm{~cm}^{-3}$ and the upper-state energy is $E / k=228 \mathrm{~K}$ for [OI] $63 \mu \mathrm{m}$. In the PDR models and at very high incident radiation fields, this ratio depends mostly on density as in the case for the $[\mathrm{SiII}] /[\mathrm{CII}]$ ratio, since once again temperatures are sufficiently high to excite both upper-state levels. When the surface temperature drops for lower radiation fields, the upper-state energy of $[\mathrm{OI}]$ is reached first. The $[\mathrm{OI}] /[\mathrm{CII}]$ ratio then drops, with a flat density dependence for $n<10^{4} \mathrm{~cm}^{-3}$ and $G_{0}<10^{3}$. The decrease in this line ratio is, however, less pronounced than that in the $[\mathrm{SiII}] /[\mathrm{CII}]$ ratio, because the upperstate energy of [OI] is lower than that of [SiII]. When $G_{0}<10^{3}$ and $n>10^{5.5} \mathrm{~cm}^{-3}$, the ratio is roughly constant at the same density for all values of $G_{0}$. Here, the more difficult excitation of $[\mathrm{OI}]$ at lower temperatures is counteracted by the $\mathrm{C}^{+}$layer becoming thinner at lower radiation fields.

In the $\mathrm{XDR}$, the $[\mathrm{OI}] /[\mathrm{CII}]$ ratio is again more complex due to its dependence on density, radiation field, ionized carbon fraction and total column density. The ratios are overall much higher than in the PDR models, since carbon does not become fully ionized. At the largest $H_{\mathrm{X}} / n$, temperatures are high enough to create a dependence on density only. At lower radiation fields, the $[\mathrm{OI}] /[\mathrm{CII}]$ ratio drops as temperatures approach the upper-state energy of the [OI] $63 \mu \mathrm{m}$ line. When we decrease the radiation field even more, we find that the $\mathrm{C}^{+}$fraction is rapidly reduced at high $\left(>10^{5} \mathrm{~cm}^{-3}\right)$ densities and the ratio increases again. At high $\left(>16 \mathrm{erg} \mathrm{s}^{-1} \mathrm{~cm}^{-2}\right)$ radiation field strength and low densities, a large $H_{\mathrm{X}} / n$ is maintained throughout the whole cloud, since the relevant type A cloud size is only one parsec. For that reason, we find here the same effect as already seen in the [SiII]/[CII] ratio, since column densities increase toward higher densities. At the highest radiation fields and lowest densities, the ratio is suppressed since carbon remains partially ionized over the full extent of the clouds considered here. We do not find the highest ratio at the highest radiation field in the lower density models.

\section{3. [Fell] $26 \mu \mathrm{m} /[\mathrm{CII}] 158 \mu \mathrm{m}$ intensity ratio}

In Fig. A.2, we show the [FeII] $26 \mu \mathrm{m} /[\mathrm{CII}] 158 \mu \mathrm{m}$ intensity ratio. [FeII] $26 \mu \mathrm{m}$ is very difficult to excite due to its high critical density $n_{\mathrm{cr}}=2.2 \times 10^{6} \mathrm{~cm}^{-3}$ and upper-state energy $E / k=554 \mathrm{~K}$. Thus, the change in ratio with increasing density at high incident radiation fields is much larger than that seen for the [SiII] $34.8 \mu \mathrm{m} /[\mathrm{CII}] 158 \mu \mathrm{m}$ ratio. In the PDR models and at high incident radiation fields, the ratio mostly depends on the density. At lower radiation field strengths, approaching the upper-state energy of [FeII], the ratio drops.

The same trends are seen at high radiation fields in the XDR models, but again, we find much higher ratios than in the PDR models. It is possible to ionize iron with Lyman $\alpha$ photons, but not carbon. At moderate radiation fields, we again find ratios to be more or less independent of density, for the reason that 

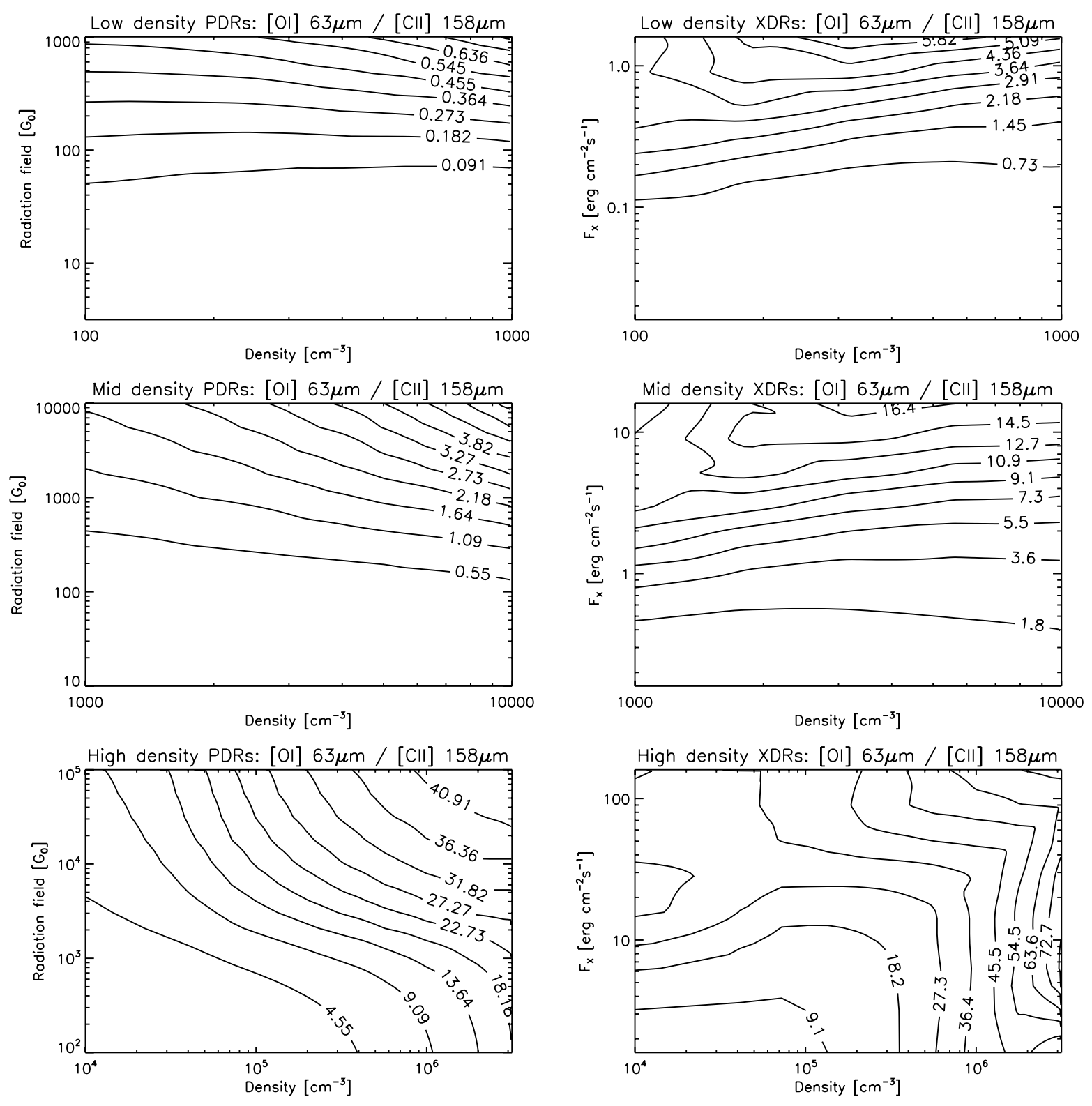

Fig. 2. [OI] $63 \mu \mathrm{m} /[\mathrm{CII}] 158 \mu \mathrm{m}$ ratio for PDR (left) and XDR (right) models.

we have already discussed in the $[\mathrm{SiII}] /[\mathrm{CII}]$ case. At the lowest densities and highest radiation fields in each cloud type, we find that the ratios are similarly suppressed as was the case for [SiII] $35 \mu \mathrm{m} /[\mathrm{CII}] 158 \mu \mathrm{m}$ and [OI] $63 \mu \mathrm{m} /[\mathrm{CII}] 158 \mu \mathrm{m}$.

\section{4. $[\mathrm{Cl}] 369 \mu \mathrm{m} /[\mathrm{Cl}] 609 \mu \mathrm{m}$ intensity ratio}

In Fig. 3, we show the [CI] $369 \mu \mathrm{m} /[\mathrm{CI}] 609 \mu \mathrm{m}$ intensity ratio. The critical densities of these lines are $n_{\mathrm{cr}}=3 \times 10^{2} \mathrm{~cm}^{-2}$ for $[\mathrm{CI}]$ $609 \mu \mathrm{m}$ and $n_{\mathrm{cr}}=2 \times 10^{3} \mathrm{~cm}^{-2}$ for [CI] $369 \mu \mathrm{m}$, typically lower than the densities we are considering here. The upper-state energies are $E / k=24$ and $63 \mathrm{~K}$ for [CI] $609 \mu \mathrm{m}$ and [CI] $369 \mu \mathrm{m}$, respectively. In the PDR models, the $[\mathrm{CI}]$ lines originate from the $\mathrm{C}^{+} / \mathrm{C} / \mathrm{CO}$ transition layer. The temperatures in this layer slightly rise with increasing incident radiation field strengths and range between $T=20-100 \mathrm{~K}$ (comparable to the upper-state energies). This explains the small increase in the ratio for larger $G_{0}$ at the same density. The ratio does not change much as a function of density, since we are above the critical density. The change we do see, however, has a temperature dependence. When densities are lowered, recombination rates are lower as well. By consequence, at higher densities, the transition layer is closer to the edge of the cloud and at higher temperatures, which raises the ratio.

In the XDR, neutral carbon occurs throughout the cloud, and is also abundant at relatively high temperatures. The spread in temperatures is large, which was already seen in Sect. 3, and this determines for a large part differences in the ratios. The temperature of a cloud is determined by $H_{\mathrm{X}} / n$, resulting in the highest ratios for low $n$ and high $F_{\mathrm{X}}$, opposite to the situation in the PDR models. The three cloud types, with their different low, mid and high density ranges nevertheless show very similar spreads in ratios. This is caused by the difference in column densities, which also has a very important effect. The low, mid, and high density models have their own fixed cloud size, and in each standard cloud type, column densities increase toward higher densities in the same density range. The higher density models contain larger regions of low temperature, which suppresses the ratio at these 

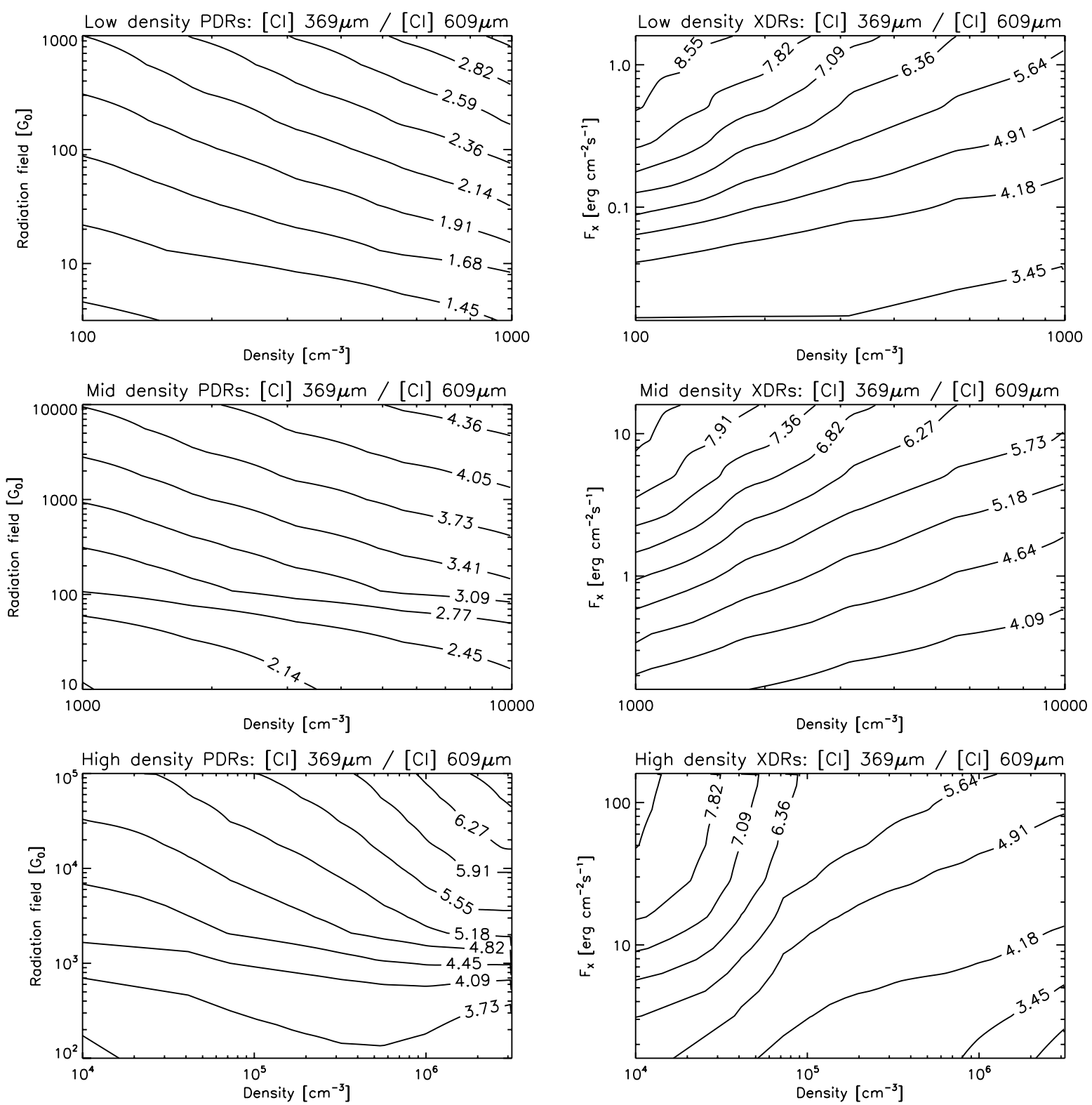

Fig. 3. $[\mathrm{CI}] 369 \mu \mathrm{m} /[\mathrm{CI}] 609 \mu \mathrm{m}$ ratio for PDR (left) and XDR (right) models.

densities even more. This can also be understood by considering the ratio at $n=10^{4} \mathrm{~cm}^{-3}$ in the mid (type B) and high (type C) density range. The high density model at $n=10^{4} \mathrm{~cm}^{-3}$ has a smaller cloud size and therefore a higher line ratio.

\section{Rotational lines}

Molecular rotational lines are also characteristic for the physical condition of ISM gas and may also be used to constrain gas densities and incident radiation fields. In the following, we discuss a number of ratios, involving the molecular species ${ }^{12} \mathrm{CO},{ }^{13} \mathrm{CO}$, $\mathrm{HCN}, \mathrm{HNC}, \mathrm{HCO}^{+}, \mathrm{SiO}$ and $\mathrm{CS}$. Although we reproduce in this Paper only a limited number of the diagrams showing the calculated line intensity ratios, all model data are available on-line ${ }^{1}$. Hence, the reader can determine all line ratios and integrate over all possible lines of sight as interest dictates. Here we concentrate on molecular lines that we consider particularly useful to

${ }^{1}$ http://www.strw.leidenuniv.nl/ meijerin/grid/ distinguish between PDRs and XDRs. We have calculated the line intensities by using a one-dimensional version of the radiation transfer code described in Poelman \& Spaans $(2005,2006)$.

\subsection{CO rotational lines}

In Fig. A.3, we show the $\mathrm{CO}(1-0)$ line intensity for cloud types A (high-density), B (mid-density) and C (low-density) for both the PDR and XDR model cases (cf. Table 1). All three cloud types are relevant for $\mathrm{CO}$ (and ${ }^{13} \mathrm{CO}$ ) since these molecules are present ubiquitously on all galactic scales. The $\mathrm{CO}(1-0)$ line has an upper state energy $E / k=5.53 \mathrm{~K}$ and a critical density $n_{\mathrm{cr}} \sim 3 \times 10^{3} \mathrm{~cm}^{-3}$. In the low density PDR models $\left(n=10^{2}-10^{3} \mathrm{~cm}^{-3}\right)$, we find that the intensity increases with density only. In the mid $\left(n=10^{3}-10^{4} \mathrm{~cm}^{-3}\right)$ and high ( $n=10^{4}-10^{6.5} \mathrm{~cm}^{-3}$ ) density range, we also find a small line intensity increase at higher incident radiation fields. The emission of the $\mathrm{CO}(1-0)$ line is optically thick and the emitted line intensity mostly depends on the gas temperature near the $\tau=1$ 

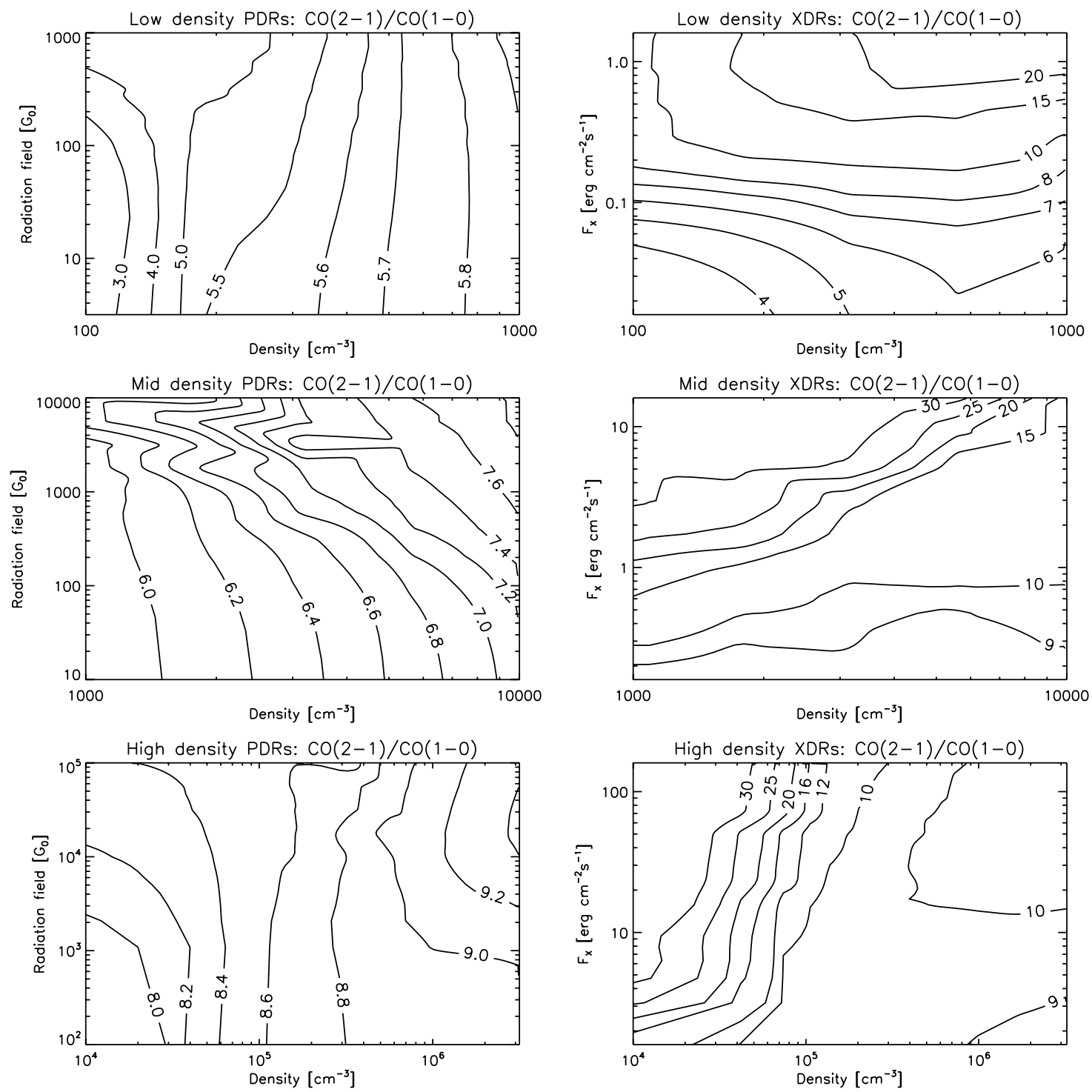

Fig. 4. $\mathrm{CO}(2-1) / \mathrm{CO}(1-0)$ ratio for PDR (left) and XDR (right) models.

surface. Although the upper-state energy is below the gas temperature, we find a significant increase in the line intensity. At higher densities, gas-grain interactions may significantly increase gas temperatures in the highly attenuated part of the cloud. Since the escape probability $\beta(\tau) \propto 1 / \tau$ when $\tau>1$, these parts also add a small contribution to the line intensity, which is proportional to $\sim \ln (\tau)$. For an elaborate discussion of the $\mathrm{CO}(1-0)$ line intensity dependence on $G_{0}$ and $n$ see Wolfire et al. (1989). In general, the line intensities do not vary much in the PDR models, as opposed to what is seen in the XDR models. In all PDR models, the $\mathrm{CO}(1-0)$ lines are optically thick. In the XDR models for type $\mathrm{C}$ clouds $\left(n=10^{2}-10^{3} \mathrm{~cm}^{-3}\right)$, the line intensities vary over two orders of magnitude. Because of the fixed cloud size, models at higher densities have larger column densities. Even at the point farthest from the cloud edge, the low density gas in type $\mathrm{C}$ clouds causes relatively little radiation attenuation, and with high incident radiation it is at very high temperature and in a highly ionized state throughout. Under these conditions warm $\mathrm{CO}$ gas present, but only in very small amounts. Therefore, at such points in parameter only very weak line emission is produced and the lines are optically thin. In the high density range $\left(n=10^{4}-10^{6.5} \mathrm{~cm}^{-3}\right)$ of type A clouds, on the other hand, the spread in intensity is much reduced. Although at densities of $n=10^{4} \mathrm{~cm}^{-3}$ most of the cloud is still at a high temperature and in a highly ionized state, there is sufficient column density to have $\mathrm{CO}$ abundances large enough to produce significant line emission. At even higher densities $\left(n=10^{6} \mathrm{~cm}^{-3}\right)$, the column densities are high enough to attenuate the radiation field in such a way that a large CO fraction is produced $\left(\sim 10^{-4}\right)$, but still at a temperature of $T \sim 100 \mathrm{~K}$. Here, the $\mathrm{CO}(1-0)$ line emission produced in XDRs can be two to four times stronger than that in PDRs.

For the $\mathrm{CO}(2-1)$ line, the upper state energy is $E / k=$ $16.60 \mathrm{~K}$ and the critical density is $n_{\mathrm{cr}} \sim 1 \times 10^{4} \mathrm{~cm}^{-3}$. Although not shown, the line intensities exhibit a behavior as a function of density and radiation field very similar that that of the $\mathrm{CO}(1-0)$ line. Because of the higher upper state energy, we find a somewhat stronger dependence on radiation field in the PDR models. The effect of the larger critical density is hard to see, due to the large optical depths, but do show up when we consider the $\mathrm{CO}(2-1) / \mathrm{CO}(1-0)$ ratio. This ratio is shown in Fig. 4. In the PDR models, the ratio does not differ more than a factor of two over the full density range considered here. In the XDR models, very large line intensity ratios of 30 or more are found, especially at 

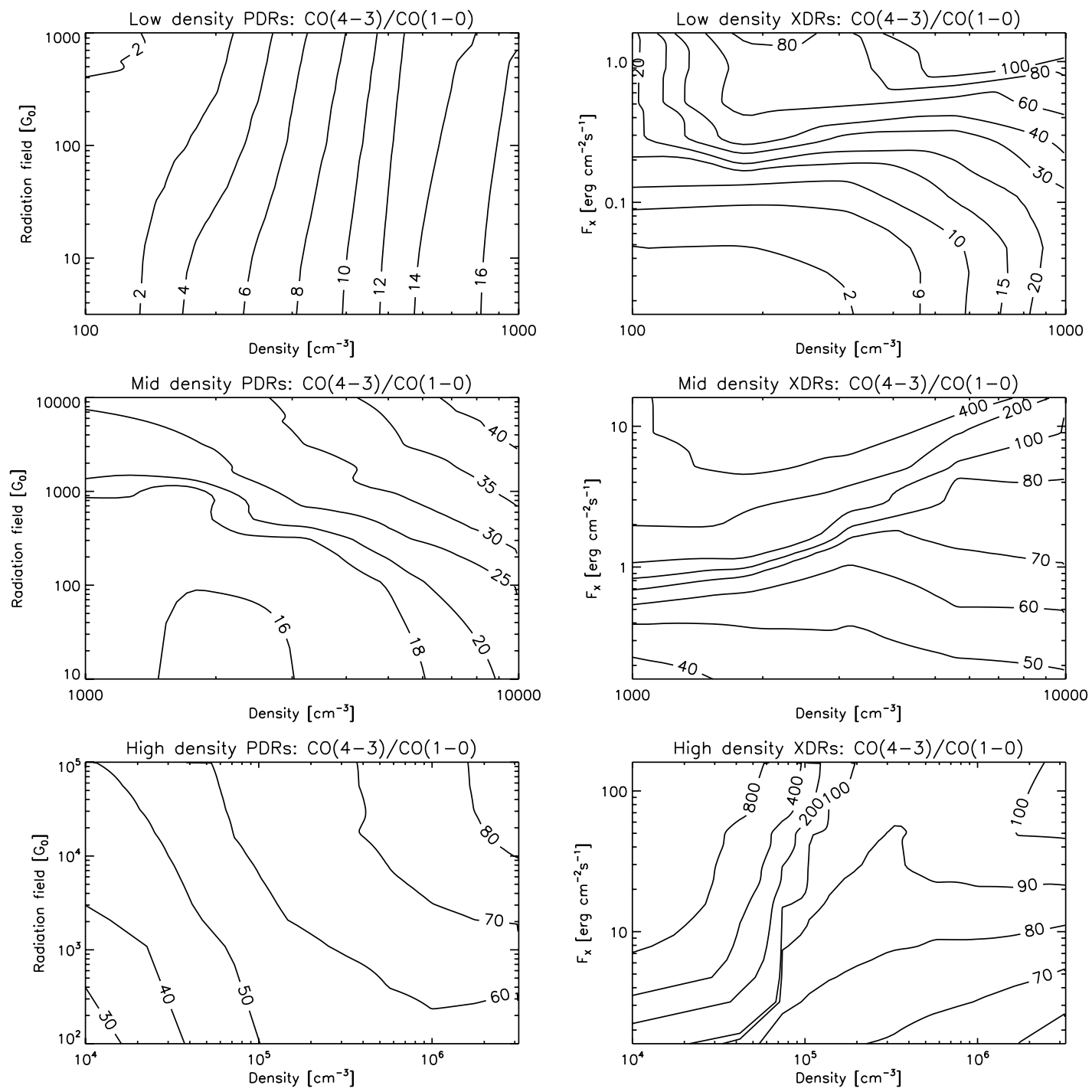

Fig. 5. $\mathrm{CO}(4-3) / \mathrm{CO}(1-0)$ ratio for PDR (left) and XDR (right) models.

high incident radiation fields. It is, however, very questionable whether we will actually observe these high ratios, since the intensity of the emitted emission is low. The $\mathrm{CO}(2-1) / \mathrm{CO}(1-0)$ ratio dependence on density and radiation field is in general weak, especially in PDRs, since the upper state energies are not very high and the difference in critical density is small.

The CO(4-3) line (see Fig. A.4) has an upper state energy $E / k=55.32 \mathrm{~K}$ and critical density $n_{\mathrm{cr}} \sim 4 \times 10^{4} \mathrm{~cm}^{-3}$. As expected, the emitted intensity shows more variation with density and radiation field. In Fig. 5, we show the $\mathrm{CO}(4-3) / \mathrm{CO}(1-0)$ line intensity ratio. In the low density range (cloud type $\mathrm{C}$ ), PDR models still produce a line ratio increase only as function of density, but the variation in the ratio has grown to more than a factor of two, as opposed to only 20 percent in the corresponding $\mathrm{CO}(2-1) / \mathrm{CO}(1-0)$ ratio. The XDRs for this cloud type show a complex behavior with density and radiation field and the ratios cover a much larger range from about 2 to 40 . At low radiation fields, only a density dependence is seen. At high radiation field strengths, the effect of the column density comes into play. In the mid density (type B) PDRs, the highest line ratios are seen for the highest densities and radiation fields. The XDRs in this range show only a dependence on radiation field. The effect of the higher density is compensated by the fact that at lower densities relatively more gas is at high temperatures. The gas temperature plays a large role in the density range applicable to this cloud type. $\mathrm{CO}$ is present at much higher temperatures in the XDRs. Therefore, the XDR line ratios for the same density and incident radiation field can be more than ten times larger than in the PDR. This difference slowly disappears when the critical density of the $\mathrm{CO}(4-3)$ line is reached and the $\mathrm{CO}(4-3)$ line also thermalizes, which is seen in the high density range (cloud type A) at densities $n>10^{5} \mathrm{~cm}^{-3}$.

\subsection{High $\mathrm{J}-\mathrm{CO}$ rotational transitions}

In cloud type A XDRs $\left(n=10^{4}-10^{6.5} \mathrm{~cm}^{-3}\right), \mathrm{CO}$ is present throughout the cloud, even when energy deposition rates $H_{\mathrm{X}} / n$ are large and temperatures are high $(T \sim 200 \mathrm{~K})$. This warm $\mathrm{CO}$ gas produces emission originating from high rotational transitions even when densities are not high (e.g., $n=10^{4} \mathrm{~cm}^{-3}$ ). Contrary to the situation in XDRs, most CO in PDRs is produced beyond the $\mathrm{H} / \mathrm{H}_{2}$ transition and it has on average much lower temperatures $(T \sim 20-50 \mathrm{~K})$, causing lower intensities and line ratios. Therefore, it is very likely, that future missions such as Herschel/HIFI will be able to distinguish between 

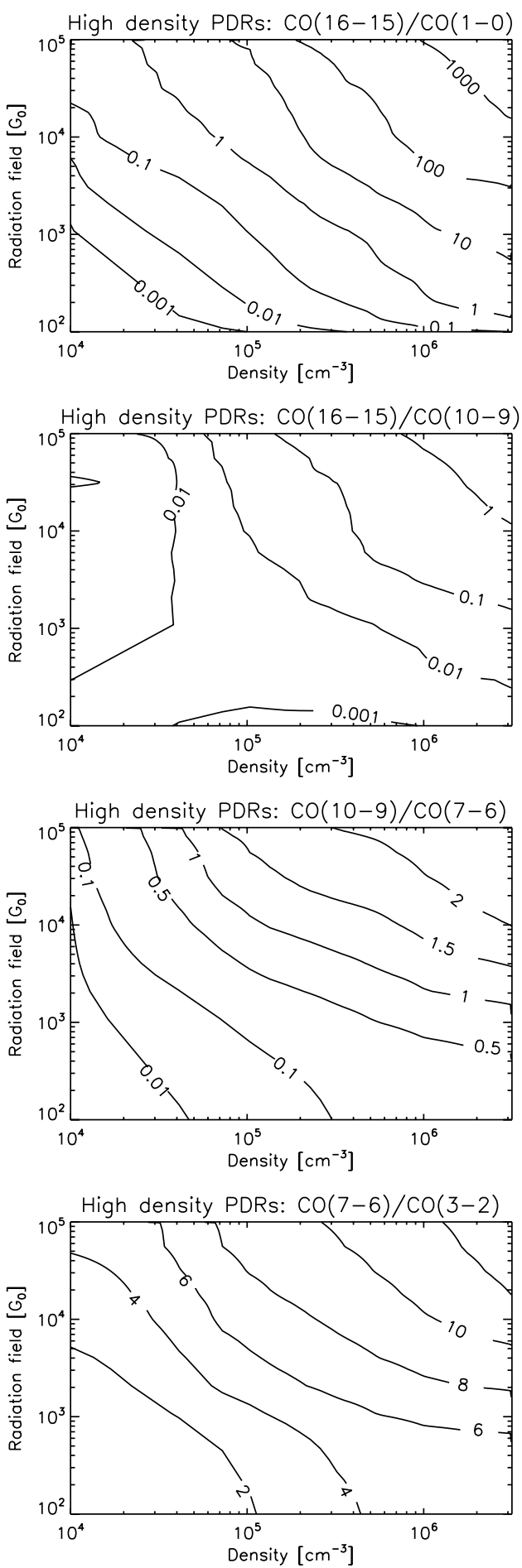
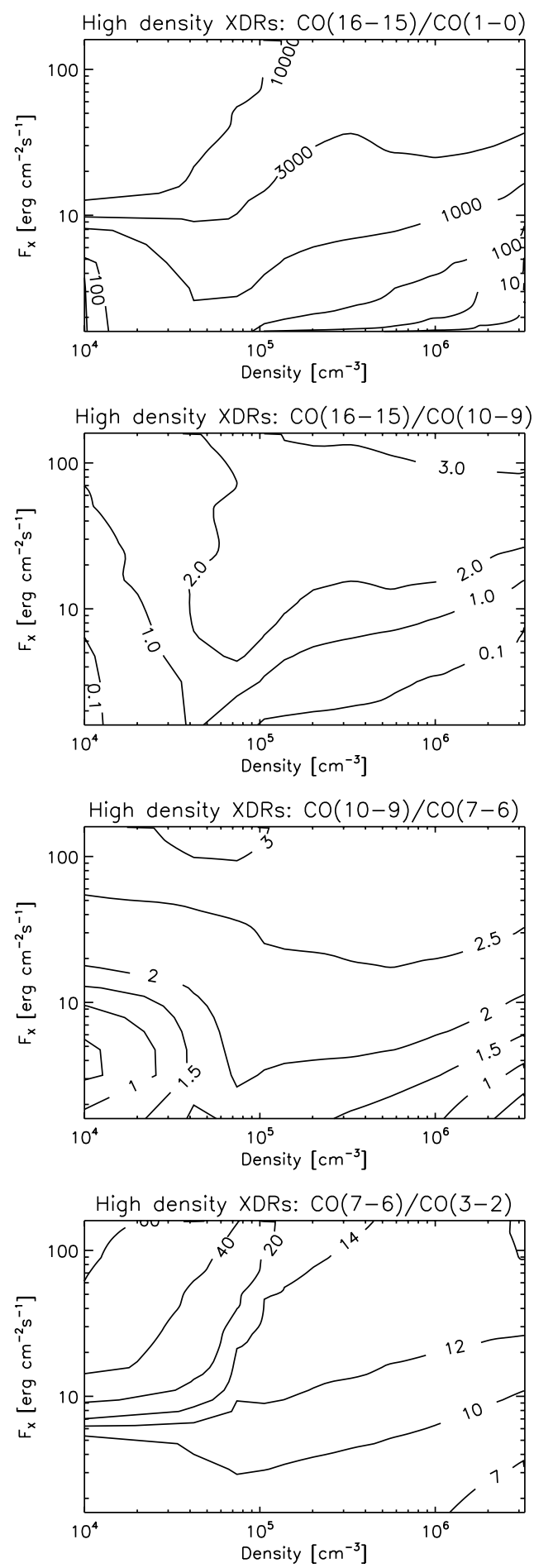

Fig. 6. $\mathrm{CO}(16-15) / \mathrm{CO}(1-0), \mathrm{CO}(16-15) / \mathrm{CO}(10-9), \mathrm{CO}(10-9) / \mathrm{CO}(7-6)$ and $\mathrm{CO}(7-6) / \mathrm{CO}(3-2)$ ratio for $\mathrm{PDR}($ left $)$ and XDR (right) models.

PDRs and XDRs, by observing high rotational transitions such as $\mathrm{CO}(16-15), \mathrm{CO}(10-9)$, and $\mathrm{CO}(7-6)$.

In Fig. A.5, we show the PDR and XDR intensities of the $\mathrm{CO}(7-6), \mathrm{CO}(10-9)$ and $\mathrm{CO}(16-15)$ lines for the high density range (cloud type A). We find that for both PDRs and XDRs, the spread in intensities increases for higher rotational lines, since the critical densities of these transitions are higher. However, this spread is much larger for PDRs than for XDRs. The CO(16-15) line intensity ranges from $\sim 10^{-10}\left(n=10^{4} \mathrm{~cm}^{-3}\right.$ and $\left.G_{0}=10^{2}\right)$ to $\sim 10^{-3} \mathrm{erg} \mathrm{s}^{-1} \mathrm{~cm}^{-2} \mathrm{sr}^{-1}\left(n=10^{6.5} \mathrm{~cm}^{-3}\right.$ and $\left.G_{0}=10^{5}\right)$ for the PDR models, while this is $\sim 10^{-4}\left(n=10^{4} \mathrm{~cm}^{-3}\right)$ to $\sim 10^{-2} \mathrm{erg} \mathrm{s}^{-1} \mathrm{~cm}^{-2} \mathrm{sr}^{-1}\left(n=10^{6.5} \mathrm{~cm}^{-3}\right)$ for the XDR models. PDRs show only significant $\mathrm{CO}(16-15)$ emission at very high densities and radiation fields $\left(n \sim 10^{6} \mathrm{~cm}^{-3}\right.$ and $\left.G_{0} \sim 10^{4}\right)$. This very dense and strongly irradiated gas, however, has a very small filling factor on large (galaxy) scales, and, the probability of observing a PDR with a very high $\mathrm{CO}(16-15)$ intensity is low.

The difference between the PDR and XDR models is seen even better by considering the intensity ratios of these high rotational transitions (see Fig. 6). A good example is the $\mathrm{CO}(16-$ $15) / \mathrm{CO}(1-0)$ ratio, which ranges from $10^{-3}$ to $10^{3}$ for PDRs, 

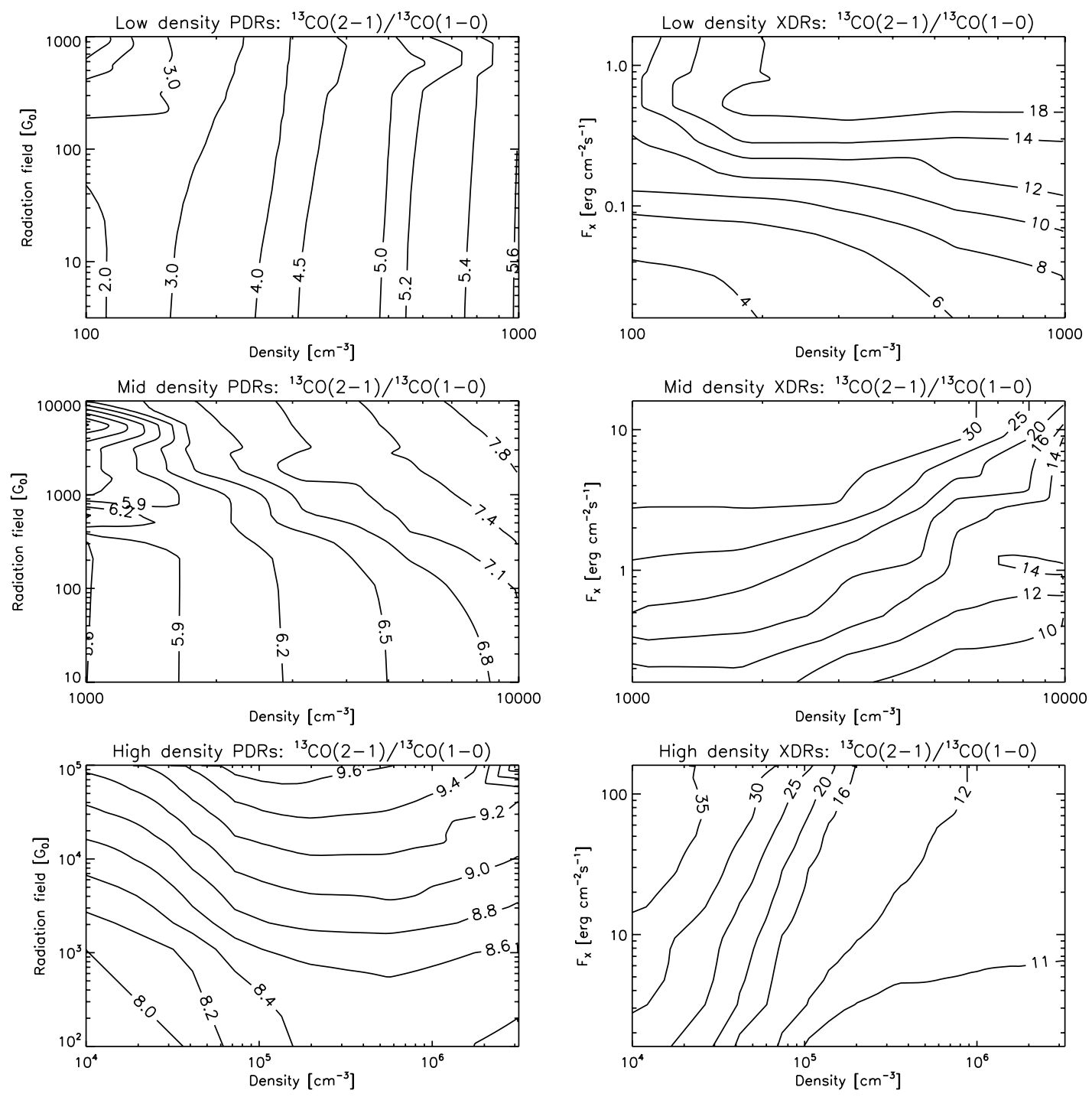

Fig. 7. ${ }^{13} \mathrm{CO}(2-1) /{ }^{13} \mathrm{CO}(1-0)$ ratio for PDR (left $)$ and XDR (right) models.

and from 10 to $>10^{4}$ for XDRs. Especially for densities between $10^{4}-10^{5} \mathrm{~cm}^{-3}$, it is very easy to distinguish PDRs from XDRs.

\section{3. ${ }^{13} \mathrm{CO}$ rotational lines}

The ${ }^{13} \mathrm{CO}$ lines have critical densities and upper state energies for the rotational transitions almost identical to those of ${ }^{12} \mathrm{CO}$. As we have adopted an abundance ratio ${ }^{12} \mathrm{C} /{ }^{13} \mathrm{C}=40$ in our models, ${ }^{13} \mathrm{CO}$ abundances are relatively low and the lines are much less optically thick.

The ${ }^{13} \mathrm{CO}(1-0)$ line intensities (Fig. A.6), show the same trends with density and radiation field as the $\mathrm{CO}(1-0)$ line, but there is a larger spread in intensity. For example, the difference in the $\mathrm{CO}(1-0)$ line emission in the low density PDRs (cloud type $\mathrm{C}$ ) is a factor of two and more than a factor of 3 for the ${ }^{13} \mathrm{CO}$ line. This effect is even larger for the XDRs. We find similar results for both the ${ }^{13} \mathrm{CO}(2-1)$ (not shown) and ${ }^{13} \mathrm{CO}(3-$ 2) line intensities as is evident from the corresponding ${ }^{13} \mathrm{CO}$ line ratios (see Fig. 7 and 8). A nice illustration of the $\mathrm{CO}$ and ${ }^{13} \mathrm{CO}$ behavior is supplied by the the $\mathrm{CO}(4-3) / \mathrm{CO}(1-0)$ versus the ${ }^{13} \mathrm{CO}(3-2) /{ }^{13} \mathrm{CO}(1-0)$ intensity ratio for the low density PDRs (cloud type C). The $\mathrm{CO}(4-3)$ line is pumped due to the fact that the lower rotational lines become optically thick. The
$\mathrm{CO}(4-3) / \mathrm{CO}(1-0)$ ratio changes only a factor of two, which is a factor two and a half for the ${ }^{13} \mathrm{CO}(3-2) /{ }^{13} \mathrm{CO}(1-0)$ ratio, despite the lower critical density of ${ }^{13} \mathrm{CO}(3-2)$.

\section{4. ${ }^{13} \mathrm{CO} / \mathrm{CO}$ ratios}

In Figs. 9 and A.8, the important isotopical intensity ratios of ${ }^{13} \mathrm{CO}(1-0) / \mathrm{CO}(1-0)$ and ${ }^{13} \mathrm{CO}(3-2) / \mathrm{CO}(3-2)$ are shown. Although for all cloud types (density regimes) the line intensities are larger in the XDR, the PDR isotopical ratios exceed those of XDRs, which means that the opacities of the XDR lines are larger as well.

\section{5. $[\mathrm{Cl}] 609 \mu \mathrm{m} /{ }^{13} \mathrm{CO}(2-1)$ ratio}

Figure 10 shows the $[\mathrm{CI}] 609 \mu \mathrm{m} /{ }^{13} \mathrm{CO}(2-1)$ ratios. For the same gas density and incident radiation field, PDRs have much lower ratios than XDRs. In the PDRs, the spread in the ratio is generally not very large. At low densities, ratios rapidly decrease from $72\left(n=10^{2} \mathrm{~cm}^{-3}\right)$ to $18\left(n=10^{3}\right)$ and then slowly fall off from $14.5\left(n=10^{4} \mathrm{~cm}^{-3}\right)$ to $2\left(n=10^{6} \mathrm{~cm}^{-3}\right)$. While the PDR ratios show a more or less steady decrease with density, a rather different picture is seen in XDRs. In each density range (cloud 

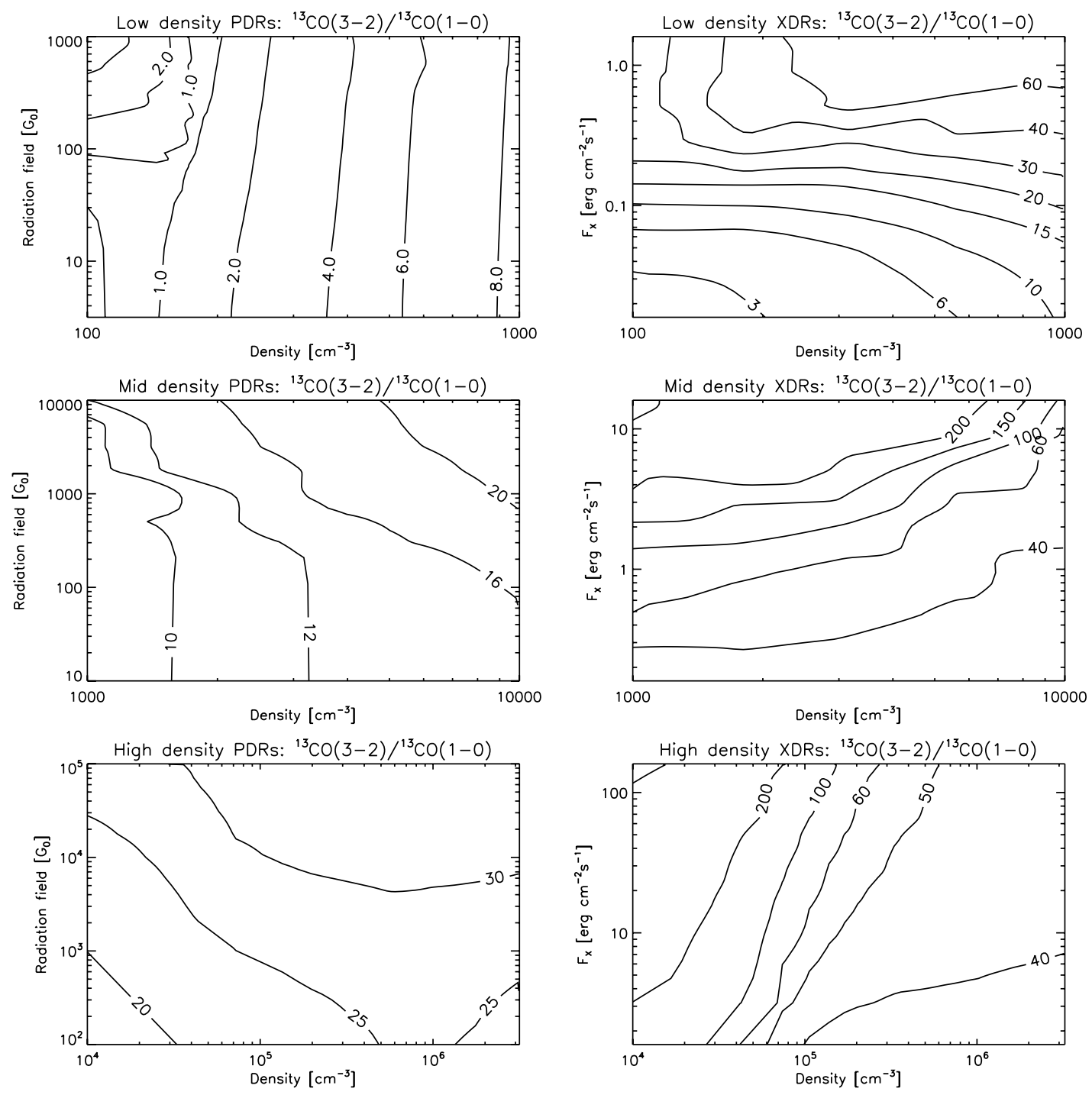

Fig. 8. ${ }^{13} \mathrm{CO}(3-2) /{ }^{13} \mathrm{CO}(1-0)$ ratio for PDR (left) and XDR (right) models.

type), the ratio changes by several orders of magnitude. For the lowest density in each cloud type, the column density is too low to attenuate the incident radiation field sufficiently to allow large amounts of $\mathrm{CO}$ to be present. On the other hand, neutral carbon occurs throughout the cloud, and, therefore, a large increase in the ratios are seen toward high $H_{\mathrm{X}} / n$.

\subsection{HCN rotational lines}

In Fig. A.9, we show the $\mathrm{HCN}(1-0)$ and $\mathrm{HCN}(4-3)$ line intensities for high density (cloud A type) PDR and XDR models only. Where the $\mathrm{CO}(4-3)$ line has a critical density of $n_{\mathrm{cr}} \sim 4 \times 10^{4} \mathrm{~cm}^{-3}$, the $\mathrm{HCN}(1-0)$ line has a critical density of $n_{\mathrm{cr}} \sim 3 \times 10^{6} \mathrm{~cm}^{-3}$. Higher rotational transitions such as $\mathrm{HCN}(2-1), n_{\mathrm{cr}} \sim 4 \times 10^{6} \mathrm{~cm}^{-3}$, and $\mathrm{HCN}(4-3), n_{\mathrm{cr}} \sim$ $2 \times 10^{7} \mathrm{~cm}^{-3}$, have even higher critical densities. The HCN rotational lines specifically trace the dense gas component in galaxies, and the line intensities from low and medium density gas are low. The $\mathrm{HCN}(1-0)$ line intensities range from $3 \times 10^{-11}$ to $2 \times 10^{-8} \mathrm{erg} \mathrm{s}^{-1} \mathrm{~cm}^{-2} \mathrm{sr}^{-1}$ for the low and mid density (type $\mathrm{C}$ en B) PDR models, and even less for the corresponding XDR models. Because of their poor observational prospects, we have limited ourselves to only showing the model results in the high density range (cloud type A). Typically, the $\operatorname{HCN}(1-0)$ emission is stronger in PDRs by a factor of about two for densities larger than $10^{5} \mathrm{~cm}^{-3}$. The $\mathrm{HCN}(4-3)$ diagrams show behavior very similar to that of $\mathrm{HCN}(1-0)$, but the PDR and XDR line strengths are now somewhat closer.

Typically, the HCN(1-0) emission is stronger in PDRs by a factor of about two for densities larger than $10^{5} \mathrm{~cm}^{-3}$. Our results are consistent with the chemical calculations of Lepp \& Dalgarno (1996) for different ionization rates, as follows. Our depth dependent models cause the HCN line emissivities to be the result of a line-of-sight integral over the HCN abundance pattern that results from a varying (attenuated) X-ray flux. Lepp \& Dalgarno (1996, their Fig. 3) find a rather narrow range of ionization rates for which the HCN abundance is high and consequently the XDR HCN line emissivities have difficulty competing with the PDR ones. The HCN(4-3) contour plots show about the same features as seen for the $\mathrm{HCN}(1-0)$. However, the PDR and XDR line strengths are now somewhat closer.

\subsection{HCN/CO line intensity ratios}

Intensity ratios of lines from the same species, such as the $\mathrm{CO}(4-3) / \mathrm{CO}(1-0)$ ratio, vary with column density, due to the 

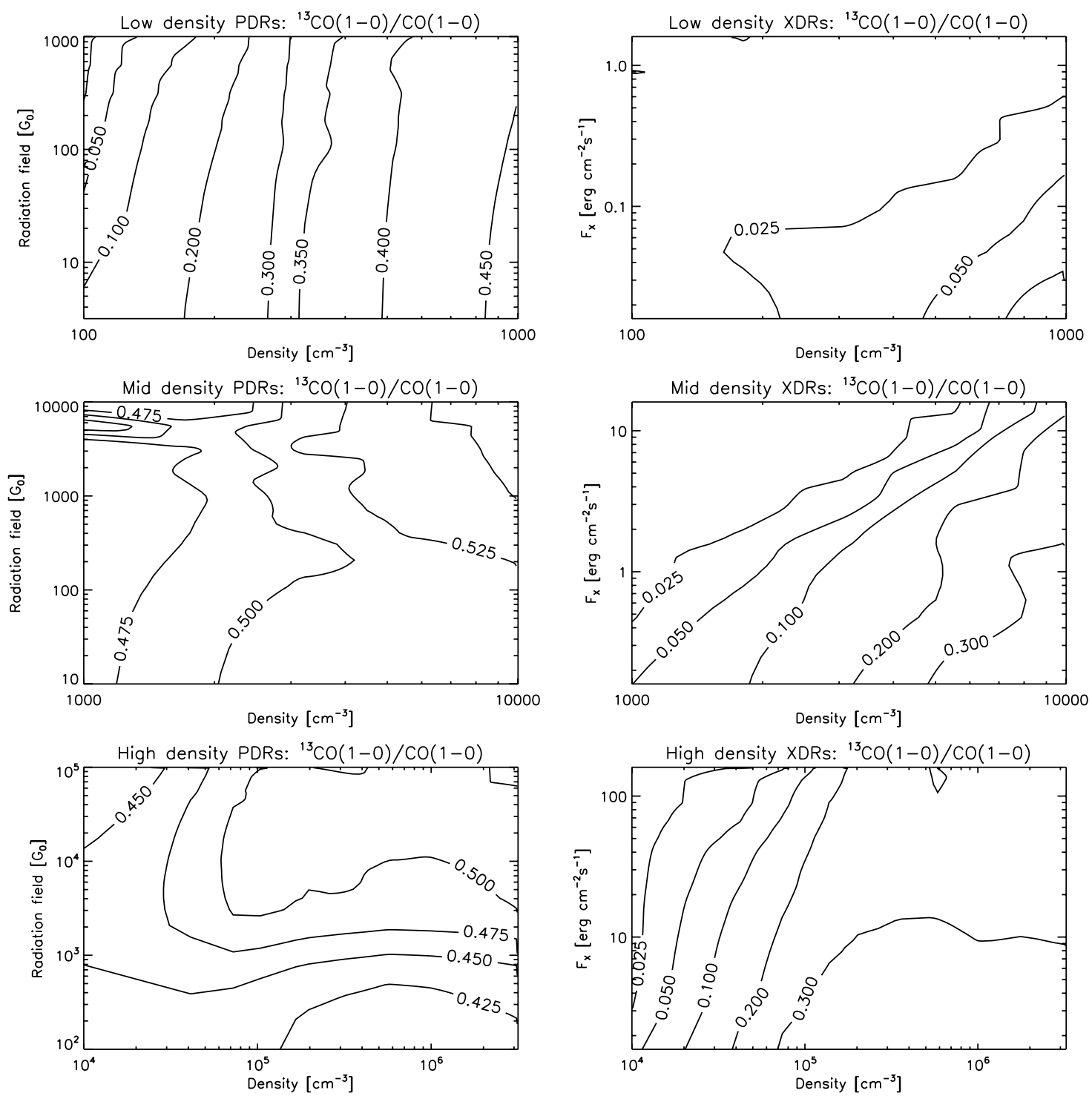

Fig. 9. ${ }^{13} \mathrm{CO}(1-0) / \mathrm{CO}(1-0)$ ratio for $\mathrm{PDR}($ left $)$ and $\mathrm{XDR}($ right $)$ models.

temperature gradient throughout the cloud, optical depth effects, varying abundance etc. Intensity ratios of lines from different species, in addition vary because of abundance ratio differences, complicating the interpretation of such line intensity ratios. In this section we turn our attention to the $\mathrm{HCN} / \mathrm{CO}$ ratio, and start by showing in Figs. 11 and A.10 the cumulative line intensity ratios for a set of PDRs and XDRs at densities ranging from $n=10^{4}-10^{6} \mathrm{~cm}^{-3}$ and incident fluxes between $G_{0}=10^{3}-10^{5}$ $\left(F_{\mathrm{X}}=1.6-160 \mathrm{erg} \mathrm{s}^{-1} \mathrm{~cm}^{-2}\right)$. The cumulative line intensity is the emergent intensity arising from the edge of the cloud to column density $N_{\mathrm{H}}=n_{\mathrm{H}} z$ :

$I(z)=\frac{1}{2 \pi} \int_{0}^{z} \Lambda\left(z^{\prime}\right) \mathrm{d} z^{\prime}$

In the PDR, both the $\mathrm{HCN}(1-0) / \mathrm{CO}(1-0)$ and the $\mathrm{HCN}(4-3) /$ $\mathrm{CO}(4-3)$ ratio show a minimum. The $\mathrm{HCN}$ abundance shows a drop around the $\mathrm{H} / \mathrm{H}_{2}$ transition, while $\mathrm{CO}$ has its maximum abundance beyond the $\mathrm{CO}$ transition. Deeper in the cloud, the $\mathrm{HCN} / \mathrm{CO}$ abundance ratio is more or less constant, but the $\mathrm{CO}$ line becomes optically thick and therefore the ratio increases.
When $H_{\mathrm{X}} / n$ is low in the XDR model, the gas is molecular, and the $\mathrm{HCN} / \mathrm{CO}$ abundance ratio is more or less constant. A slow rise in the ratio is seen, as the $\mathrm{CO}$ line becomes optically thick. When the outer part of the cloud is atomic (for high $\left.H_{\mathrm{X}} / n\right), \mathrm{HCN}$ shows a maximum, before the $\mathrm{H} / \mathrm{H}_{2}$ transition. This also produces a maximum in the $\mathrm{HCN}(1-0) / \mathrm{CO}(1-0)$ ratio. In the PDR, the variation is not that large for column densities $N_{\mathrm{H}}>10^{22} \mathrm{~cm}^{-2}$. In the XDRs, however, the variation between $N_{\mathrm{H}}=10^{22}$ and $10^{23} \mathrm{~cm}^{-2}$ can be rather large.

In Fig. 12, we show the $\mathrm{HCN}(1-0) / \mathrm{CO}(1-0)$ and $\mathrm{HCN}(4-3) /$ $\mathrm{CO}(4-3)$ line ratios for a fixed cloud size of one parsec (cloud type A). The variation in the ratios is relatively large, due to the high critical densities of the HCN transitions. The PDR models produce the highest $\mathrm{HCN}(1-0) / \mathrm{CO}(1-0)$ ratios, which are attained at large densities $\left(n>10^{6} \mathrm{~cm}^{-3}\right)$ and may exceed unity. The corresponding XDRs have ratios are only $0.1-0.2$. The interpretation of the low $\mathbf{J}$ transitions is very difficult due to high opacities, especially in the PDRs. For this reason, we also show the $\mathrm{HCN}(4-3) / \mathrm{CO}(4-3)$ ratio, which shows similar trends with density and radiation field, but with somewhat lower absolute ratios. 

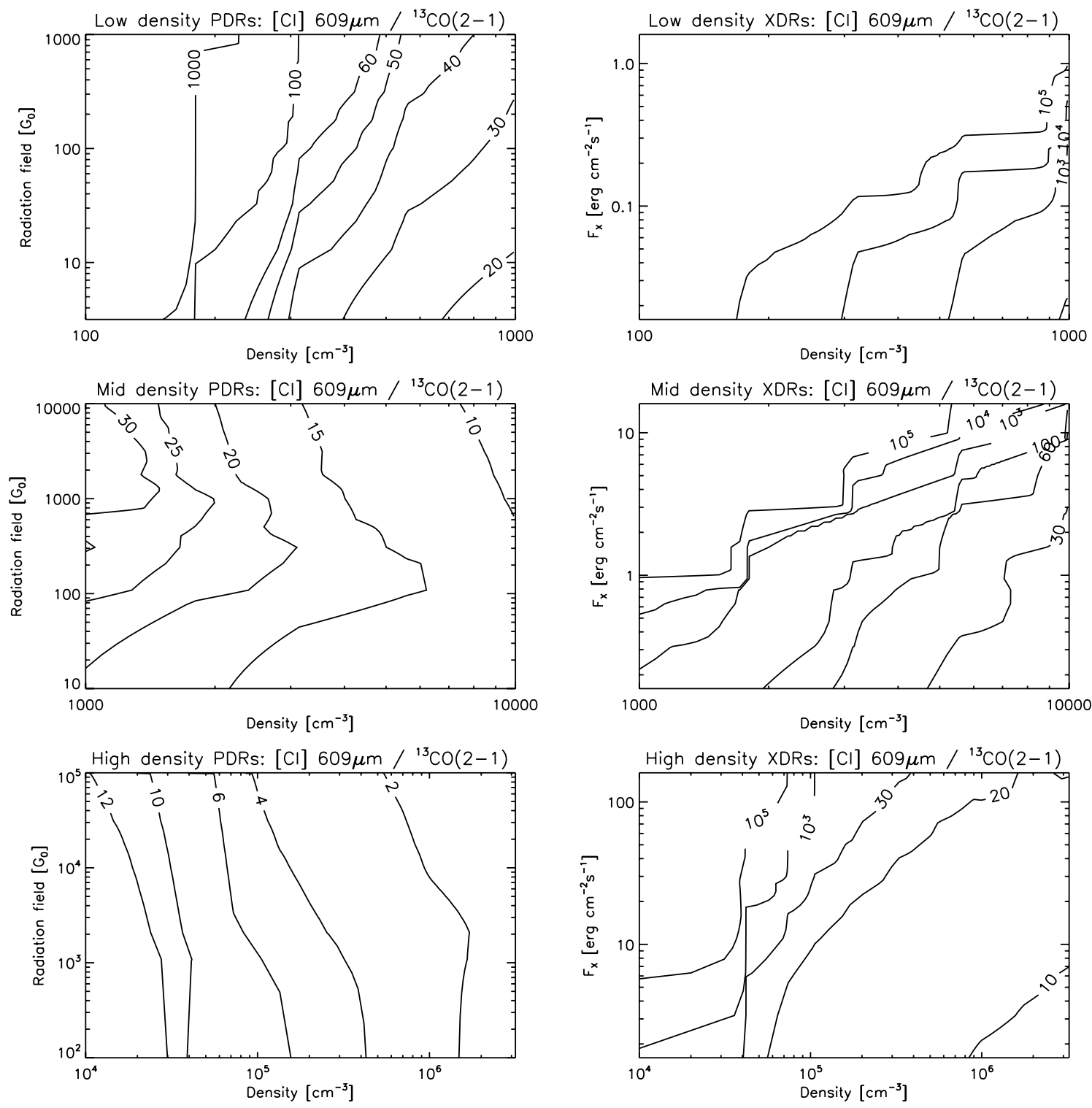

Fig. 10. CI $609 \mu \mathrm{m} /{ }^{13} \mathrm{CO}(2-1)$ ratio for PDR (left) and XDR (right) models.

\section{8. $\mathrm{HCO}^{+}$rotational lines and $\mathrm{HCN} / \mathrm{HCO}^{+}$line intensity ratios}

In Fig. A.11, we show the $\mathrm{HCO}^{+}(1-0)$ and $\mathrm{HCO}^{+}(4-3)$ line intensities, with critical densities $n_{\mathrm{cr}} \sim 2 \times 10^{5}$ and $4 \times 10^{6} \mathrm{~cm}^{-3}$, respectively. These critical densities are significantly lower than for $\mathrm{HCN}$, causing a smaller spread in line intensities. Typically, the $\mathrm{HCO}^{+}$lines are stronger in XDRs than in PDRs by a factor of at least three. This is a direct consequence of the higher ionization degree in XDRs (Meijerink \& Spaans 2005), leading to an enhanced $\mathrm{HCO}^{+}$formation rate.

Note in this that Lepp \& Dalgarno (1996, their Fig. 2) find a rather wide range of ionization rates for which the $\mathrm{HCO}^{+}$abundance is large. As for the HCN discussed above, this is consistent with our results since we integrate the depth dependent $\mathrm{HCO}^{+}$ abundance profile that results from the attenuation of the impinging X-ray flux. The $\mathrm{HCO}^{+}$line-of-sight integral thus picks up a large contribution and competes favorably with the PDR line emissivities.

In Figs. 13 and A.12, we show the cumulative $\mathrm{HCN}(1-0)$ / $\mathrm{HCO}^{+}(1-0)$ and $\mathrm{HCN}(4-3) / \mathrm{HCO}^{+}(4-3)$ line intensity ratios, for the same PDR and XDR models as in Sect. 5.5. Depending on the incident radiation field, $\mathrm{HCN}$ or $\mathrm{HCO}^{+}$is more abundant at the PDR edge of the cloud. Around the $\mathrm{H} / \mathrm{H}_{2}$ transition a minimum in the $\mathrm{HCO}^{+}$abundance is seen in the PDR. Deeper into the cloud, the $\mathrm{HCO}^{+}$abundance increases again, and is then constant. This is also the case for $\mathrm{HCN}$, and the $\mathrm{HCN} / \mathrm{HCO}^{+}$abundance ratio is larger than unity. Therefore, at sufficiently large columns and densities, the $\mathrm{HCN}(1-0) / \mathrm{HCO}^{+}(1-0)$ line intensity ratio becomes larger than one.

In the XDR models, $\mathrm{HCO}^{+}$is chemically less abundant than HCN for very large $H_{\mathrm{X}} / n$ (Meijerink \& Spaans 2005). For larger columns $\mathrm{HCO}^{+}$becomes more abundant, however, and eventually the cumulative column density of $\mathrm{HCO}^{+}$becomes larger than HCN (see specifically Fig. 10 in Paper I). This follows directly from the fact that the $\mathrm{HCO}^{+}$abundance is high over a much wider range of ionization rates than $\mathrm{HCN}$ (Lepp \& Dalgarno 1996, their Figs. 2 and 3).

Figure 14 clearly shows that the $\mathrm{HCN} / \mathrm{HCO}^{+}$ratio discriminates between PDRs and XDRs in the density range between $n=10^{5}$ and $10^{6.5} \mathrm{~cm}^{-3}$ (cloud type A). The HCN(1-0)/ $\mathrm{HCO}^{+}(1-0)$ and $\mathrm{HCN}(4-3) / \mathrm{HCO}^{+}(4-3)$ line ratios are both 

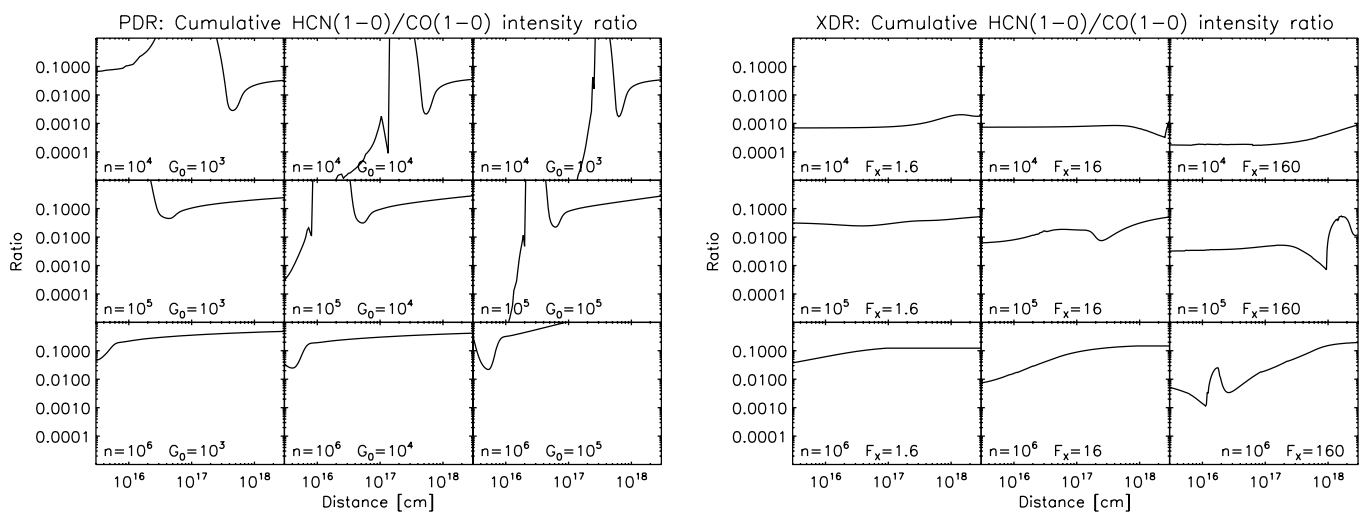

Fig. 11. Cumulative $\mathrm{HCN}(1-0) / \mathrm{CO}(1-0)$ line intensity ratios for PDR (left) and $\mathrm{XDR}($ right).
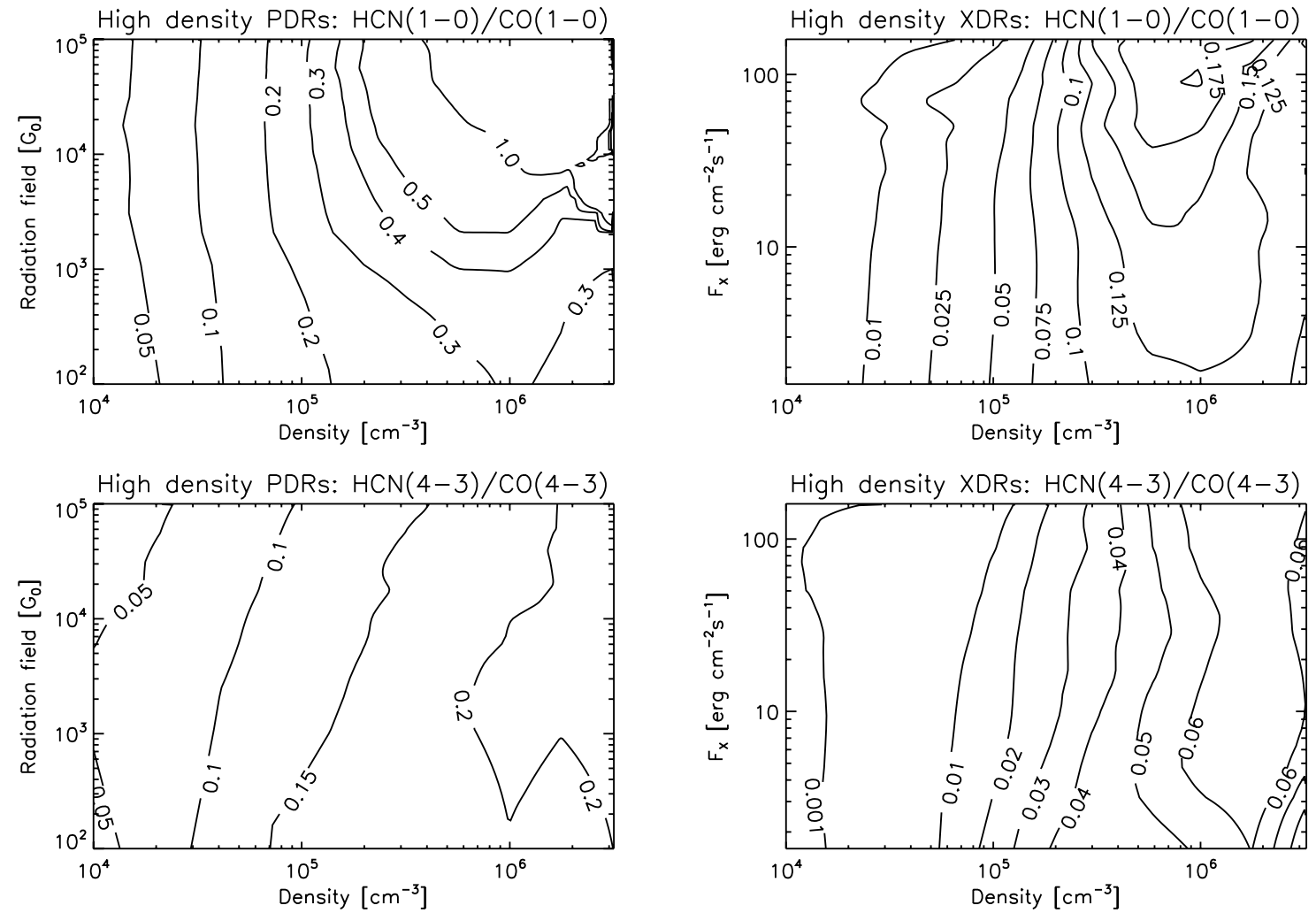

Fig. 12. $\mathrm{HCN}(1-0) / \mathrm{CO}(1-0)$ and $\mathrm{HCN}(4-3) / \mathrm{CO}(4-3)$ ratios for PDR (left) and XDR (right) models.
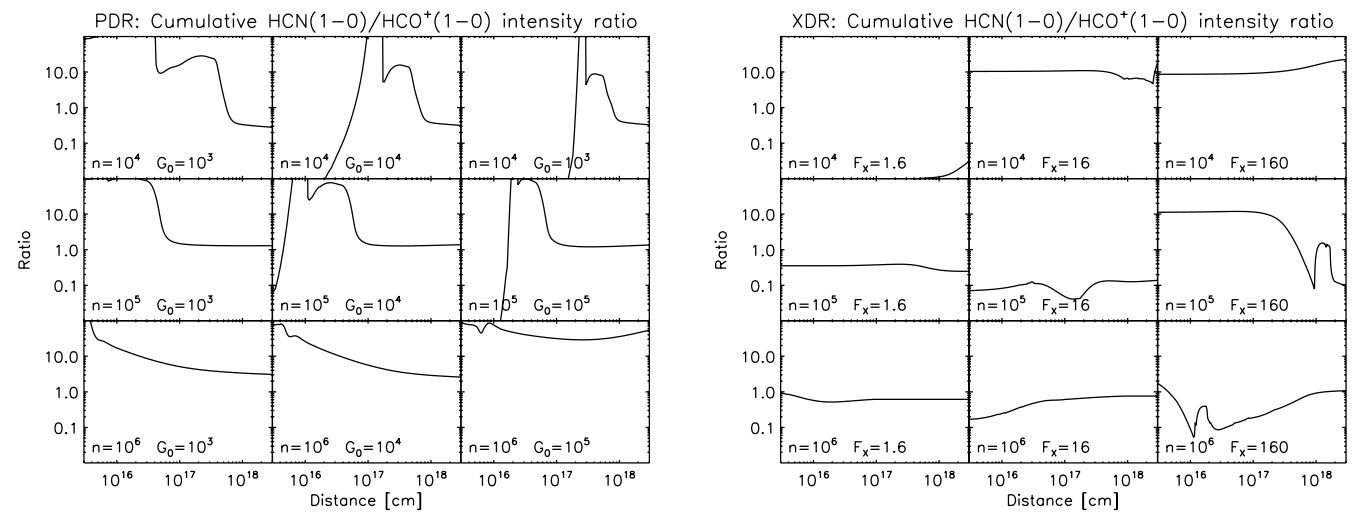

Fig. 13. Cumulative $\mathrm{HCN}(1-0) / \mathrm{HCO}^{+}(1-0)$ line intensity ratios for PDR (left) and XDR (right). 

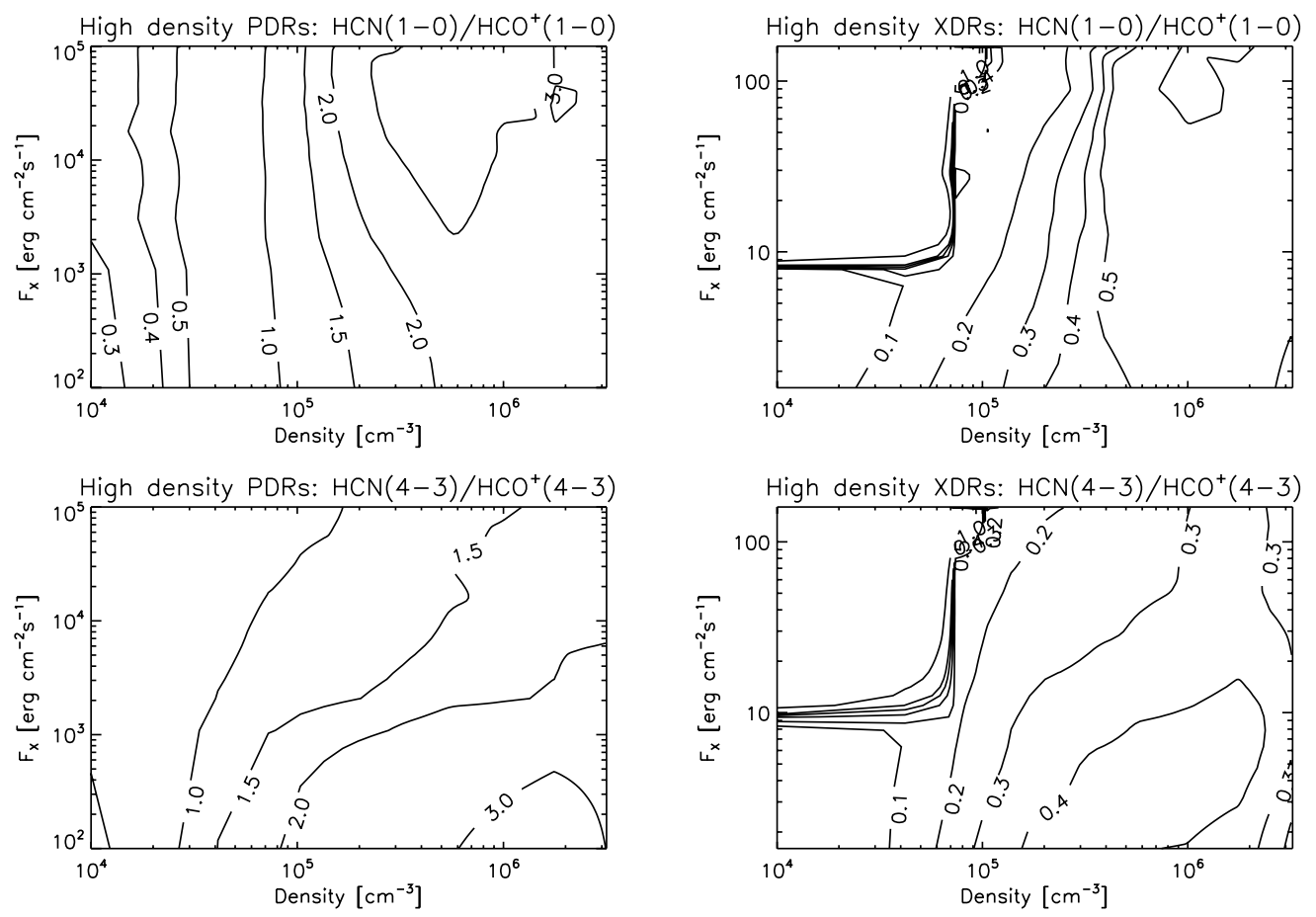

Fig. 14. $\mathrm{HCN}(1-0) / \mathrm{HCO}^{+}(1-0)$ and $\mathrm{HCN}(4-3) / \mathrm{HCO}^{+}(4-3)$ ratios for $\mathrm{PDR}($ left $)$ and $\mathrm{XDR}($ right $)$ models.
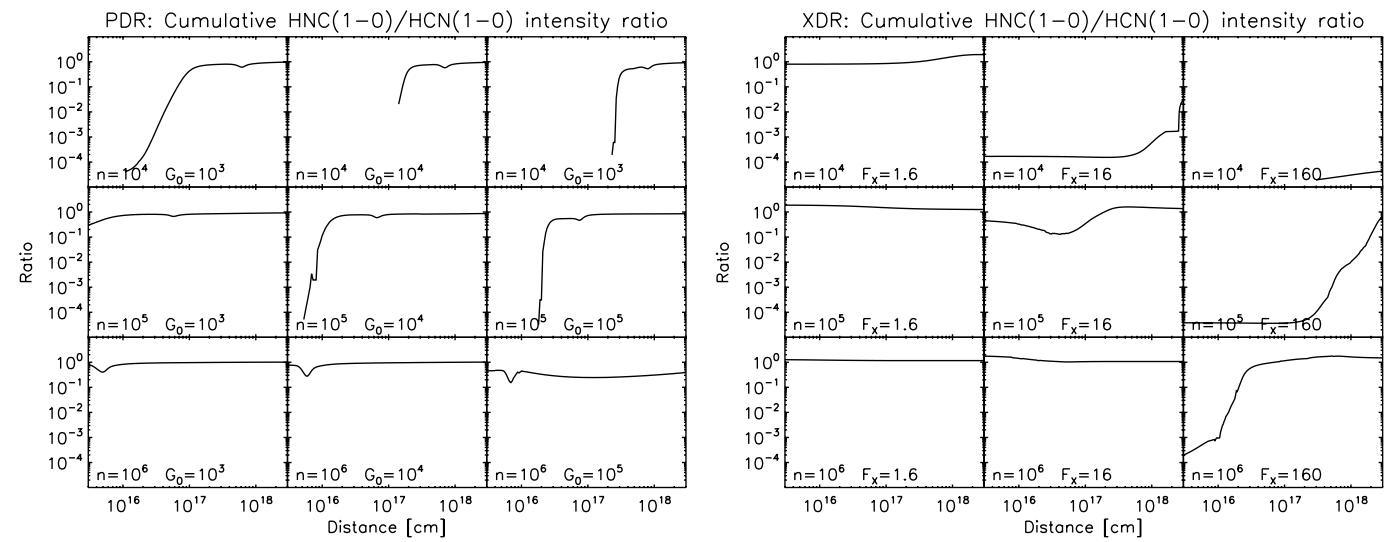

Fig. 15. Cumulative $\mathrm{HNC}(1-0) / \mathrm{HCN}(1-0)$ line intensity ratios for PDR (left) and XDR (right).

much larger in the PDR models than XDR models, for columns of $10^{23} \mathrm{~cm}^{-2}$ and larger (Paper I). The difference ranges between a factor of 4-10, depending on the density. The XDR HCN(1-0)/ $\mathrm{HCO}^{+}(1-0)$ ratio becomes larger than unity for more modest columns of $10^{22.5} \mathrm{~cm}^{-2}$ and less.

\section{9. $\mathrm{HNC/HCN}$ ratios}

The critical densities of HCN and HNC are almost identical, so that the only differences in line ratio should be due to differences in the abundances. In PDRs, HCN is more abundant in the radical region, but deeper in the cloud the abundance ratio approaches unity. In XDRs, HCN is more abundant in the highly ionized part of the cloud. However, HNC is equally or even more abundant than HCN deep into the cloud. As a result, the $\mathrm{HNC}(1-0) / \mathrm{HCN}(1-0)$ line intensity ratio is around one for the PDRs if the column density is larger than $10^{22} \mathrm{~cm}^{-2}$, while the $\mathrm{HNC}(1-0) / \mathrm{HCN}(1-0)$ ratio is less than unity for $N_{\mathrm{H}}<$ $10^{22} \mathrm{~cm}^{-2}$. The XDR models, however, show low ratios for the low, $\sim 10^{4} \mathrm{~cm}^{-3}$, densities and strong, $>10 \mathrm{erg} \mathrm{s}^{-1} \mathrm{~cm}^{-2}$, radiation fields. The ratios increase for lower incident radiation fields, and at highest densities $\left(n=10^{6.5} \mathrm{~cm}^{-3}\right)$ the line ratios are always larger than one, irrespective of irradiation.

In the PDRs, the $\mathrm{HNC}(4-3) / \mathrm{HCN}(4-3)$ ratio quickly drops below unity at densities below $n=10^{5} \mathrm{~cm}^{-3}$. This density is far below the critical densities of the lines, and therefore high temperatures are needed to excite them. Such high temperatures are indeed found in the radical regions of the PDRs, but there the HNC abundance is much lower than the HCN abundance, which explains the drop in the ratio. In the XDRs, the HNC(4$3) / \mathrm{HCN}(4-3)$ ratios are quite similar to the $\mathrm{HNC}(1-0) / \mathrm{HCN}(1-$ 0 ) ratios, except for densities $n>10^{6} \mathrm{~cm}^{-3}$, where they are even high than these.

\subsection{SiO and CS}

Although $\mathrm{SiO}$ is usually considered to be a good tracer of shocks, we do find that the $\mathrm{SiO}(1-0) / \mathrm{CO}(1-0)$ ratio is typically larger 

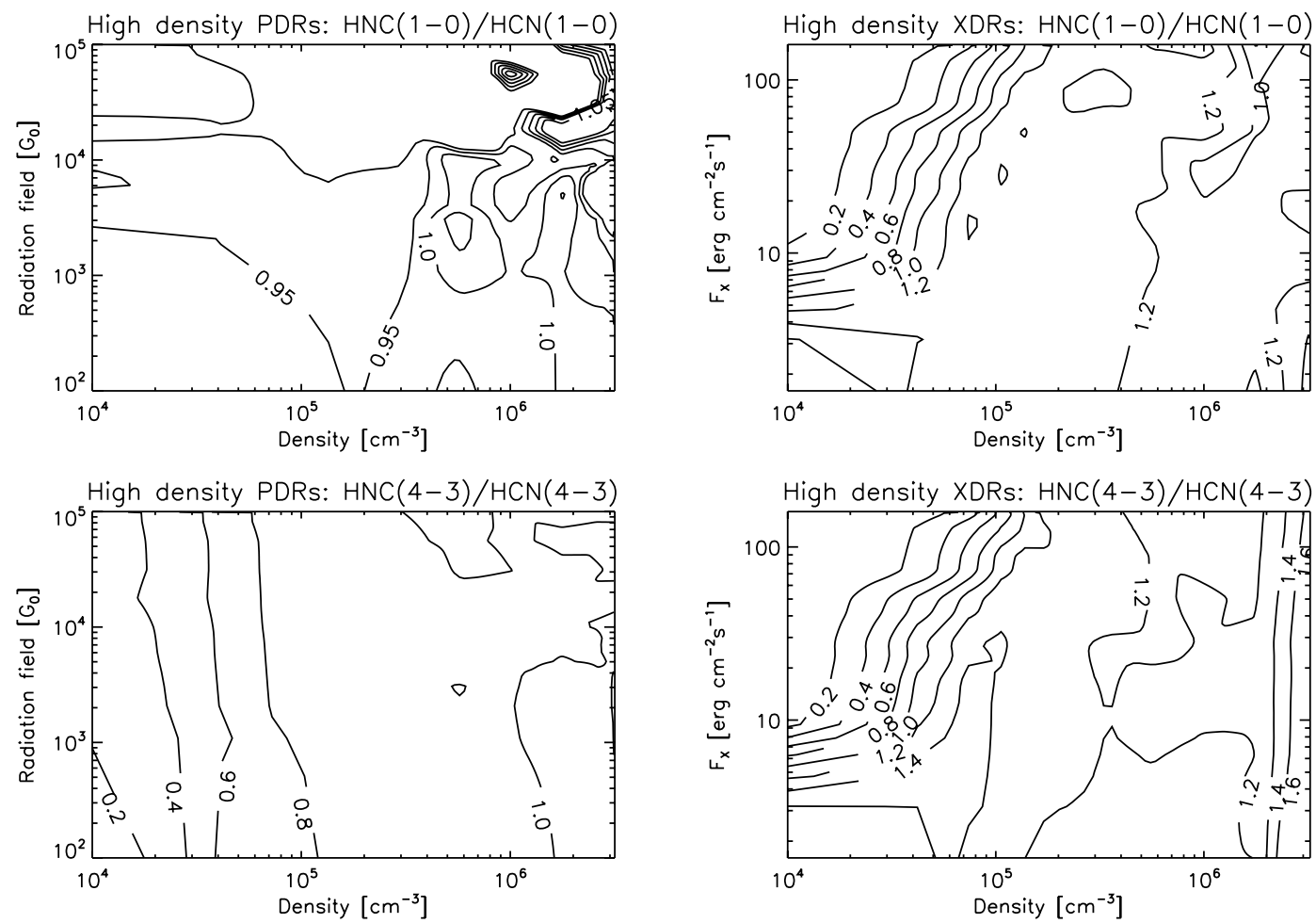

Fig. 16. $\mathrm{HNC}(1-0) / \mathrm{HCN}$ and $\mathrm{HNC}(4-3) / \mathrm{HCN}(4-3)$ ratios for PDR (left) and $\mathrm{XDR}$ (right) models.
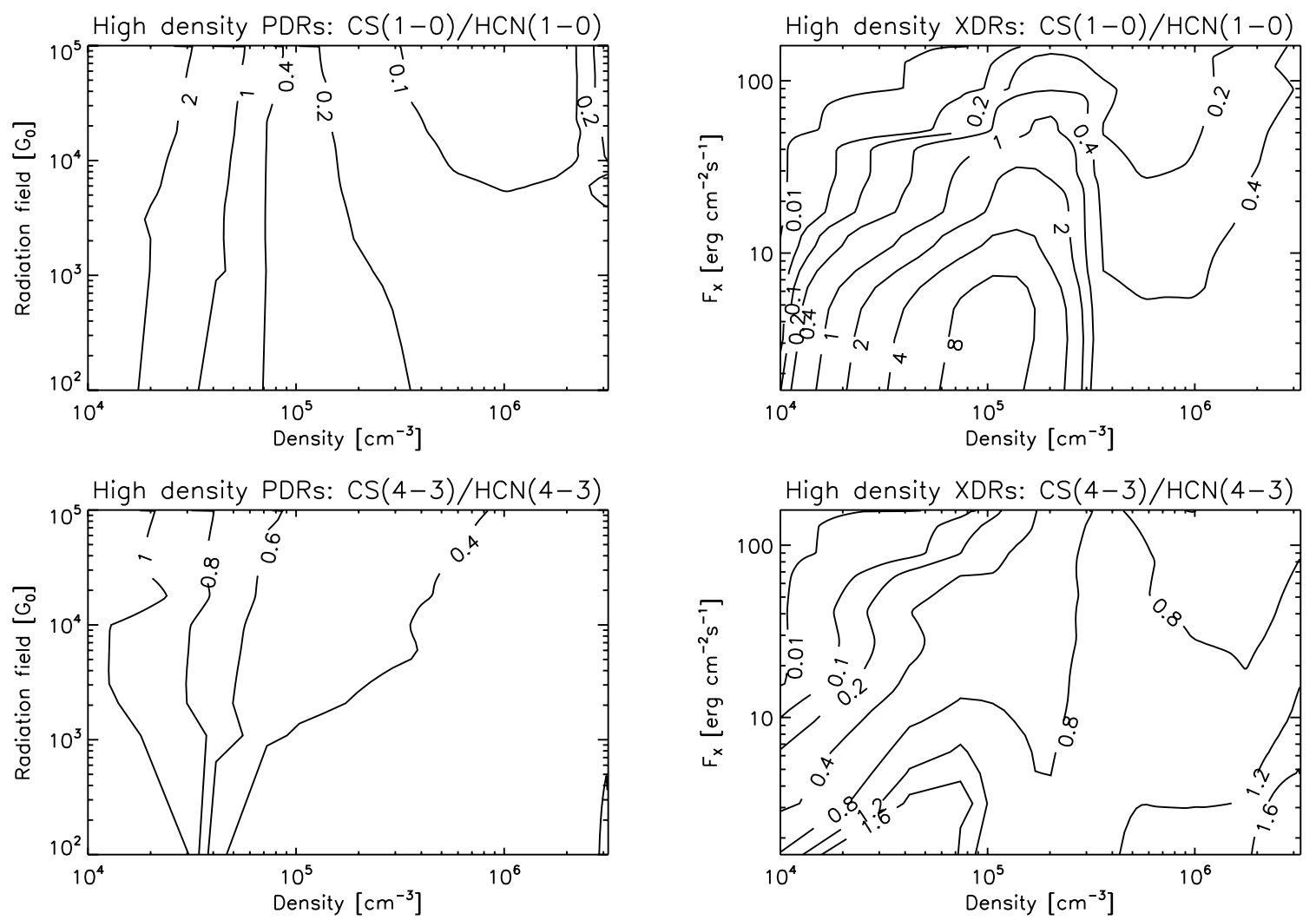

Fig. 17. $\mathrm{CS}(1-0) / \mathrm{HCN}$ and $\mathrm{CS}(4-3) / \mathrm{HCN}(4-3)$ ratios for PDR (left) and XDR (right) models.

in XDRs than in PDRs by a factor of $2-3$, for densities around $10^{5.5} \mathrm{~cm}^{-3}$. For the higher excitation $J=4-3$ lines, the effect disappears because $\mathrm{CO}$ is generally warmer in XDRs compared to PDRs. 

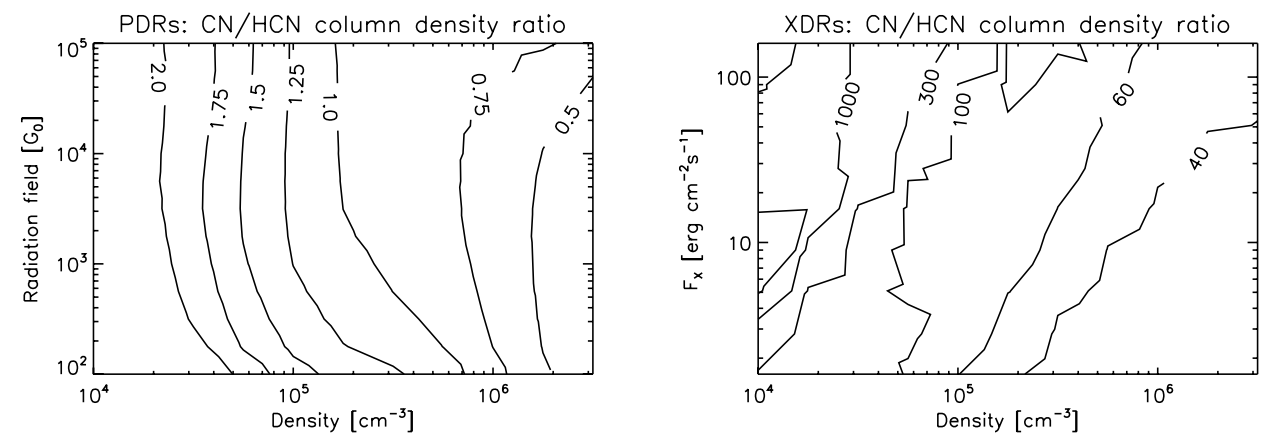

Fig. 18. CN/HCN column density ratios for PDR (left) and XDR (right) models.
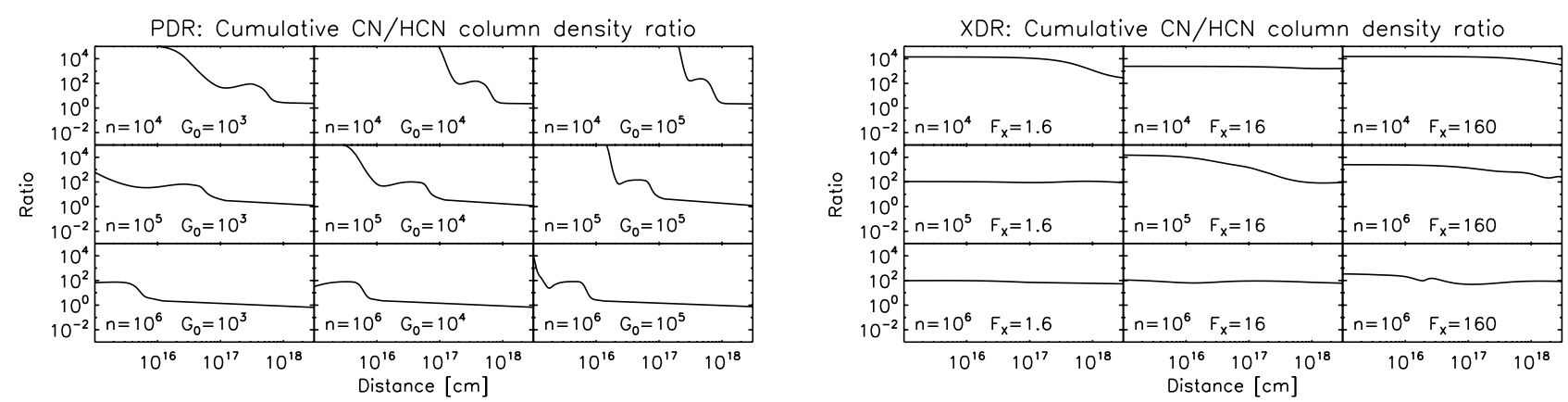

Fig. 19. Cumulative $\mathrm{CN} / \mathrm{HCN}$ column density ratios for PDR (left) and XDR (right) models.

The $\mathrm{CS}(1-0) / \mathrm{HCN}(1-0)$ ratio is a factor of two larger (smaller) in XDRs for densities above (below) $10^{5} \mathrm{~cm}^{-3}$. Interestingly, the corresponding 4-3 ratio continues this trend but changes in the ratio from $10^{4}$ to $10^{6} \mathrm{~cm}^{-3}$ are now as large as a factor of 10 .

\section{Column density ratios}

Unfortunately, for many molecular species of interest no reliable collisional cross sections are available. For these species we are unable to accurately predict line intensities, but we can still calculate the column density ratios. In this section, we discuss column density ratios for a number of species that are of potential interest in attempts to discriminate between PDRs and XDRs.

\section{1. $\mathrm{CN} / \mathrm{HCN}$ column density ratio}

In Fig. 18, we show the $\mathrm{CN} / \mathrm{HCN}$ column density ratios. There is an enormous difference between the ratios for PDRs and XDRs. In the PDRs, the cloud type A ratios range from 0.5 $\left(n \sim 10^{6} \mathrm{~cm}^{-3}\right)$ to $2.0\left(n \sim 10^{4} \mathrm{~cm}^{-3}\right)$, while in the XDR models the same ratio varies from $40\left(n \sim 10^{6} \mathrm{~cm}^{-3}\right)$ to over a 1000 $\left(n \sim 10^{4} \mathrm{~cm}^{-3}\right)$. We find higher $\mathrm{CN} / \mathrm{HCN}$ ratios at lower densities, where the chemical rates are lower, making it more difficult to form large molecules. The PDR ratios are only dependent on density, which is explained by the fact that most $\mathrm{CN}$ and $\mathrm{HCN}$ molecules are formed beyond the $\mathrm{H} / \mathrm{H}_{2}$ transition. This part of the cloud is shielded from FUV photons and the chemistry is dominated by the cosmic ray ionization rate, which is the same in every model. In the XDR models, there is much less variation in the $\mathrm{CN}$ and $\mathrm{HCN}$ abundance throughout the cloud. The variations do not exceed more than two to three orders of magnitude, while this is over ten orders of magnitude in the PDR models. All parts of the cloud contribute almost equally to the column density ratio, including the region with very high $H_{\mathrm{X}} / n . H_{\mathrm{X}} / n$ is a major factor in the resulting ratio, and therefore the XDRs also show a large dependence on incident radiation field.

In Fig. 19, we show the PDR and XDR cumulative CN/HCN ratios for a few specific densities and radiation fields. The variation in the cumulative column density ratio is much less in the XDR models than in the PDR models. At PDR edges, the gas is highly ionized (as in the XDRs), and here we find ratios resembling those of XDRs. Abundances, however, are very low here because of the high photo-dissociation rate.

\section{2. $\mathrm{CH} / \mathrm{HCN}$ column density ratios}

In Fig. A.17, we show the $\mathrm{CH} / \mathrm{HCN}$ column density ratio. The differences between the ratios in PDRs and XDRs are even larger than in the case of $\mathrm{CN} / \mathrm{HCN}$. While the PDR ratios increase from $0.2\left(n \sim 10^{6} \mathrm{~cm}^{-3}\right)$ to $0.9\left(n \sim 10^{4} \mathrm{~cm}^{-3}\right)$, the XDR ratios range from 20 to more than 10000 . The PDR ratio does not depend on density only. At relatively low densities $\left(n=1-3 \times 10^{4} \mathrm{~cm}^{-3}\right)$, we find a dependency on incident radiation field as well. $\mathrm{CH}$ reaches its maximum abundance at greater depths than $\mathrm{HCN}$, which means that the cumulative ratio is still increasing at large column densities. Our model cloud sizes are not large enough to allow the ratio to converge to a constant ratio (see Fig. A.18). For the XDR models, we can roughly state that the ratio increases toward higher $H_{\mathrm{X}} / n$. However, at $n \sim 10^{5} \mathrm{~cm}^{-3}$ the lowest ratio is not found at the lowest incident radiation field strength. At such densities, the limited cloud sizes are comparable to the depth of the $\mathrm{H} / \mathrm{H}_{2}$ transition. This transition is included in models with the lowest radiation field strengths, but not in those with the highest radiation field strengths. 


\section{3. $\mathrm{CH}^{+} / \mathrm{HCN}$ column density ratios}

In Fig. A.19, we show the $\mathrm{CH}^{+} / \mathrm{HCN}$ ratios for both PDR and XDR models. They range from $10^{-6}\left(n \sim 10^{6} \mathrm{~cm}^{-3}\right)$ to $6 \times 10^{-4}$ $\left(n \sim 10^{4} \mathrm{~cm}^{-3}\right)$ in the PDRs, and from $10^{-3}\left(n \sim 10^{6} \mathrm{~cm}^{-3}\right)$ to over $1000\left(n \sim 10^{4} \mathrm{~cm}^{-3}\right)$ in the XDRs. In the PDR models, the highest $\mathrm{CH}^{+}$abundance is seen close to the edge of the cloud but it decreases very quickly beyond the $\mathrm{H} / \mathrm{H}_{2}$ transition. $\mathrm{HCN}$, on the contrary, reaches its highest abundance beyond this transition. This explains the decrease of the PDR cumulative column density ratios with increasing depth (Fig. A.20). The PDR models show a dependence on both density and incident radiation field for this ratio. The decrease in the ratio with increasing density is caused by the fact that on the one hand $\mathrm{CH}^{+}$is more easily destroyed (due to higher recombination rates) and on the other hand HCN more easily formed when densities are higher. The ratios do not converge to a constant value deep into the cloud. At identical densities, the ratio increases with increasing radiation field, since the HCN column densities become smaller while the $\mathrm{CH}^{+}$column densities become larger for the cloudsize considered here. In the XDR models, the largest ratios are seen for the highest $H_{\mathrm{X}} / n$ (low density and high incident radiation field). The fluctuations in the $\mathrm{CH}^{+}$and $\mathrm{HCN}$ abundances are more gradual. The $\mathrm{HCN}$ abundance increases and the $\mathrm{CH}^{+}$ abundance decreases when the X-ray photons are gradually absorbed. Therefore, the XDR cumulative column densities ratios show less variation than the PDR ratios (see Fig. A.20).

\section{4. $\mathrm{HCO} / \mathrm{HCO}^{+}$column density ratios}

Figure A.21 shows the $\mathrm{HCO} / \mathrm{HCO}^{+}$column density ratios. For the PDRs, we find much larger ratios than for the XDRs. The PDRs show ratios between $0.1\left(n \sim 10^{4} \mathrm{~cm}^{-3}\right)$ and 10.0 $\left(n \sim 10^{6} \mathrm{~cm}^{-3}\right)$, while the ratios in the XDR range from $10^{-5}$ $\left(n \sim 10^{4} \mathrm{~cm}^{-3}\right)$ to $10^{-3}\left(n \sim 10^{6} \mathrm{~cm}^{-3}\right)$. In the PDRs, the ratios depend only on density for radiation fields $G_{0}<10^{4}$. With larger incident radiation fields, the ratio becomes dependent on the radiation field strength as well. The HCO abundance reaches its maximum and more or less constant abundance somewhat deeper into the cloud than $\mathrm{HCO}^{+}$. As column density increases, the $\mathrm{HCO} / \mathrm{HCO}^{+}$ratio slowly converges to a constant value (see Fig. A.22). For large radiation fields $\left(G_{0}>10^{4}\right)$, the cloud size considered here is too small to allow column densities to converge to a constant ratio. In the XDR models, the ratio depends on both density and radiation field in all regimes. The lowest ratios are seen for high $H_{\mathrm{X}} / n\left(n=10^{4} \mathrm{~cm}^{-3}\right.$ and $\left.F_{\mathrm{X}}=160\right)$.

\section{5. $\mathrm{HOC}^{+} / \mathrm{HCO}^{+}$column density ratios}

In Fig. A.23, we show the $\mathrm{HOC}^{+} / \mathrm{HCO}^{+}$column density ratios. The XDRs show larger ratios, ranging from $10^{-4}\left(n \sim 10^{6} \mathrm{~cm}^{-3}\right)$ to $0.6\left(n \sim 10^{4} \mathrm{~cm}^{-3}\right)$, than PDRs, where the ratios range from $10^{-7}$ to $10^{-5}$. The XDR ratios increase for larger $H_{\mathrm{X}} / n$ (with maximum at $n \sim 10^{4} \mathrm{~cm}^{-3}$ and $F_{\mathrm{X}}=160$ ). The behavior is more complex for the PDR models. The ratios depend on density only for $G_{0}>10^{4}$, but below this value there is also a dependency on radiation field strength. This is explained by the fact that ratios drop very fast, when the gas becomes molecular (Fig. A.24), but abundances are still significant. A large fraction of the gas at the edge of the cloud is molecular for densities $n>10^{5.5} \mathrm{~cm}^{-3}$ and radiation fields $G_{0}<10^{4}$. Here we find a fast decrease in the ratio for lower radiation fields.

\subsection{NO/CO column density ratios}

In Fig A.25, we show the NO/CO column density ratios. The ratios in XDRs are much larger, $10^{-4}\left(n \sim 10^{6} \mathrm{~cm}^{-3}\right)$ to $10^{-3}$ $\left(n \sim 10^{4} \mathrm{~cm}^{-3}\right)$, than for PDRs $\left(10^{-6}-10^{-5}\right)$. In the PDR the ratios are largely determined in the molecular part of the cloud and depend more or less on density only. The ratios in the XDR depend mostly on $H_{\mathrm{X}} / n$. The largest ratios are seen for the largest $H_{\mathrm{X}} / n\left(n \sim 10^{4} \mathrm{~cm}^{-3}\right.$ and $\left.F_{\mathrm{X}}=160\right)$.

$\mathrm{CO}(1-0)$ lines have optical depths of $\tau(\mathrm{CO}(1-0)) \sim 100$. Therefore, it could be possible to observe $\mathrm{NO}(1-0) / \mathrm{CO}(1-0)$ line intensity ratios as large as 0.1 , while the maximum column density ratios are only $10^{-3}$.

\section{7. $\mathrm{N}_{2} \mathrm{H}^{+} / \mathrm{CO}$ column density ratios}

In Fig. A.27, we show the $\mathrm{N}_{2} \mathrm{H}^{+} / \mathrm{CO}$ column density ratio. In both PDRs and XDRs the model ratios are rather low. They decrease with density in the PDR $\left(10^{-7}-10^{-9}\right)$, which is opposite to the behavior in XDRs. In the XDRs, we find a decrease with increasing $H_{\mathrm{X}} / n$. There are collisional data available for $\mathrm{N}_{2} \mathrm{H}^{+}$, but since the abundances are so low, the line intensities are too small to be observable.

\section{Summary and outlook}

We have presented a large set of PDR and XDR models that can be used to determine the physical conditions that pertain to irradiated gas clouds. This grid spans a large range in densities $\left(n_{\mathrm{H}}=10^{2}-10^{6.5} \mathrm{~cm}^{-3}\right)$, irradiation $\left(G_{0}=10^{0.5}-10^{5}\right.$ and $\left.F_{\mathrm{X}}=1.6 \times 10^{-2}-160 \mathrm{erg} \mathrm{cm}^{-2} \mathrm{~s}^{-1}\right)$ and column densities $\left(N_{\mathrm{H}}=1.5 \times 10^{22}-1 \times 10^{25} \mathrm{~cm}^{-2}\right)$. We have used the results to make predictions for the intensities and ratios of the most important atomic fine-structure lines, e.g., [CII], [OI], [CI], [SiII], and [FeII], rotational lines for molecular species such as $\mathrm{HCO}^{+}, \mathrm{HCN}, \mathrm{HNC}, \mathrm{CS}$ and $\mathrm{SiO}$ (up to $J=4$ ), $\mathrm{CO}$ and ${ }^{13} \mathrm{CO}$ up to $J=16$, and for column densities for $\mathrm{CN}, \mathrm{CH}, \mathrm{CH}^{+}, \mathrm{HCO}$, $\mathrm{HOC}^{+}, \mathrm{NO}$, and $\mathrm{N}_{2} \mathrm{H}^{+}$. It is not possible to to present all the results, but they are available on-line at the following URL: http://www.strw. leidenuniv.nl/ mei jerin/grid/

Here we summarize the most important conclusions:

1. The surface temperatures are higher (lower) in PDRs compared to XDRs for densities $n>10^{4} \mathrm{~cm}^{-3}\left(n<10^{4} \mathrm{~cm}^{-3}\right)$. Two opposing effects play a major role in determining the resulting surface temperature: (1) The heating efficiency, which is much higher in XDRs (up to 70 percent) than in PDRs (0.5-3.0 percent); (2) The absorption cross sections which are much smaller for X-rays than for FUV photons.

2. For the atomic lines, we find that the fine-structure line ratios of [SiII] $35 \mu \mathrm{m} /[\mathrm{CII}] 158 \mu \mathrm{m}$, [OI] $63 \mu \mathrm{m} /[\mathrm{CII}] 158 \mu \mathrm{m}$, [FeII] $26 \mu \mathrm{m} /[\mathrm{CII}] 158 \mu \mathrm{m}$, and [CI] $369 \mu \mathrm{m} /[\mathrm{CI}] 609 \mu \mathrm{m}$ are higher in XDRs than in PDRs, for a given density, column, and irradiation strength. Whereas PDR ratios depend on density and irradiation strength only, XDR depend on column density as well. In PDRs, fine-structure line emission is only produced at the edge of the cloud, while in XDRs almost all parts of the cloud contribute.

3. We find higher $\mathrm{CO}$ line ratios for XDRs. In PDRs, CO is formed beyond the $\mathrm{H} / \mathrm{H}_{2}$ transition and typically has temperatures in the range $T \sim 20-50 \mathrm{~K}$. In XDRs, CO is present throughout the cloud in significant abundances, even in the highly ionized part. When using CO line ratios, the best way 
to distinguish between PDRs and XDRs is to consider ratios such as $\mathrm{CO}(16-15) / \mathrm{CO}(1-0)$, where the differences are largest (Fig. 6).

4. $\mathrm{HCN} / \mathrm{HCO}^{+}$ratios discriminate well between PDRs and XDRs, even in the lower rotational lines, and especially when densities are as high as $n>10^{5} \mathrm{~cm}^{-3}$. At such densities, the $\mathrm{HCN}(1-0) / \mathrm{HCO}^{+}(1-0)$ line intensity ratios are $<1$ in XDRs, while PDRs have ratios $>1$ for column densities $N_{\mathrm{H}}>10^{23} \mathrm{~cm}^{-2}$. Although the $\mathrm{HCN} / \mathrm{HCO}^{+}$line ratio in an XDR may become even larger in clouds of relatively modest density $\left(10^{4} \mathrm{~cm}^{-3}\right)$ subjected to high radiation field strengths $\left(>100 \mathrm{erg} \mathrm{s}^{-1} \mathrm{~cm}^{-2}\right.$ ), we find that the line intensities in this part of parameter space are too low to be detectable (see also Meijerink et al. 2006).

5. For densities between $10^{4}$ and $10^{6.5} \mathrm{~cm}^{-3}, \mathrm{HNC}(1-$ $0) / \mathrm{HCN}(1-0)$ ratios in PDRs are of order $1(<1)$ for columns larger (smaller) than $10^{22} \mathrm{~cm}^{-2}$, while the ratios range between 0.2-1.2 for XDRs. PDR HNC(4-3)/HCN(4-3) ratios never exceed unity, while we find $\mathrm{XDR}$ ratios up to 1.6 for densities $n>10^{6} \mathrm{~cm}^{-3}$.

6. $\mathrm{HCN} / \mathrm{CO}$ ratios are typically smaller for in XDRs than in PDRs, for two reasons: (1) The HCN abundance is boosted only in high (column) density gas, with columns in excess of $10^{23} \mathrm{~cm}^{-2}$ and densities larger than $10^{4} \mathrm{~cm}^{-2}$; (2) CO is warmer in XDRs, which leads to stronger emission, and this suppresses the $\mathrm{HCN} / \mathrm{CO}$ ratios as well. In PDRs, the very high $\mathrm{HCN}(1-0) / \mathrm{CO}(1-0)$ ratios of order $>1$ are only obtained at very high column densities $\left(N_{\mathrm{H}}>10^{23} \mathrm{~cm}^{-2}\right)$. High column densities $N_{\mathrm{H}}$ are needed to obtain a large $\mathrm{HCN}$ column density and to obtain optically thick HCN lines. This happens much faster for $\mathrm{CO}$, due to its higher fractional abundance.

7. We find that $\mathrm{CN} / \mathrm{HCN}, \mathrm{NO} / \mathrm{CO}$, and $\mathrm{HOC}^{+} / \mathrm{HCO}^{+}$column density ratios are discriminant between PDRs and XDRs. Molecules such as $\mathrm{CH}$ and $\mathrm{CH}^{+}$also look very promising. However, we urgently need reliable collisional cross section data in order to make predictions for observed line ratios.

We conclude that both atomic fine-structure and molecular rotational lines have significant diagnostic value to allow us to distinguish between clouds irradiated by star-bursts (FUV) and by active galactic nuclei (X-ray) in the centers of galaxies. Note, however, that the line intensities (and ratios) presented in this work do not take the, likely complex, kinematics of nuclear gas into account. For example, gas rotating in an accretion disk around an AGN will cause the line widths of, say, HCN, HNC and $\mathrm{HCO}^{+}$transitions to differ, depending on where their chemical abundances peak with depth.

The XDR/AGN contribution will typically be of a much smaller (possibly beam diluted) angular scale than that of a PDR/Starburst. A 10-25\% PDR contribution may already suppress our ability to recognize XDR excitation from $\mathrm{HCN} / \mathrm{HCO}^{+}$ and $\mathrm{HNC} / \mathrm{HCN}$ line ratios. A solution to this can be found in the very high J CO lines (e.g., CO(16-15)), that are excellent indicators of an XDR contribution. These very high rotational lines will be observable with the ESA Herschel (HIFI) space observatory scheduled for launch in the near future; they can be seen in absorption in the near-infrared with Subaru. Currently available (sub)millimeter facilities lack the spatial resolution to separate the PDR/stellar and XDR/AGN contributions in distant active galaxies. Up to now, these components can only be spatially resolved in the Milky Way. However, the resolving power of ALMA will bring this possibility within reach for external galaxy centers as well.

Acknowledgements. We thank Dieter Poelman for making his radiation transfer code beta $3 D$ available to us.

\section{References}

Aalto, S. 2005, in IAU Symp., ed. C. L. Dariusz, A. B. Geoffrey, \& H. Eric, 261 Baan, W. A., \& Klöckner, H.-R. 2005, Ap\&SS, 295, 263

Bergin, E. A., Melnick, G. J., Stauffer, J. R., et al. 2000, ApJ, 539, L129

Curran, S. J., Aalto, S., \& Booth, R. S. 2000, A\&AS, 141, 193

Garnett, D. R., Shields, G. A., Peimbert, M., et al. 1999, ApJ, 513, 168

Garrett, M. A., Muxlow, T. W. B., Garrington, S. T., et al. 2001, A\&A, 366, L5

Hüttemeister, S., \& Aalto, S. 2001, in The Central Kiloparsec of Starbursts and AGN: The La Palma Connection, ed. J. H. Knapen, J. E. Beckman, I. Shlosman, \& T. J. Mahoney, ASP Conf. Ser., 249, 619

Israel, F. P. 2005, Ap\&SS, 295, 171

Israel, F. P., \& Baas, F. 2002, A\&A, 383, 82

Kaufman, M. J., Wolfire, M. G., Hollenbach, D. J., \& Luhman, M. L. 1999, ApJ, 527,795

Kaufman, M. J., Wolfire, M. G., \& Hollenbach, D. J. 2006, ApJ, 644, 283

Klöckner, H.-R., Baan, W. A., \& Garrett, M. A. 2003, Nature, 421, 821

Kobulnicky, H. A., \& Skillman, E. D. 1998, ApJ, 497, 601

Lepp, S., \& Dalgarno, A. 1996, A\&A, 306, L21

Maloney, P. R. 1999, Ap\&SS, 266, 207

Maloney, P. R., Hollenbach, D. J., \& Tielens, A. G. G. M. 1996, ApJ, 466, 561

Meijerink, R., \& Spaans, M. 2005, A\&A, 436, 397

Meijerink, R., Spaans, M., \& Israel, F. P. 2006, ArXiv Astrophysics e-prints

Ott, J., Weiss, A., Henkel, C., \& Walter, F. 2005, ApJ, 629, 767

Poelman, D. R., \& Spaans, M. 2005, A\&A, 440, 559

Poelman, D. R., \& Spaans, M. 2006, ArXiv Astrophysics e-prints

Röllig, M., Abel, N., Bell, T., et al. 2006, in preparation

Röllig, M., Ossenkopf, V., Jeyakumar, S., Stutzki, J., \& Sternberg, A. 2006, arXiv:Astrophysics e-prints

Sanders, D. B., \& Mirabel, I. F. 1996, ARA\&A, 34, 749

Schöier, F. L., van der Tak, F. F. S., van Dishoeck, E. F., \& Black, J. H. 2005, A\&A, 432, 369

Silk, J. 2005, MNRAS, 364, 1337

Spaans, M., \& van Dishoeck, E. F. 2001, ApJ, 548, L217

Spoon, H. W. W., Keane, J. V., Tielens, A. G. G. M., Lutz, D., \& Moorwood, A. F. M. 2001, A\&A, 365, L353

Spoon, H. W. W., Moorwood, A. F. M., Pontoppidan, K. M., et al. 2003, A\&A, 402, 499

Spoon, H. W. W., Keane, J. V., Cami, J., et al. 2005, in IAU Symp., ed. C. L. Dariusz, A. B. Geoffrey, \& H. Eric, 281

Stäuber, P., Doty, S. D., van Dishoeck, E. F., \& Benz, A. O. 2005, A\&A, 440, 949

Vila-Costas, M. B., \& Edmunds, M. G. 1992, MNRAS, 259, 121

Wolfire, M. G., Hollenbach, D., \& Tielens, A. G. G. M. 1989, ApJ, 344, 770

Wolfire, M. G., Tielens, A. G. G. M., \& Hollenbach, D. 1990, ApJ, 358, 116

Zaritsky, D., Kennicutt, R. C., \& Huchra, J. P. 1994, ApJ, 420, 87 
R. Meijerink et al.: Diagnostics of irradiated dense gas in galaxy nuclei. II., Online Material p 1

\section{Online Material}


R. Meijerink et al.: Diagnostics of irradiated dense gas in galaxy nuclei. II., Online Material p 2 Appendix A: On-line figures 
R. Meijerink et al.: Diagnostics of irradiated dense gas in galaxy nuclei. II., Online Material p 3
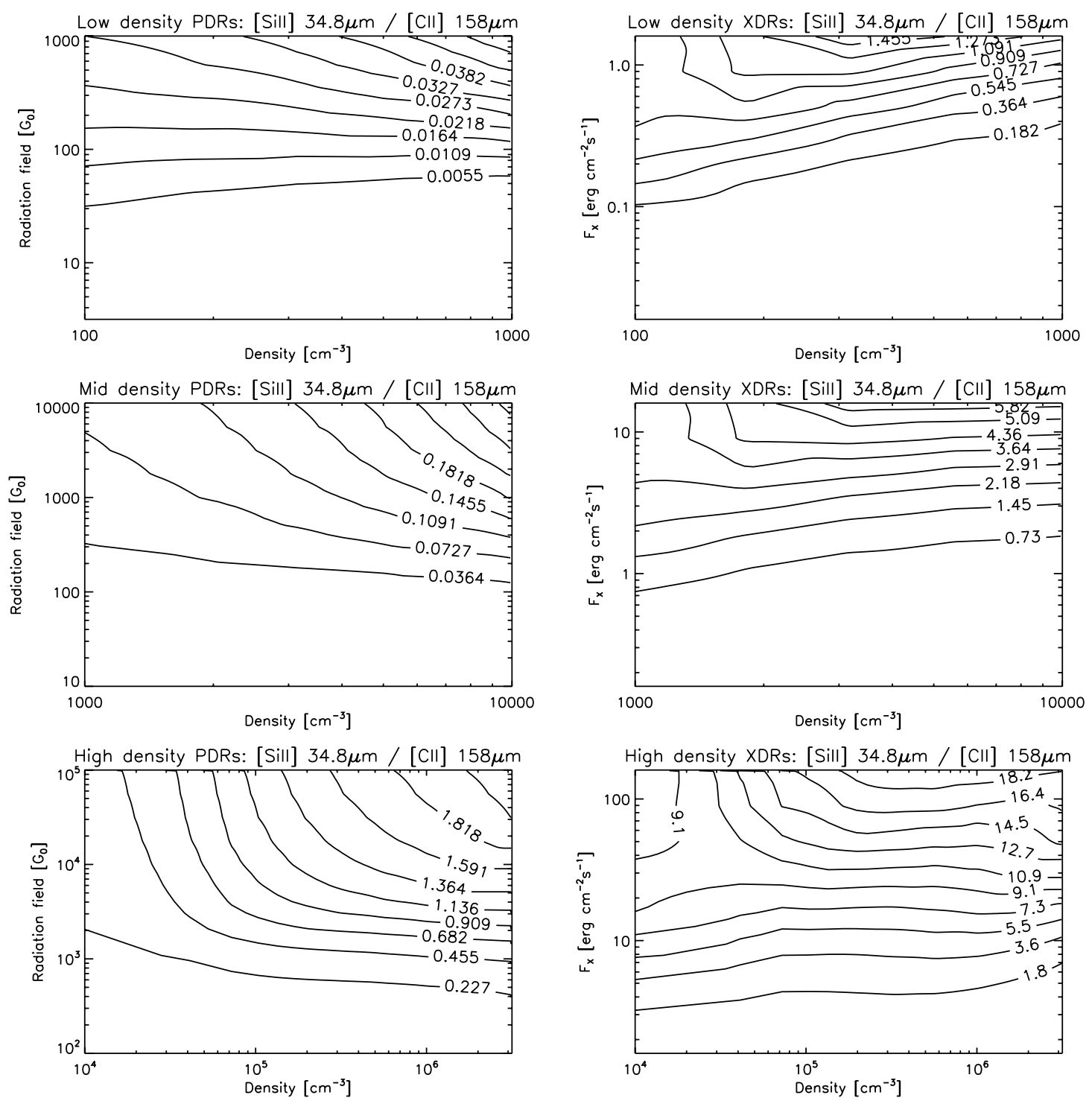

Fig. A.1. [SiII] $34.8 \mu \mathrm{m} /[\mathrm{CII}] 158 \mu \mathrm{m}$ ratio for PDR (left) and XDR (right) models. 
R. Meijerink et al.: Diagnostics of irradiated dense gas in galaxy nuclei. II., Online Material p 4
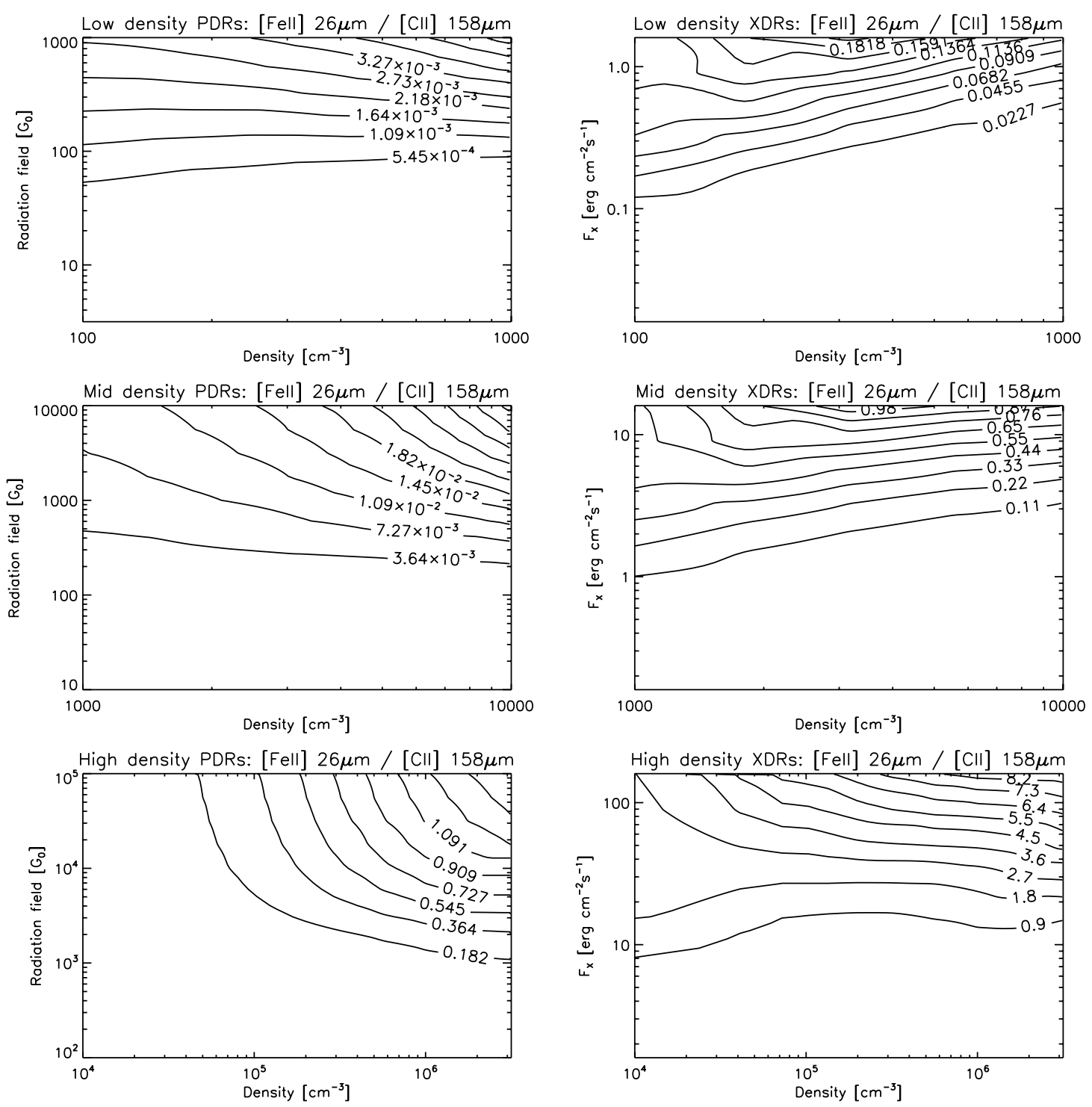

Fig. A.2. [FeII] $26 \mu \mathrm{m} /[\mathrm{CII}] 158 \mu \mathrm{m}$ ratio for PDR (left) and XDR (right) models. 
R. Meijerink et al.: Diagnostics of irradiated dense gas in galaxy nuclei. II., Online Material p 5
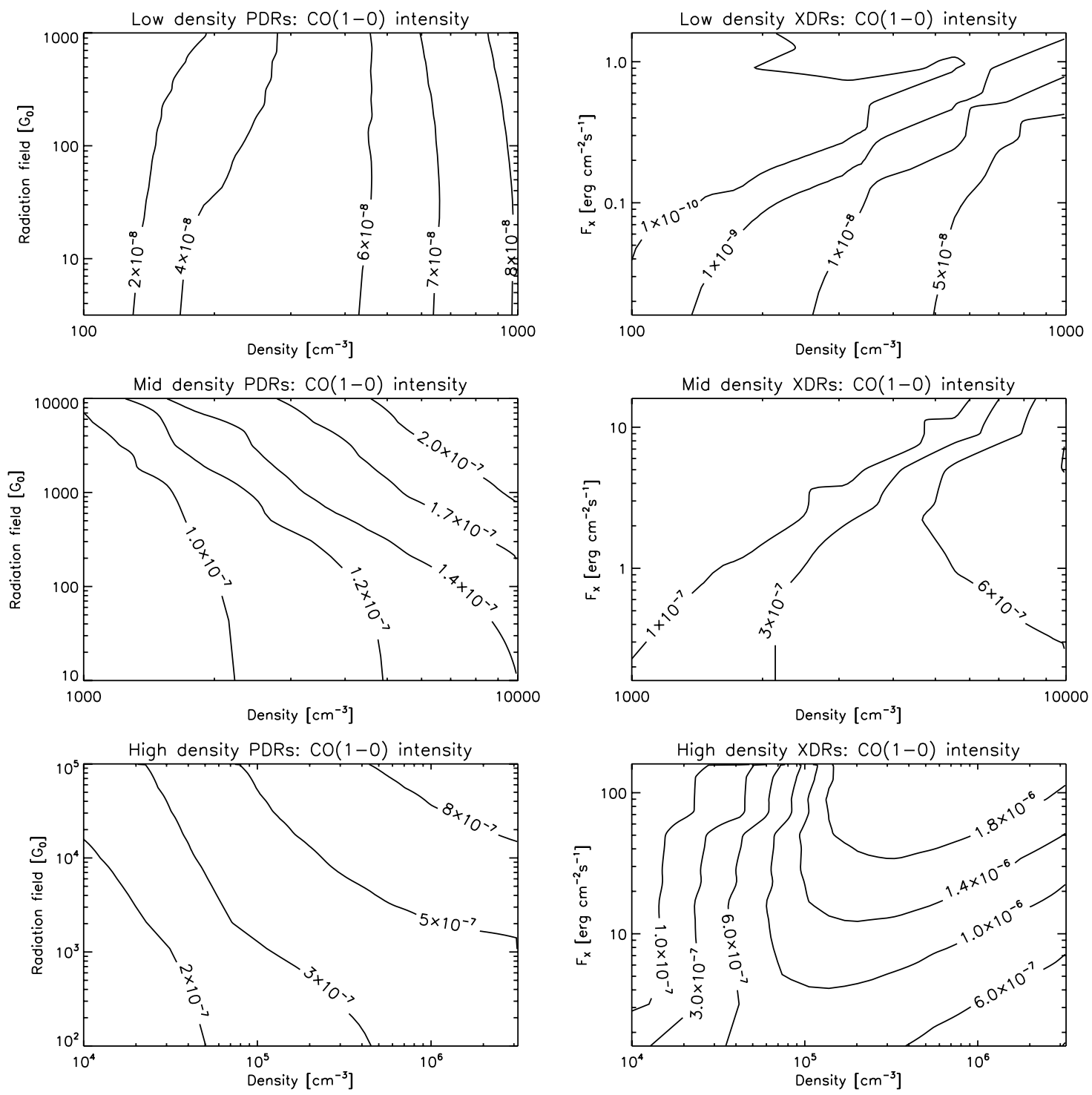

Fig. A.3. CO(1-0) intensity for PDR (left) and XDR (right) models. 
R. Meijerink et al.: Diagnostics of irradiated dense gas in galaxy nuclei. II., Online Material p 6
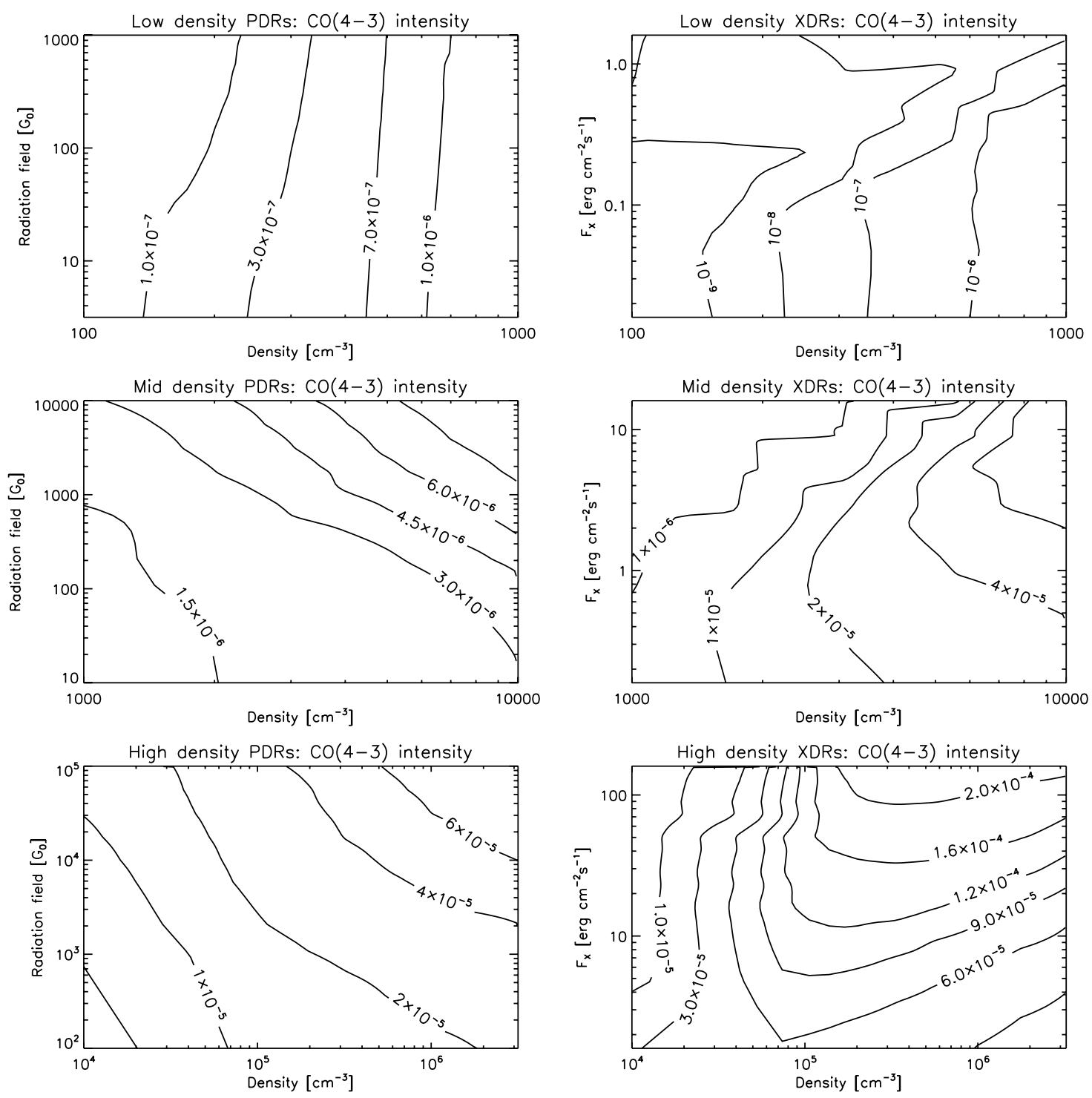

Fig. A.4. CO(4-3) intensity for PDR (left) and XDR (right) models. 
R. Meijerink et al.: Diagnostics of irradiated dense gas in galaxy nuclei. II., Online Material $p 7$
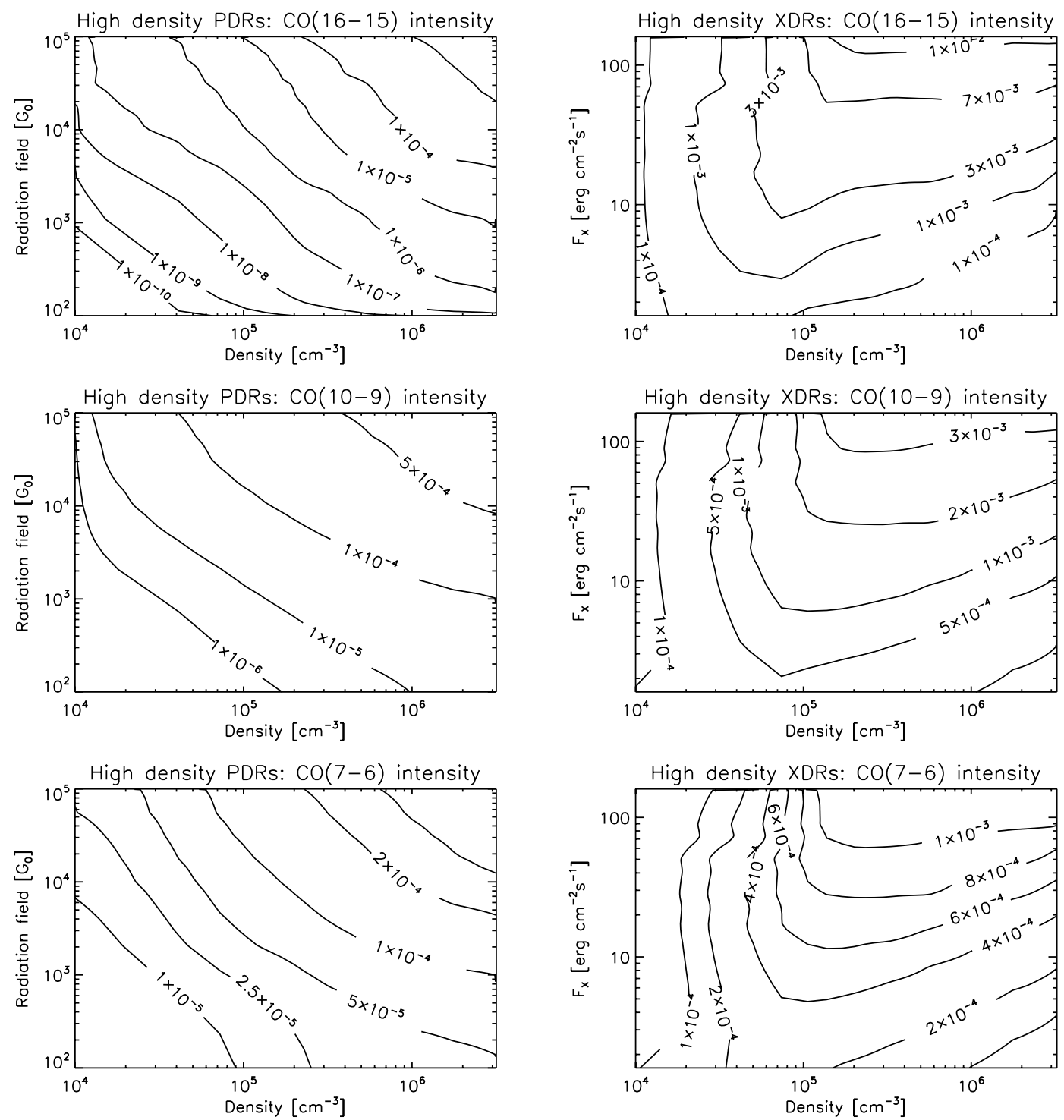

Fig. A.5. $\mathrm{CO}(16-15), \mathrm{CO}(10-9)$, and $\mathrm{CO}(7-6)$ intensity for PDR (left) and XDR (right) models. 
R. Meijerink et al.: Diagnostics of irradiated dense gas in galaxy nuclei. II., Online Material $p 8$
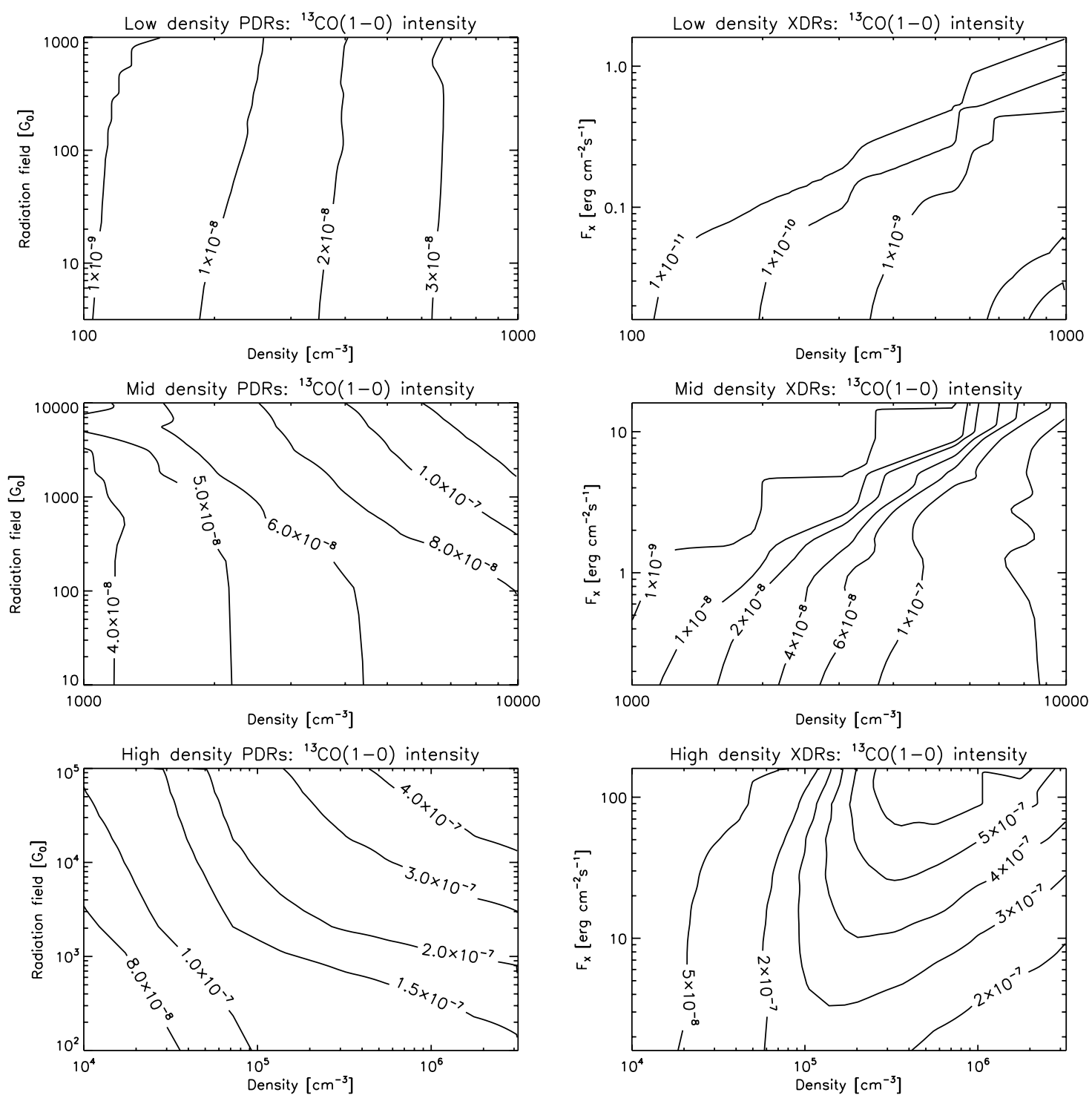

Fig. A.6. ${ }^{13} \mathrm{CO}(1-0)$ intensity for PDR (left) and XDR (right) models. 
R. Meijerink et al.: Diagnostics of irradiated dense gas in galaxy nuclei. II., Online Material $p 9$
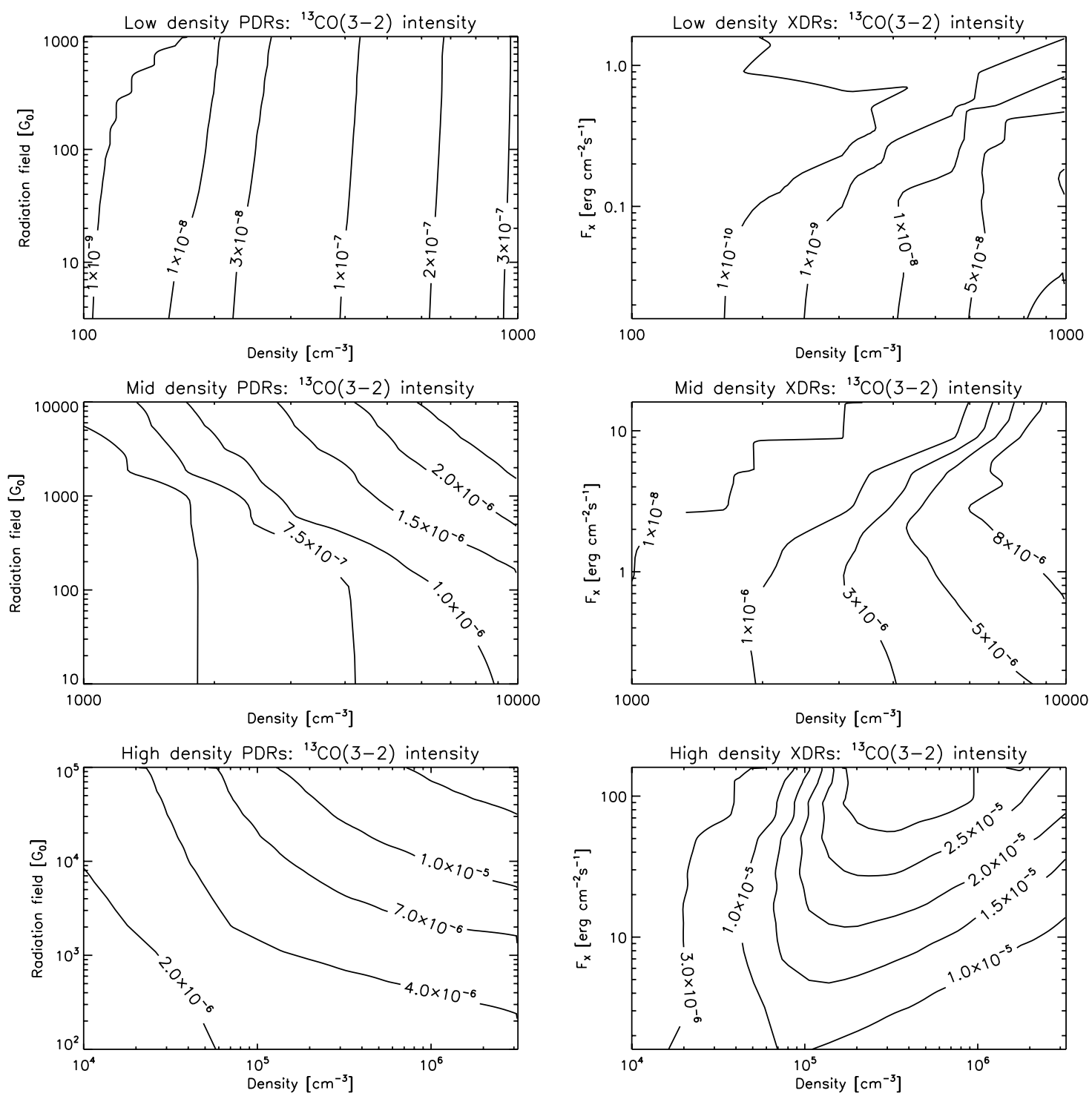

Fig. A.7. ${ }^{13} \mathrm{CO}(3-2)$ intensity for PDR (left) and XDR (right) models. 
R. Meijerink et al.: Diagnostics of irradiated dense gas in galaxy nuclei. II., Online Material p 10
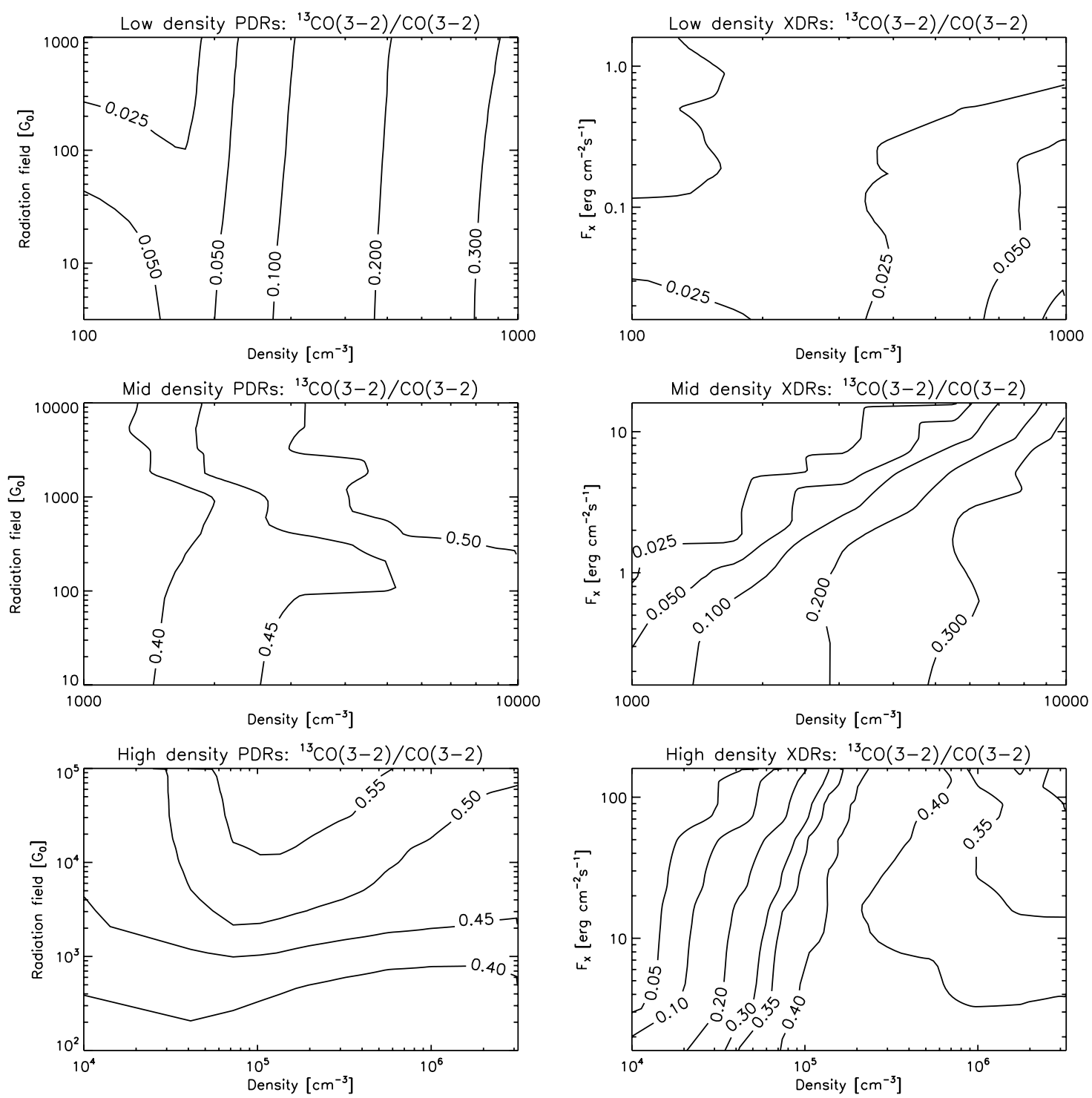

Fig. A.8. ${ }^{13} \mathrm{CO}(3-2) / \mathrm{CO}(3-2)$ ratio for PDR (left) and XDR (right) models. 
R. Meijerink et al.: Diagnostics of irradiated dense gas in galaxy nuclei. II., Online Material p 11
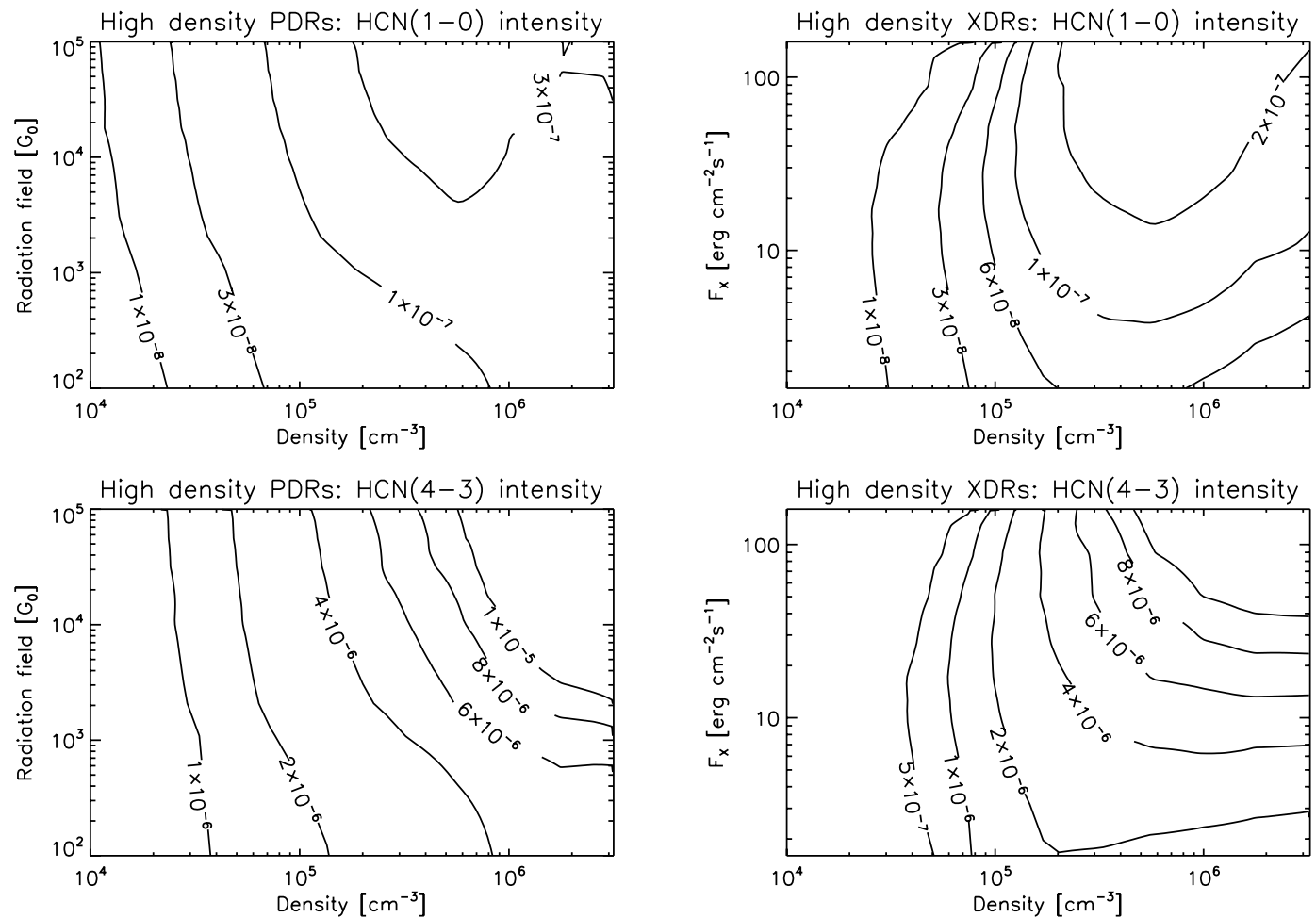

Fig. A.9. $\mathrm{HCN}(1-0)$ and $\mathrm{HCN}(4-3)$ intensity for PDR (left) and XDR (right) models.
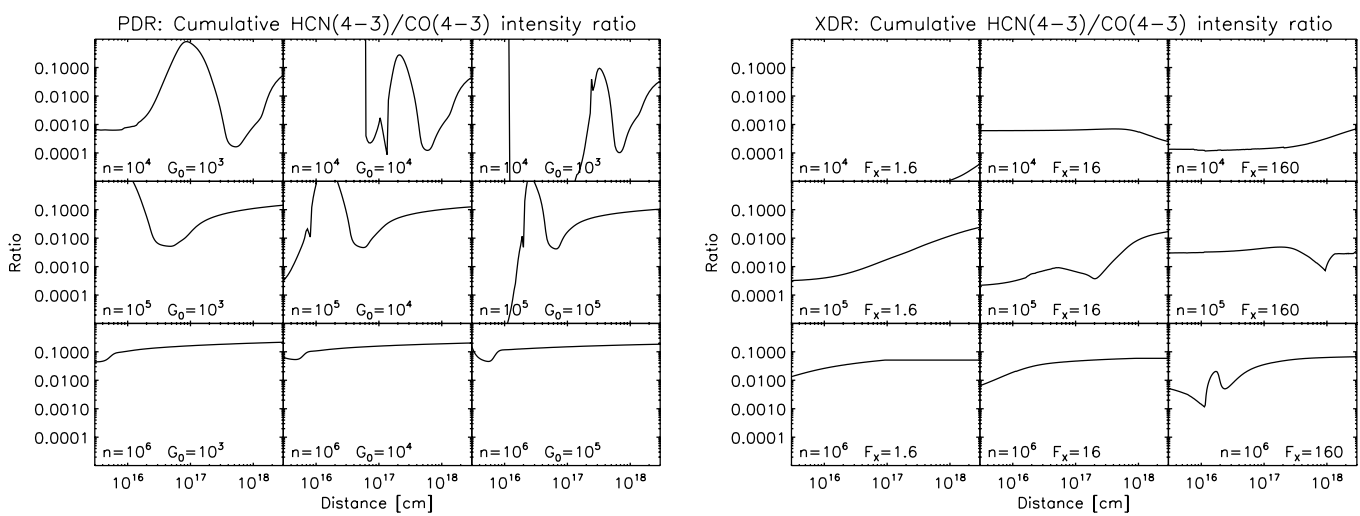

Fig. A.10. Cumulative $\mathrm{HCN}(4-3) / \mathrm{CO}(4-3)$ line intensity ratios for PDR (left) and XDR (right). 
R. Meijerink et al.: Diagnostics of irradiated dense gas in galaxy nuclei. II., Online Material p 12
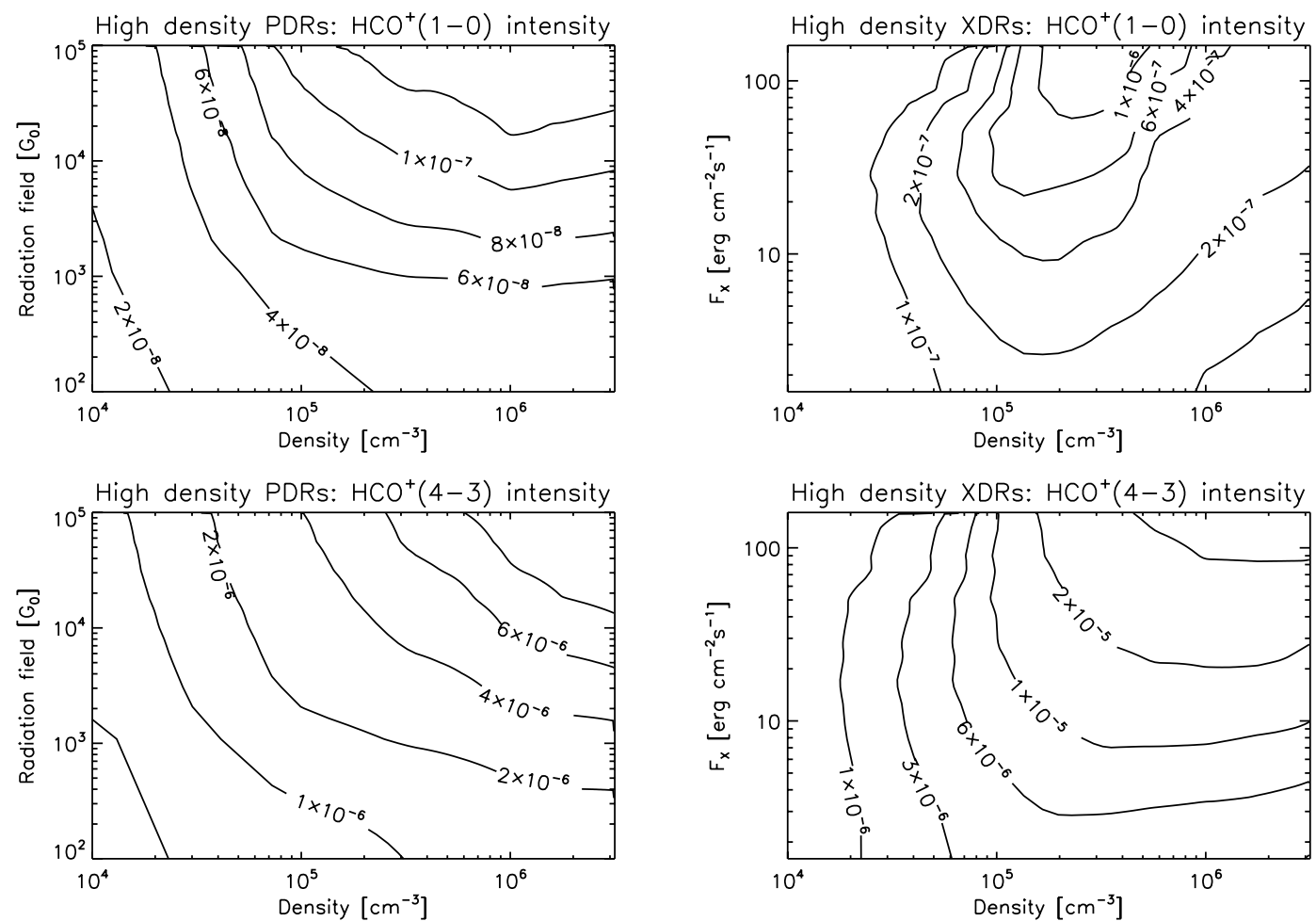

Fig. A.11. $\mathrm{HCO}^{+}(1-0)$ and $\mathrm{HCO}^{+}(4-3)$ intensity for PDR (left) and XDR (right) models.
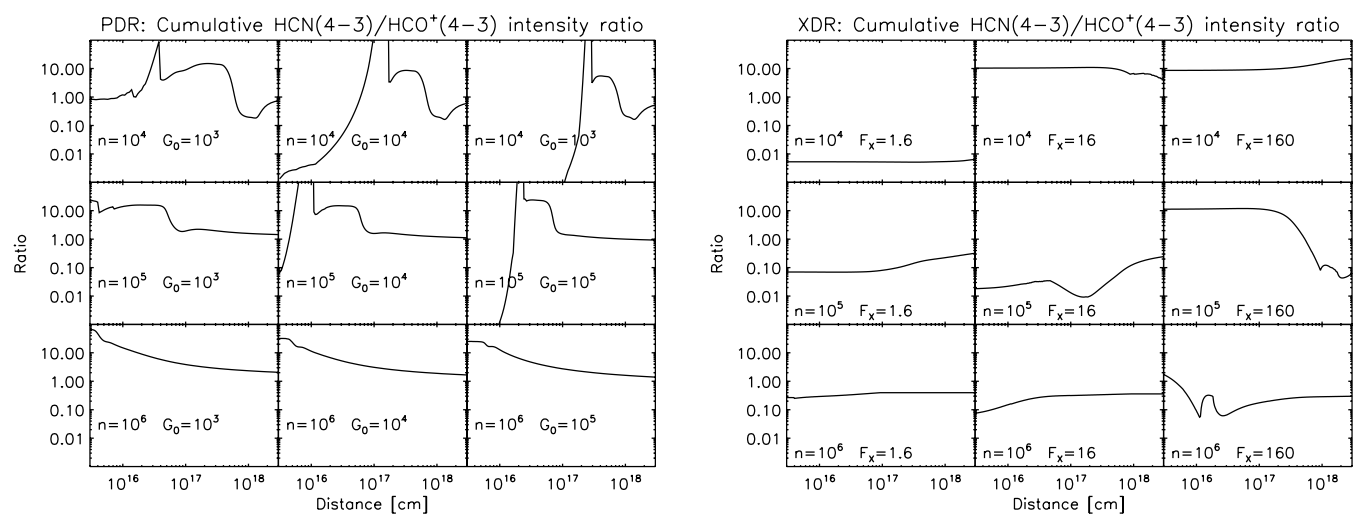

Fig. A.12. Cumulative $\mathrm{HCN}(4-3) / \mathrm{HCO}^{+}(4-3)$ line intensity ratios for PDR (left) and XDR (right).
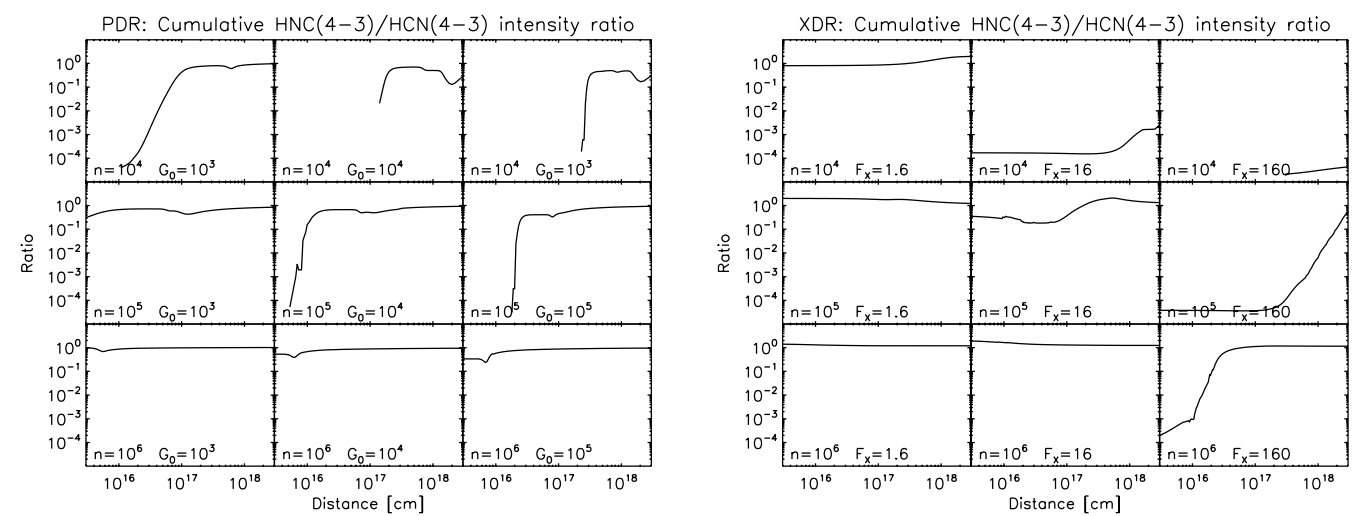

Fig. A.13. Cumulative $\mathrm{HNC}(4-3) / \mathrm{HCN}(4-3)$ line intensity ratios for PDR (left) and XDR (right). 
R. Meijerink et al.: Diagnostics of irradiated dense gas in galaxy nuclei. II., Online Material $p 13$
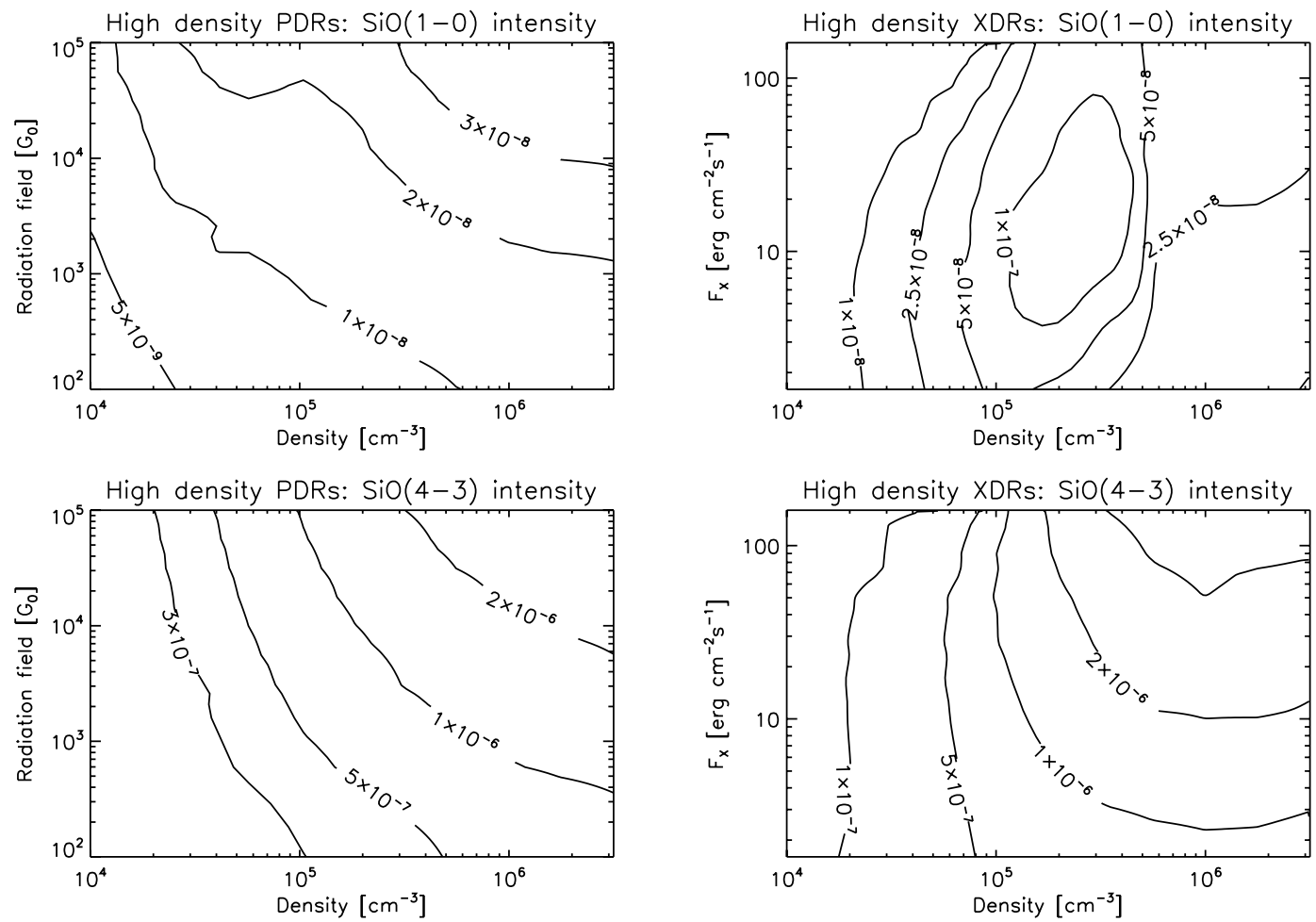

Fig. A.14. $\mathrm{SiO}(1-0)$ and $\mathrm{SiO}(4-3)$ intensity for PDR (left) and XDR (right) models.
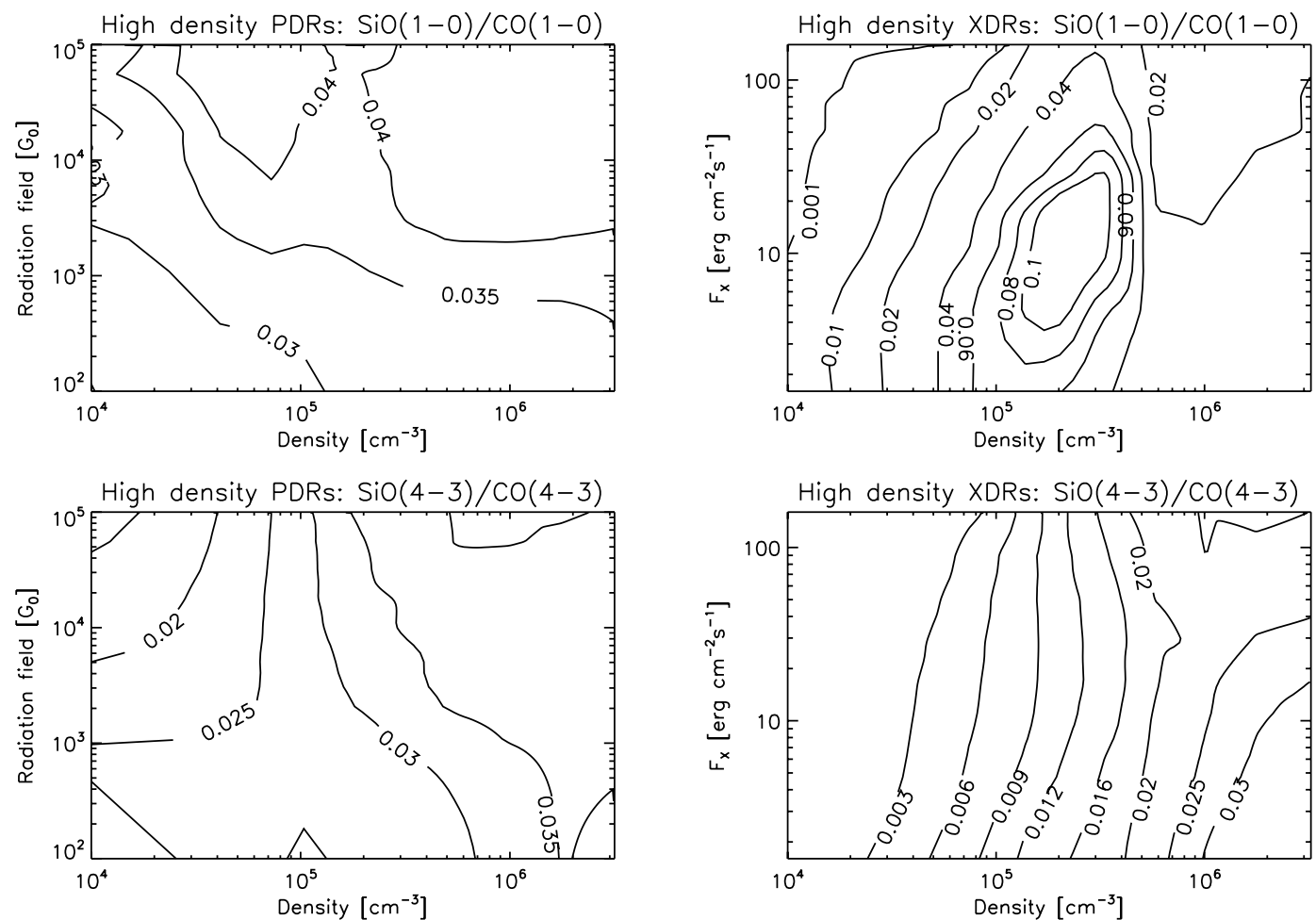

Fig. A.15. $\mathrm{SiO}(1-0) / \mathrm{CO}$ and $\mathrm{SiO}(4-3) / \mathrm{CO}(4-3)$ ratios for PDR (left) and XDR (right) models. 
R. Meijerink et al.: Diagnostics of irradiated dense gas in galaxy nuclei. II., Online Material p 14
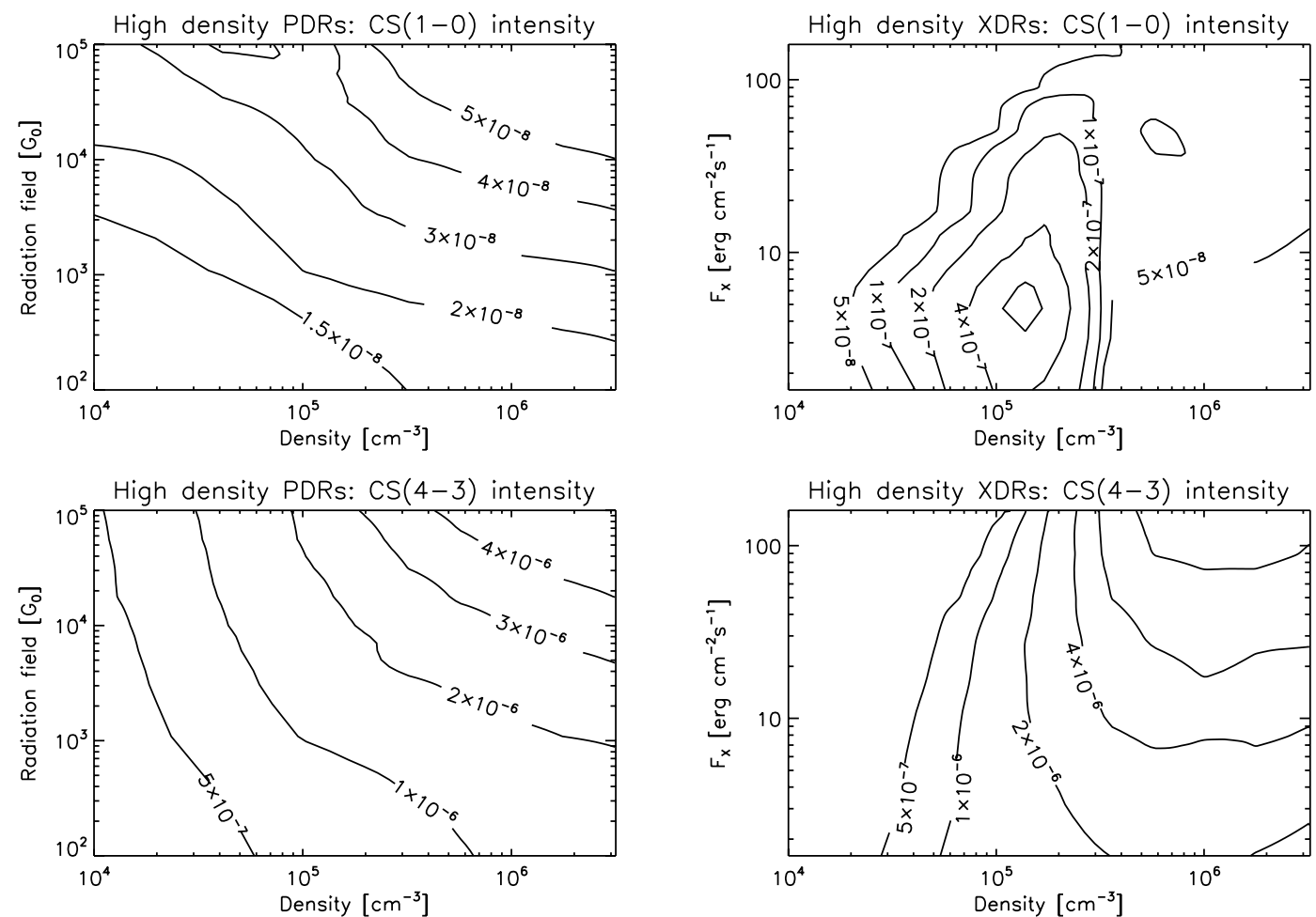

Fig. A.16. $\mathrm{SiO}(1-0)$ and $\mathrm{SiO}(4-3)$ intensity for PDR (left) and XDR (right) models.
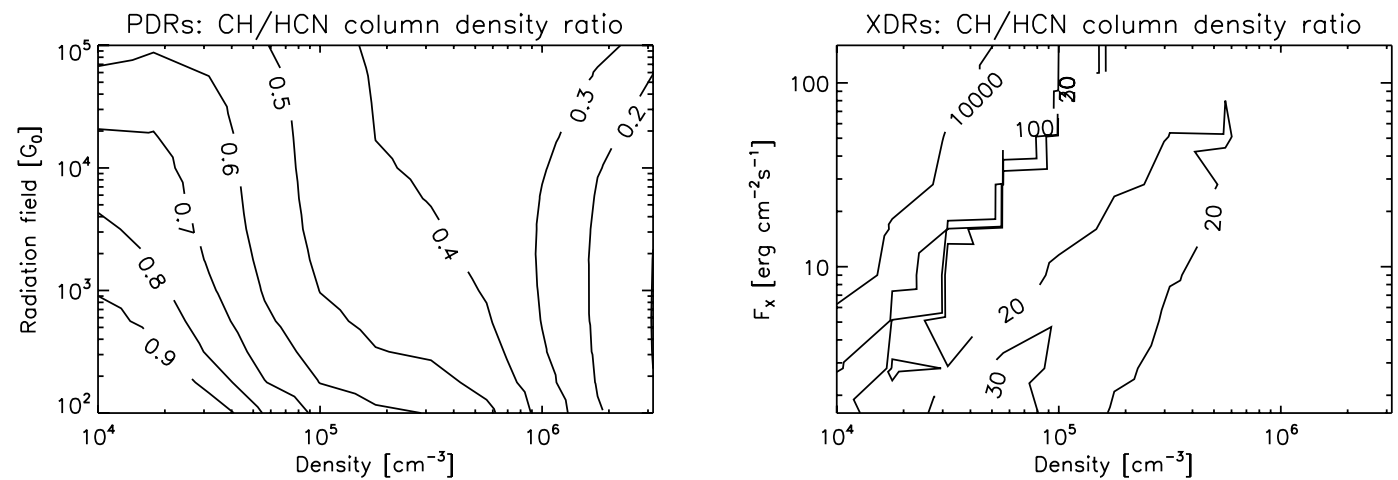

Fig. A.17. $\mathrm{CH} / \mathrm{HCN}$ column density ratios for PDR (left) and XDR (right) models.
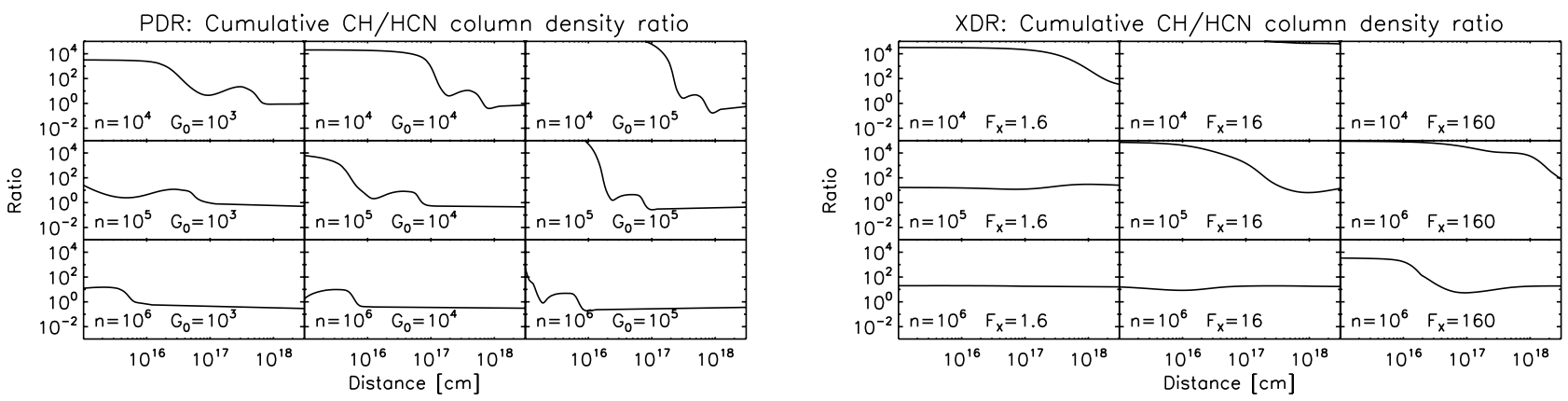

Fig. A.18. Cumulative $\mathrm{CH} / \mathrm{HCN}$ column density ratios for PDR (left) and XDR (right) models. 
R. Meijerink et al.: Diagnostics of irradiated dense gas in galaxy nuclei. II., Online Material p 15
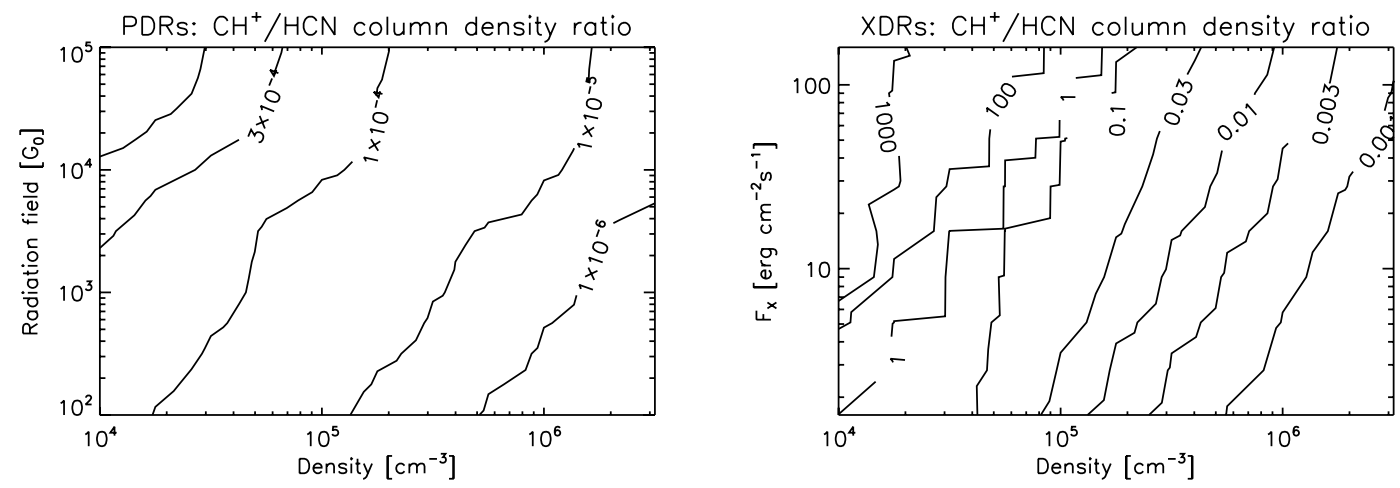

Fig. A.19. $\mathrm{CH}^{+} / \mathrm{HCN}$ column density ratios for PDR (left) and XDR (right) models.
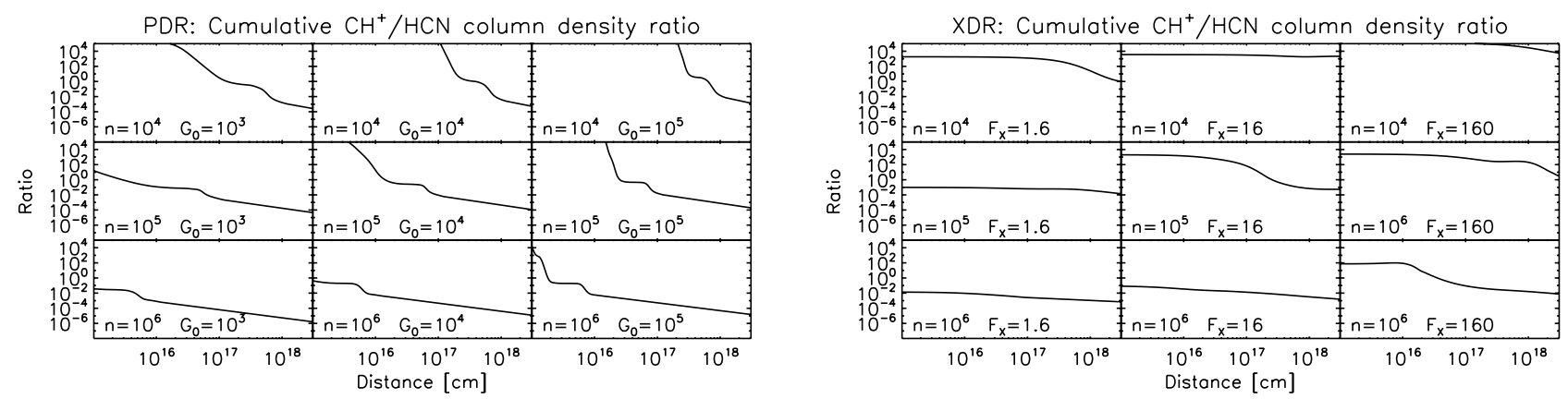

Fig. A.20. Cumulative $\mathrm{CH}^{+} / \mathrm{HCN}$ column density ratios for PDR (left) and XDR (right) models.
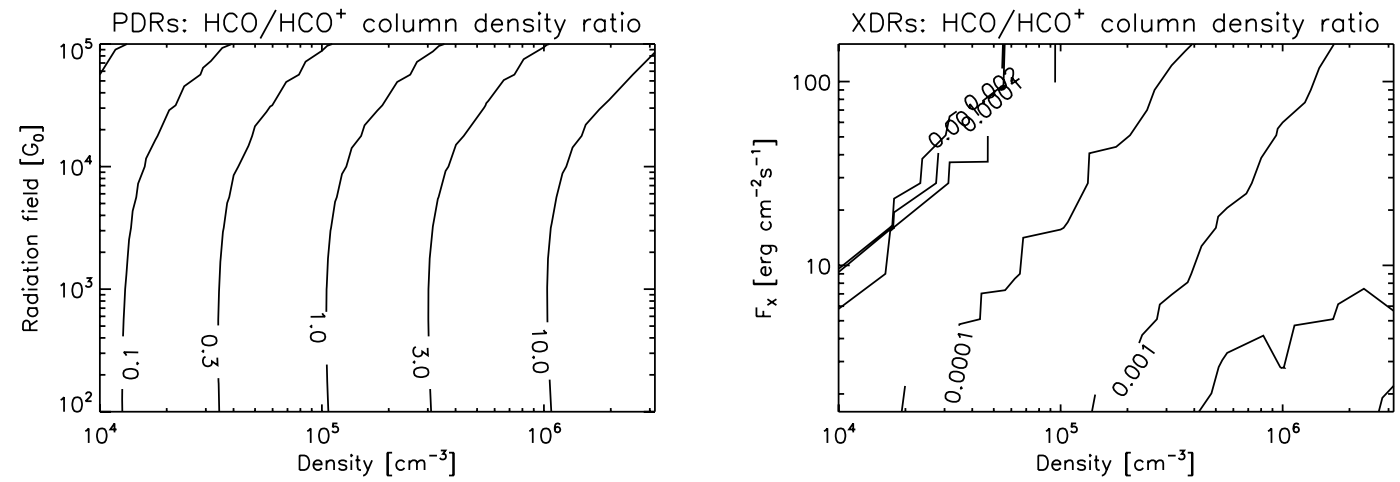

Fig. A.21. $\mathrm{HOC} / \mathrm{HCO}^{+}$column density ratios for PDR (left) and XDR (right) models.
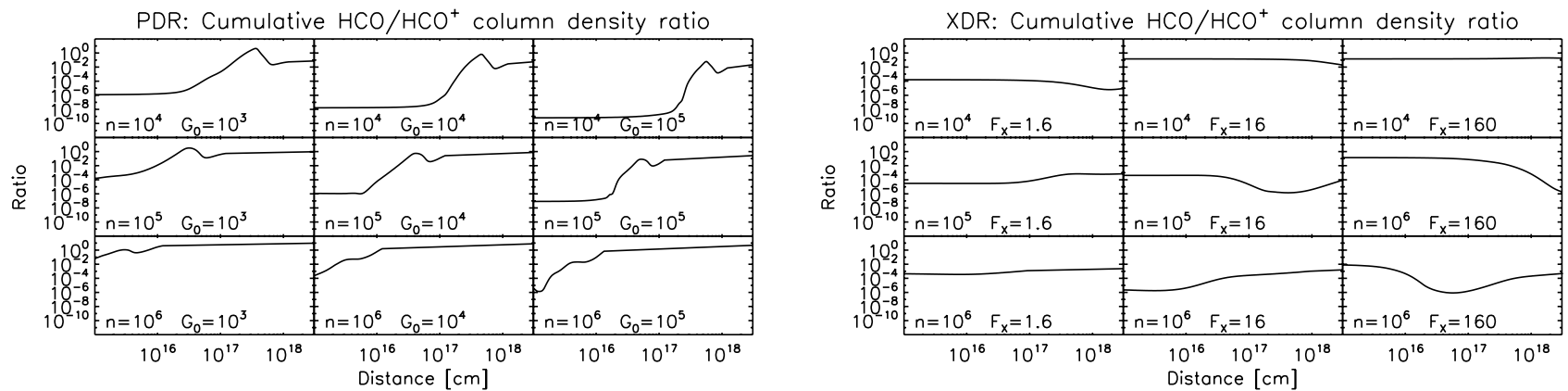

Fig. A.22. Cumulative $\mathrm{HCO} / \mathrm{HCO}^{+}$column density ratios for PDR (left) and XDR (right) models. 
R. Meijerink et al.: Diagnostics of irradiated dense gas in galaxy nuclei. II., Online Material p 16
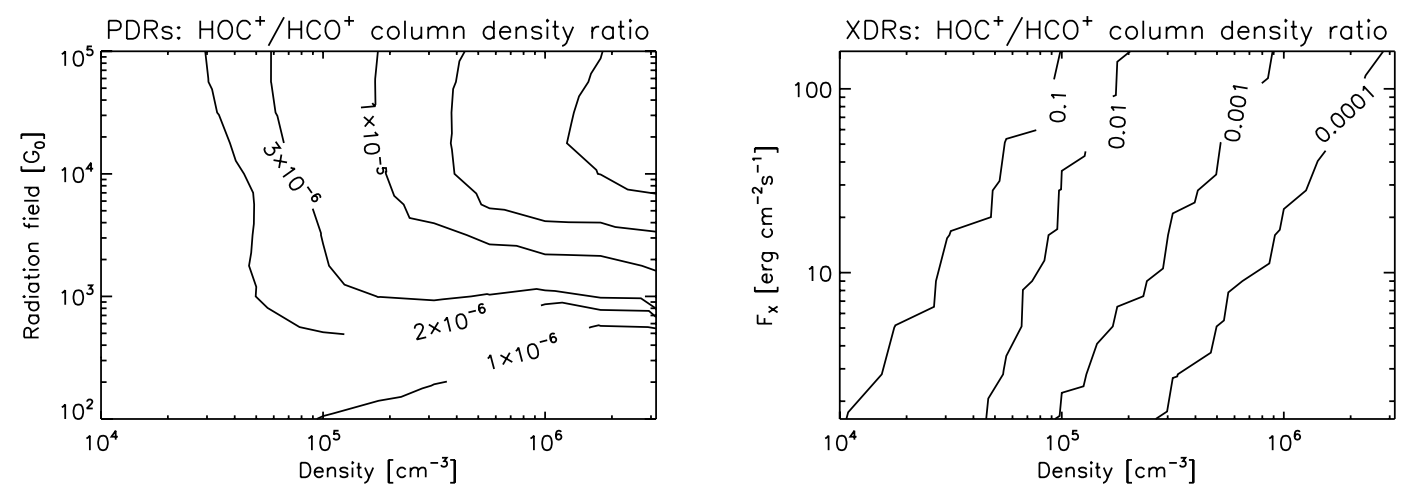

Fig. A.23. $\mathrm{HOC}^{+} / \mathrm{HCO}^{+}$column density ratios for PDR (left) and XDR (right) models.
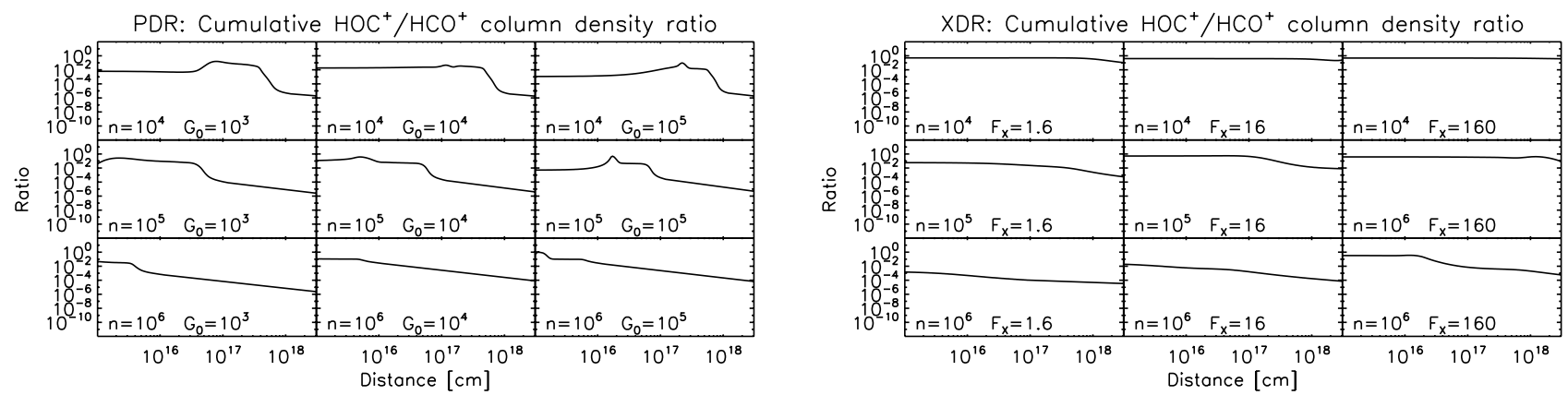

Fig. A.24. Cumulative $\mathrm{HOC}^{+} / \mathrm{HCO}^{+}$column density ratios for PDR (left) and XDR (right) models.
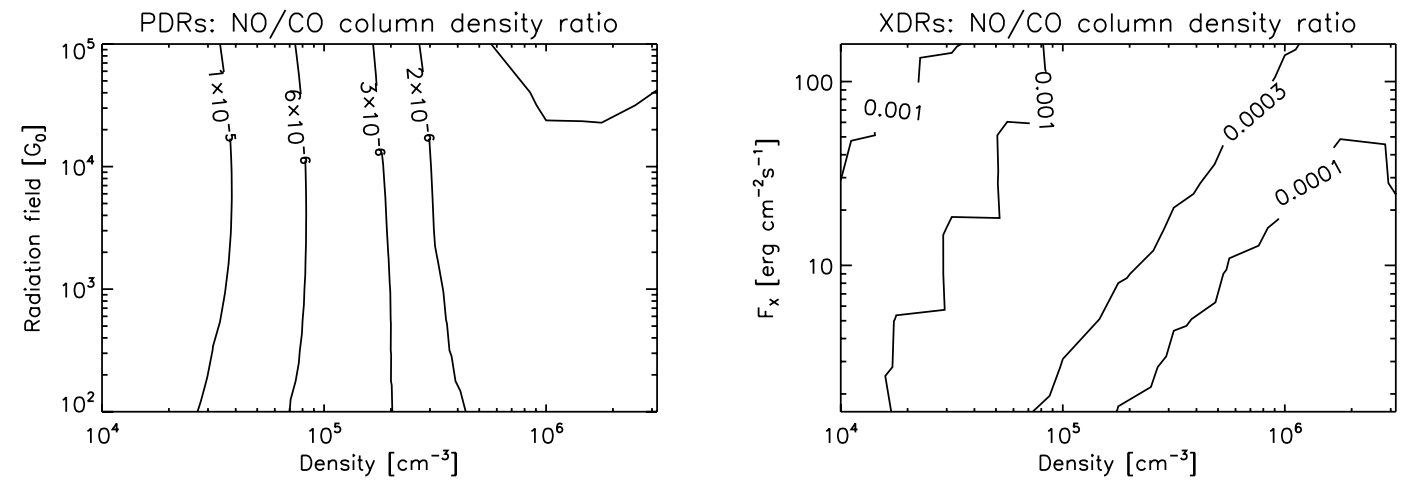

Fig. A.25. NO/CO column density ratios for PDR (left) and XDR (right) models. 
R. Meijerink et al.: Diagnostics of irradiated dense gas in galaxy nuclei. II., Online Material p 17
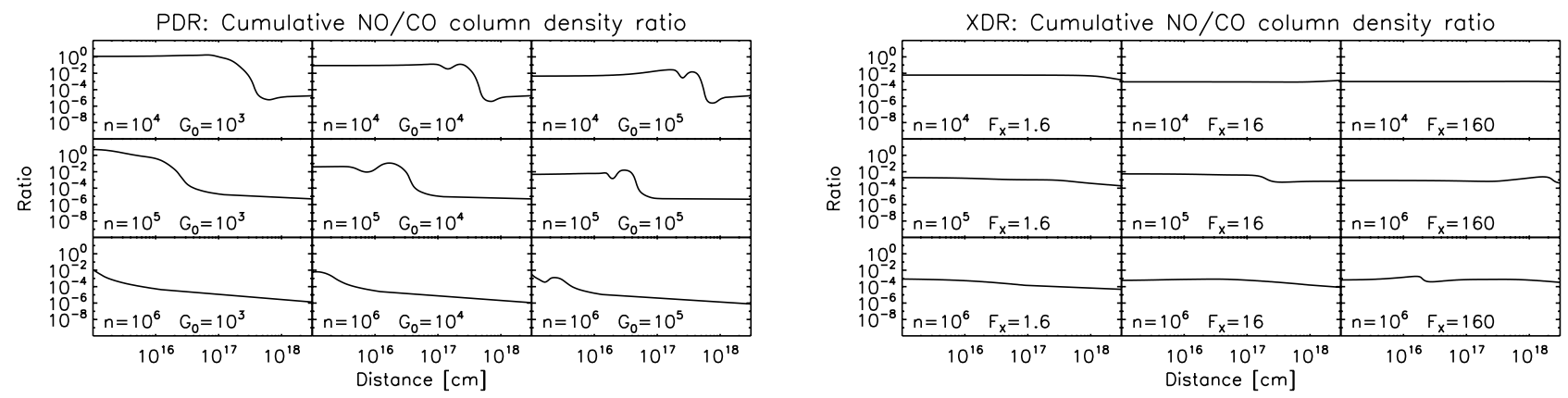

Fig. A.26. Cumulative NO/CO column density ratios for PDR (left) and XDR (right) models.
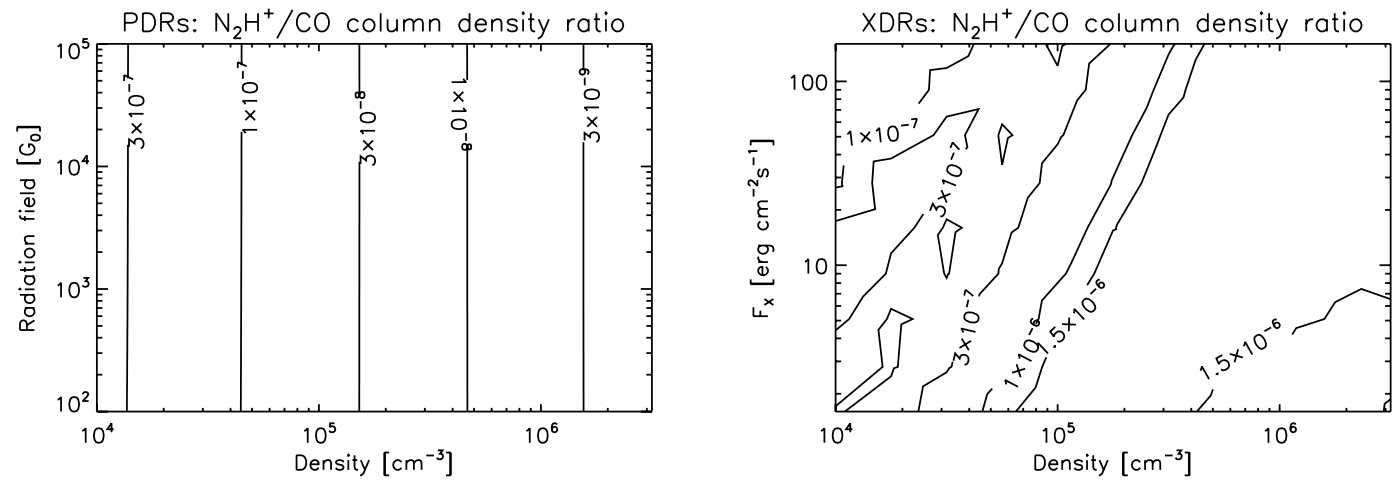

Fig. A.27. $\mathrm{N}_{2} \mathrm{H}^{+} / \mathrm{CO}$ column density ratios for PDR (left) and XDR (right) models.
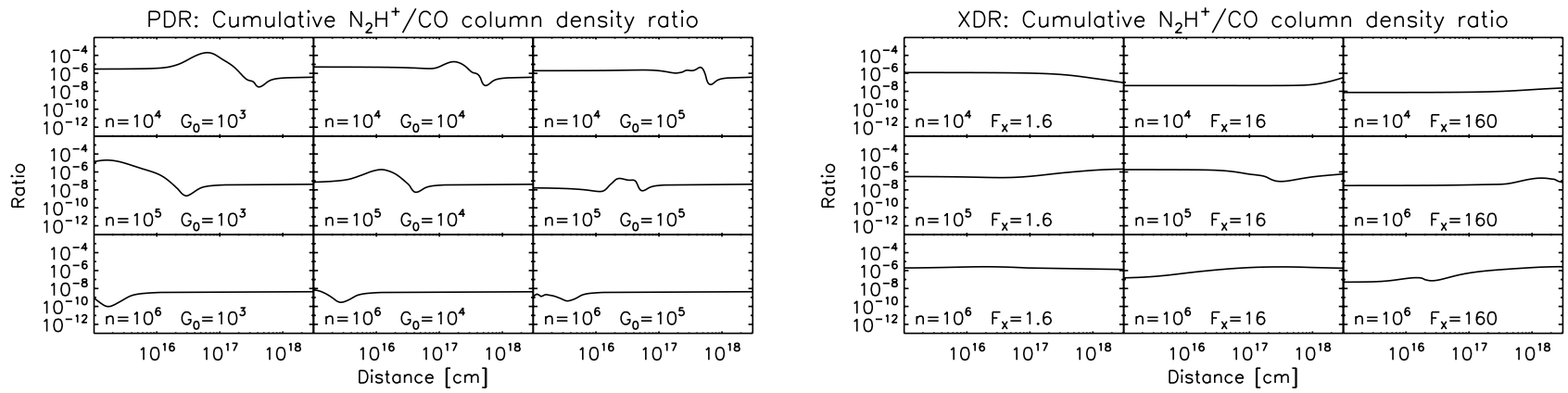

Fig. A.28. Cumulative $\mathrm{N}_{2} \mathrm{H}^{+} / \mathrm{CO}$ column density ratios for PDR (left) and XDR (right) models. 